\title{
MULTI-CHANNEL GROUND-PENETRATING RADAR FOR THE CONTINUOUS QUANTIFICATION OF SNOW AND FIRN DENSITY, DEPTH, AND ACCUMULATION
}

\author{
by \\ Tate Meehan
}

\author{
A thesis \\ submitted in partial fulfillment \\ of the requirements for the degree of \\ Master of Science in Geophysics \\ Boise State University
}

May 2018 
(c) 2018

Tate Meehan

ALL RIGHTS RESERVED 
BOISE STATE UNIVERSITY GRADUATE COLLEGE

\title{
DEFENSE COMMITTEE AND FINAL READING APPROVALS
}

\author{
of the thesis submitted by
}

\author{
Tate Meehan
}

Thesis Title: Multi-channel Ground-penetrating Radar for the Continuous Quantification of Snow and Firn Density, Depth, and Accumulation

Date of Final Oral Examination: 16 February 2018

The following individuals read and discussed the dissertation submitted by student Tate Meehan, and they evaluated the students presentation and response to questions during the final oral examination. They found that the student passed the final oral examination.

John Bradford Ph.D. Chair, Supervisory Committee

H.P. Marshall Ph.D. Member, Supervisory Committee

Dylan Mikesell Ph.D. Member, Supervisory Committee

The final reading approval of the thesis was granted by John Bradford Ph.D., Chair of the Supervisory Committee. The thesis was approved by the Graduate College. 


\title{
DEDICATION
}

\author{
fluff kitten \\ in the snow hidden \\ a tiny bird in \\ the place of a million \\ black bananas and constant sunrise \\ wavelengths sensitive to grainsize \\ hawaiian shirts, chocolate cake \\ geophysics will and can take \\ people places ill never go \\ like my mate tate on the snow \\ - Daniel Planas
}

Dedicated to all people who supported the GreenTrACS campaign, spiritually, logistically, or otherwise.

Thank you. 


\section{ACKNOWLEDGMENTS}

When I was accepted into the Master of Geophysics program at Boise State University, I received a casual email from my future adviser. Dr. John Bradford, asked if I was interested in participating in a research campaign to study Greenland firn. While reading his email I had two thoughts in mind: "How cold is it in Greenland?", and "What is firn?". At this time, three years ago, I had little confidence about pursuing a career in geophysics. After graduating with my Bachelor's at the tail end of the oil price turn down, in Texas, the prospect for a career in exploration geophysics was scarce. After countless job applications had been rejected or unreviewed, I leaped at the opportunity to explore Greenland.

Fast-forward to May, 2016. I was being off-loaded from a United States Air Force LC-130 onto a barren, flat, white expanse with several hundred thousand dollars of radar and ice drilling equipment with research scientists, whom I had just met in the weeks prior. I learned heaps from this group of people. I want to express sincere thanks to the GreenTrACS members - Dr. H.P. Marshall, Dr. Bob Hawley, Dr. Erich Osterberg, Thomas Overly, Gabe Lewis, Karina Graeter, and not least for our safety and entertainment Forrest McCarthy - for the months of support and teamwork throughout the 2016 and 2017 expeditions. This experience has been the most formative of my early career in geophysics. Of course none of the science could have been conducted with the support of the CH2M Hill Polar Services crew. GreenTrACS 
expresses our gratitude to all of the support staff and the Air National Guard $109^{\text {th }}$ Airlift Wing for safely transporting us on and off the ice.

Dr. Bradford saw the potential in me and expressed his patience and expertise throughout these three years. He allowed me to approach this multi-channel radar problem creatively. I developed my understanding radar reflection velocimetry from time-zero, through several methods of analysis, until working to the result published within this thesis. I also want to express my gratefulness for my cubicle mate Matt Von Lintig. He and I would motivate our thesis work through many long days, late nights, and forsaken Saturdays.

I am grateful for my family. While I endured the ice sheet spring time, they endured my absence. Thanks to grandad Orville for his humor and enthusiasm supporting our "secret missions". Thanks to my father John and my mother Carole for encouraging me to pursue this experience wholeheartedly. Thanks to my girlfriend Mackenzie for loving me and always feeling one day closer. 


\begin{abstract}
A priority of ice sheet surface mass balance (SMB) prediction is ascertaining the surface density and annual snow accumulation. These forcing data are inputs for firn density models and can be used to inform remotely sensed ice sheet surface processes and to assess Regional Climate Model (RCM) skill. The Greenland Traverse for Accumulation and Climate Studies (GreenTrACS) retrieved 16 shallow firn cores and dug 42 snow pits along the Western percolation zone of the Greenland Ice Sheet (GrIS) during May and June of 2016 and 2017. I deployed and maintained a multi-channel $500 \mathrm{MHz}$ ground-penetrating radar in a multi-offset configuration throughout the two traverse campaigns. The multi-channel radar technique accurately and independently estimates density, depth, and annual snow accumulation - between the firn core and snow pit sites - by horizon velocity analysis of common midpoint radar reflections from the snow and shallow firn. I analyzed a $45 \mathrm{~km}$ section of the traverse in a high accumulation zone, known as the GreenTrACS Core 15 Western Spur. Deviations in surface density up to $\pm 15 \mathrm{~kg} / \mathrm{m}^{3}$ from the transect mean correlate with surface elevation and surface slope angle. Spatial variation in mean annual accumulation of $\sim 0.175 \mathrm{~m}$ w.e. $a^{-1}$ occurs across a trough in the surface topography $\sim 5 \mathrm{~km}$ wide. The reported variability of density and accumulation demonstrates that RCMs must be down-scaled to resolutions within $5 \mathrm{~km}$ to assess subtle yet significant contributions to the GrIS SMB.
\end{abstract}




\section{TABLE OF CONTENTS}

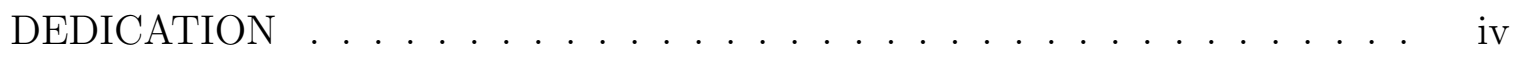

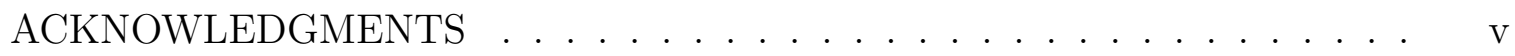

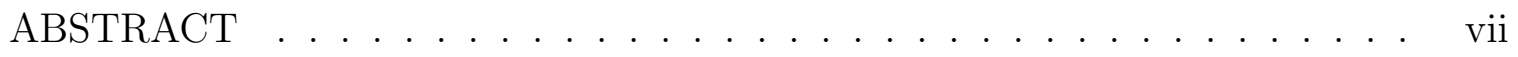

LIST OF TABLES . . . . . . . . . . . . . . . . . . . . xiii

LIST OF FIGURES $\ldots \ldots \ldots \ldots \ldots \ldots \ldots \ldots \ldots \ldots \ldots \ldots$ xiv

LIST OF ABBREVIATIONS . . . . . . . . . . . . . . . xxiii

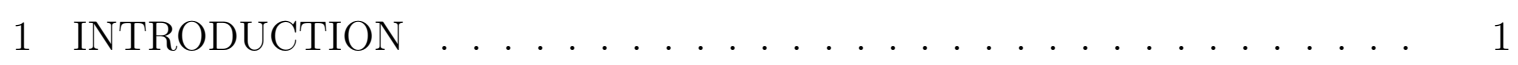

1.0.1 Directory of the Thesis Chapters . . . . . . . . . 2

1.1 Motivation for Advancing the Study of Greenland Mass Balance . . . 4

1.2 Radio Echo Sounding of Ice Sheets _ . . . . . . . . . . 5

1.2.1 RES Studies of Accumulation, Surface Density, and Firn Com-

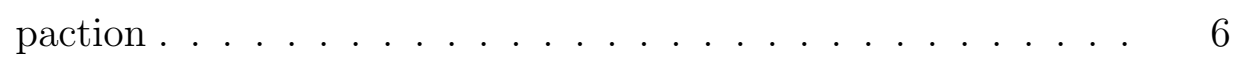

1.2.2 Ground-Penetrating Radar Applications in Polar Firn . . . . . 9

1.2.3 Electromagnetic Velocity Determination of Glaciers: Applica-

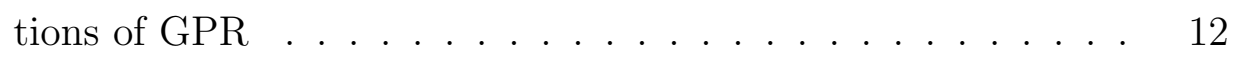

1.3 Implications of this Study for the Wider Scientific Community . . . 16 
2 Methodology: ACQUiSITION, SIGNAL PROCESSING, VELOCITY

ANALYSIS, AND SNOW/FIRN QUANTIFICATION . . . . . . 18

2.1 Traversed Acquisition of Multi-Offset Radar . . . . . . . . . . . 18

2.1.1 Antenna Array Geometry Design . . . . . . . . . . . 20

2.2 Preprocessing . . . . . . . . . . . . . . . . . . . . . . . 24

2.3 Signal Processing . . . . . . . . . . . . . . . . . . . . . . 26

2.4 Horizon Velocity Analysis _. . . . . . . . . . . . . . . . 28

2.4.1 Linear Moveout Analysis of Direct Arrivals . . . . . . . . . 29

2.4 .2 The Air-Coupled Wave . . . . . . . . . . . . . . . 30

2.4.3 Residual Systemic Static Correction . . . . . . . . . . . . . 31

2.4.4 The Surface-Coupled Wave . . . . . . . . . . . . . . . . . 31

2.4.5 Normal Moveout Analysis of Reflected Arrivals . . . . . . . 32

2.4.6 The $x^{2}-t^{2}$ Method and Interval Velocity . . . . . . . . 33

2.4.7 $\mathrm{L}_{\mathbf{1}}$ Minimization for Velocity Regression . . . . . . . . 34

2.4.8 Determining $\mathbf{L}_{\mathbf{1}}$ Solution Convergence . . . . . . . . . 37

2.4.9 Monte Carlo Bootstrapping for Velocity Uncertainty _. . . . 39

2.4 .10 Confidence Intervals $\ldots \ldots \ldots$

2.5 Coupling Electromagnetic Velocity and Polar Snow and Firn . . . . . 41

2.5.1 Complex Refractive Index Method for Estimating Snow and Firn Density . . . . . . . . . . . . . . . . . 41

2.5.2 Jackknife Appraisal of Density and Depth . . . . . . . . 44

2.5.3 An Empirical Model Prediction of Density at Depth . . . . . . 45

2.5.4 Estimating the Snow Accumulation Contribution of GrIS Mass Balance . . . . . . . . . . . . . . 46 
2.5.5 Appraisal of the Accumulation Estimate . . . . . . . . . 47

3 RESULTS: MULTI-CHANNEL RADAR DATA ANALYSIS . . . . . . . 48

3.1 GreenTrACS Core $15 \ldots \ldots \ldots \ldots \ldots$

3.2 Multi-channel Radar Signal Interpretation _. . . . . . . . . 50

3.3 Surface Density, Depth, and Accumulation . . . . . . . . . . 53

3.3.1 Corrections for Instrument and Horizon Depth Biases . . . . 53

3.3.2 Radar Forcing to Extrapolate Density at Depth . . . . . . . 55

3.4 Depth Imaging of Snow and Firn: Stratigraphy, Density, and Age . . 60

3.4.1 Radiostratigraphy-Depth Imaging . . . . . . . . . . . . 60

3.4 .2 Density-Depth Imaging . . . . . . . . . . . . . . . . . . 61

3.4 .3 Age-Depth Imaging . . . . . . . . . . . . . . . . . 62

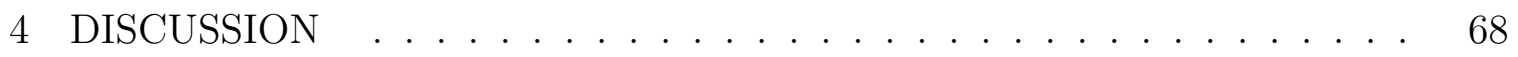

4.1 Known Assertions, Errors, and Remediation of NMO Velocity Analysis 69

4.2 The MxRadar Modeled Approach . . . . . . . . . . . . . . 71

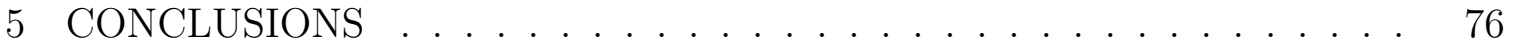

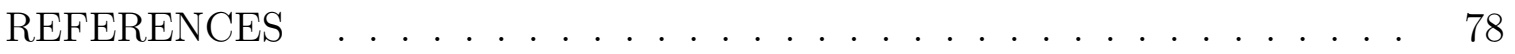

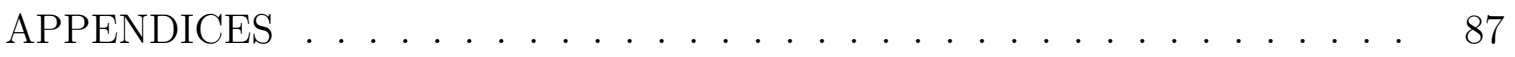

A DATA ACQUISITION ............................ 87

A.1 Pulsed Radio Echo Acquisition _. . . . . . . . . . . . 88

A.2 Frequency Aliasing, Spatial Aliasing, and Stacking . . . . . . . 88 


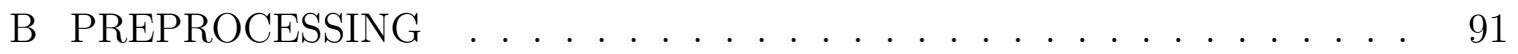

B.1 Installation of Acquisition Geometry . . . . . . . . . . . 92

B.2 De-multiplexing and Data Sorting . . . . . . . . . . . . . 92

B.2.1 Common-Offset Gathers . . . . . . . . . . . . . . . . . 93

B.2.2 Shot Gathers and Common-Midpoint Gathers . . . . . . . 94

B.3 Skipped Trace Removal . . . . . . . . . . . . . . . . . . . . . . . 97

B.3.1 Static Trace Removal . . . . . . . . . . . . . . . . . . . 98

B.4 Trace or Gather Removal . . . . . . . . . . . . . . . . . . . . 102

B.5 Georeferencing Traces . . . . . . . . . . . . . . 102

B.5.1 Static Coordinate Removal . . . . . . . . . . . . . . . . 102

B.5.2 GPS Coordinate Interpolation . . . . . . . . . . . . . 103

B.6 Effect of Skipped Traces and Tow Speed on Midpoint Binning . . . . 103

B.7 Systemic Time-Zero Correction _. . . . . . . . . . . . . 105

C SIGNAL PROCESSING . . . . . . . . . . . . . . . . 106

C.1 De-WOW Filtering . . . . . . . . . . . . . . . . 107

C.1.1 Residual Median Filtering . . . . . . . . . . . . . . 107

C.1.2 Residual Mean Filtering . . . . . . . . . . . . . . . . . 108

C.1.3 Band-Pass Filtering . . . . . . . . . . . . . . . . . . . . . 108

C.1.4 Kill Channels . . . . . . . . . . . . . . . . . . . . . . 110

C.2 Amplitude Recovery Gain . . . . . . . . . . . . . . . . . 111

C.2.1 Recovery of Cable Attenuation _. . . . . . . . . 111

C.2.2 Time-variant Exponential Gain . . . . . . . . . . . . . . 112

C.2.3 Instantaneous AGC Gain . . . . . . . . . . . . . . . . . . 112

C.3 Trace Stacking . . . . . . . . . . . . . . . . . 114 
C.4 Spatial Median Subtraction _. . . . . . . . . . . . . 115

C.5 Spatial Predictive Deconvolution _. . . . . . . . . 116

C.6 Normal Moveout Correction and Depth Conversion . . . . . . . . 120

C.6.1 Stacking Velocity . . . . . . . . . . . . . . 120

C.6.2 Normal Moveout Correction and Stretch _ . . . . . . . . 120

C.6.3 CMP Stacking. . . . . . . . . . . . . . . . . . . . 121

C.6.4 Time to Depth Conversion . . . . . . . . . . . . . . . . 122

D HORIZON VELOCITY ANALYSIS . . . . . . . . . . . . 123

D.1 Semi-Automated Waveform Travel-time Picking . . . . . . . . . 124

D.1.1 Distance Weighted Interpolation of Travel-Time Picks . . . . 125

D.2 Higher-Order NMO Approximation . . . . . . . . . . 126

D.3 Dix's Equations to Determine Interval Velocity . . . . . . . . . 127

D.3.1 Matrix Equation to Determine Interval Velocity . . . . . . . 129

D.3.2 Testing Dix's Equations $\ldots \ldots \ldots \ldots$

D.4 Monte-Carlo Travel-time Simulations to Determine $\mathbf{L}_{\mathbf{1}}$ Convergence

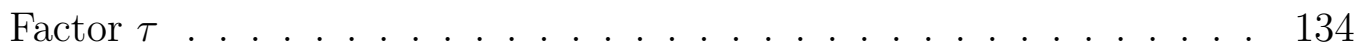

D.5 Critically Refracted Lateral Waves _... . . . . . . . . 139 


\section{LIST OF TABLES}

2.1 The acquisition parameters for the $500 \mathrm{MHz}$ multi-channel system. The two-way recording time window was varied between the two campaigns: 250ns during 2016 and 225ns during 2017. . . . . . . . . . . . 20

2.2 The cycle of the nine channel $500 \mathrm{MHz}$ system acquisition. Each channel is represented by a fixed offset. . . . . . . . . . . . . . .

B.1 A reproduction of the programmatic binning procedure. The shot bin is each row of the midpoint location matrix. The CMP bin width is minimized by taking the diagonal of each 3x3 Tx block. The full CMP fold of 9 traces occurs during cycle 3 . The mean of the bin represents the spatial coordinate and the range of the bin equals its width. . . 


\section{LIST OF FIGURES}

1.1 The 2016 (black) and 2017 (red) GreenTrACS route. The traverse departed from Raven/DYE-2 on May 3, 2016, and arrived at Summit Station June 6, 2016. Firn cores were drilled at field camps (numbered in panel b). During the 2016 campaign southern firn cores 1 - 7 were exhumed. In 2017 the traverse departed Summit Station on May 6, 2017, completed the clockwise circuit in West Central Greenland, and returned to Summit Station June 19, 2017. During the 2017 season firn cores 8 - 16 were exhumed. Radar surveys were conducted along the main route and spurs. Snow pits were excavated at drill sites and the eastern or western most ends of the radar spurs. The Core Site 15 location and nomenclature are diagrammed in panel c. The yellow highlighted spur route is examined within this thesis. Radar transects co-located with Operation IceBridge are indicated by the OIB tag. The map key indicates traverse direction, the routes traveled each year of the traverse, core site locations, and spur orientations at the 2016-2017 interchange location. . . . . . . . . . . . . . .

2.1 Constructing the 2017 radar system within the Science and Operations Building at Summit Station, Greenland. . . . . . . . . . . . 
2.2 Multi-offset radar under operation and repair during the 2016 GreenTrACS traverse. . . . . . . . . . . . . . . . . . . .

2.3 The 2017 multi-channel array featured a linked sled system which enhanced signal quality and increased mobility. . . . . . . . . .

2.4 This chart diagrams the work flow of the methodology. The red boxes highlight the individual components of the preprocessing. The yellow boxes contain more complex systems of the methodology. . . . . . . .

2.5 This chart diagrams the work flow of the methodology. The red boxes highlight the individual components of signal processing. The yellow boxes contain more complex systems of the methodology. . . . . . .

2.6 The cumulative distribution function and probability density function for the experimental determination of $\tau$. The simulations were conducted at 5 noise levels $\sigma=[\mathbf{1}: \mathbf{5}] \mathbf{d t}$. The PDFs indicate that for any noise level the solution converged within 25 iterations. The dashed black curves on the CDFs represent the ensemble mean. The black star is the determined value for $\tau ; 0.02$ for the LMO velocity model and 0.0055 for the interval velocity model. . . . . . . . . . 38

2.7 The two-phase CRIM model applied to dry snow/firn. Constants used and the CRIM equation are within the figure. . . . . . . . . 
3.1 The surface elevation above mean sea level for the Core 15 Spur W traverse. Post-processed GPS elevations relative to the WGS84 ellipsoid model have been corrected to the EGM2008 geoid surface to deliver elevations above mean sea level. Notice the trough nearest to the end of the transect. This topographic feature captures greater snow accumulation. . . . . . . . . . . . . . . . .

3.2 Offset gathers $\mathbf{4} \mathbf{~ m}, \mathbf{8} \mathbf{~ m}$, and $\mathbf{1 2} \mathbf{~ m}$ for the traversed route from Core 15 to the spur's end are annotated to identify the important wavefield features. The peak amplitudes of the picked horizons are blotted out in black and are identified with arrows. It is important when matching the initial reflection horizon phase across offset gathers to account for the reduction in cycles of the lateral waves bounded by the surface and reflection waves. . . . . . . . . . . . . . . . .

3.3 The 2016-2017 annual accumulation, average density, and depth of the annual reflection horizon are presented from the Core 15 Western Spur transect. Two density estimates are provided. The shallower density result is from the surface wave LMO velocity analysis and the deeper density stripe results from the reflected wave NMO velocity analysis. Uncertainties are expressed at the $1 \sigma$ confidence level. . . . . . . . . 
3.4 Plotted in black is the density log of GreenTrACS Core Site 15. The Herron-Langway model, plotted in red, estimates at depths matching those of core segments. In the lower left, the upper $3 \mathrm{~m}$ is enlarged to show the surface density clearly. Yellow ellipses display the multichannel radar estimates that drive the density model. Numbered: (1) the surface density, (2) the LMO density, (3) the forcing density for the Herron \& Langway (1980) model segments, (4) the NMO density. Uncertainties are expressed at the $1 \sigma$ level. The RMSE $=41.3 \mathrm{~kg} / \mathrm{m}^{3}$ between the MxHL model and core log. Much of the misfit can be explained by the Core 15 variance. . . . . . . . . . . . .

3.5 A cumulative average has been calculated for the Core Site 15 density $\log$ and the MxHL model. In the lower left, the upper $3 \mathrm{~m}$ is enlarged to show the integral averaged surface density clearly. The MxHL snow and firn compaction model is driven in the integrally averaged domain. A good fit exists RMSE $=7.3 \mathrm{~kg} / \mathrm{m}^{3}$. Variation of the Core 15 density $\log$ is removed by the vertical averaging. Uncertainty is measured at the $1 \sigma$ confidence level. . . . . . . . . . . . . . . . . 58 
3.6 The multi-channel radar derived result is first evaluated at Pit $15 \mathrm{~W}$, corrected for bias, and then evaluated at the Core 15 site. This figure presents the bias corrected result with the radar information (yellow ellipses) used to inform the MxHL snow and firn compaction model. The integral averaged density is sensed by the radar and Dix's conversion is used to retrieve the interval density. Due to wavelength limited resolution of the radar, the estimated density profile is a simple linear approximation of the snow density. . . . . . . . . . .

3.7 The stacked and depth converted radar section provides a smooth view of the snow and firn stratigraphy. Flat-lying reflecting horizons are isochronous. Reflections of the electromagnetic wave are caused by changes in density and pore structure of the shallow snow and firn. The continuity of reflections is degraded where the dip steepens near the transect end. This trough correlates with the surface topography

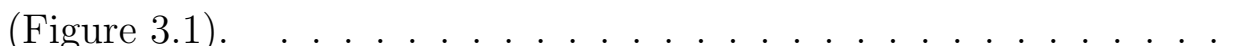

3.8 The mean from the ensemble of possible models within the $1 \sigma$ CI has been chosen to represent the density at depth. Peak amplitudes are overlain the density model with a flat gain. Spatially continuous IRHs are clearly observed at all depths. Amplitudes on the top of the section are muted to detail the subtle variation in surface density. . . . . . 
3.9 The mean density of each depth level is subtracted to evince anomalous spatial characteristics of the snow and firn density. Panel a: The density deviation of the zeroth depth level. Panel b: The two intersection points in the MxHL model are obviated at depths of $\sim 1.5 \mathrm{~m}$ and $\sim 12 \mathbf{~ m}$. The first leg of the model is driven by the multi-channel radar, and the second and third legs are predicted by the Herron \& Langway (1980) empircal model. The second breakpoint in the model occurs at the critical density $550 \mathrm{~kg} / \mathrm{m}^{3}$. The change in densification process at critical density correlates with a stratigraphic horizon. . . .

3.10 The Depth-Age relationship is predicted by the MxHL model and displayed as a function of depth and distance. Isochronous horizons are estimated at the summer surface for the given years (2016, 2014, 2010, $2005,2000)$. The 2000 summer surface is the deepest uninterrupted isochrone within the radar sounding. The 2016-2017 accumulation rate explored by the MxRadar drives the model. Accumulation rate is constant in the Herron \& Langway (1980) formulation. . . . . . . . . . 66

3.11 The modeled depth-age relationship is overlies the radiostratigraphydepth image. Qualitatively the modeled isochrones are a good fit the radar amplitudes. Greater misfit occurs at depth because lateral firn advection or migration of the surface trough upsets the steady-state assumptions of the Herron \& Langway (1980) prediction. The accuracy of this age model can be assessed via geochemistry analysis of Core 15. 67 
B.1 The ray paths for a shot and CMP gather through a homogeneous velocity medium. This figure emphasizes the different binning strategies for each respective gather. Notice the density and location of reflection points. . . . . . . . . . . . . . . .

B.2 Three groups of static traces were recorded in this data file. Subfigure a depicts the normalized covariance CDF, three peaks where the normalized slope correspond to the three pauses during recording, and the slope threshold identifying the first peak. Subfigure b displays the covariance associated with each trace sorted in recorded order. The shaded region is the rejection interval. Subfigures c and d display the near-offset radargram before and after static trace removal. . . . . . . 101

C.1 The median residual is compared for two frequency pass bands: the nominal frequency, $500 \mathrm{MHZ}$, and the frequency suggested by Gerlitz et al. (1993). Significant dipping structure is removed and the WOW is overcompensated for when filtered at $200 \mathrm{MHz}$. . . . . . . . . . 109

C.2 The trough separating the 'WOW' and the reflection signal was identified by Gerlitz et al. (1993). I have highlighted the WOW component in red. The targeted frequency stop is $\sim 200 \mathrm{MHz}$ or $\frac{2}{5} f_{o}$ and typically represents the cutoff frequency for 'WOW' attenuation. The yellow, filtered, signal approaches zero amplitude near the $\frac{2}{5} f_{o}$ threshold. . . 110

C.3 The noise removed during the stacking process. The noise matrix has AGC applied within a 50 sample window to amplify the noise for visual representation. This image does not depict the true noise level. . . . . 115 
C.4 Coherent instrument noise is removed by subtracting the trace median of overlapping spatial gates. Removal of the linear banding enhances the reflection quality. Dimming in the noise matrix around 50 ns reveals reflection information which is preserved through this process. In this example the median was subtracted from all travel times of the nearest offset $(1.33 \mathrm{~m})$ channel. . . . . . . . . . . . . . . . . . . . 116

C.5 (Panel a.) The offset gather at $9.33 \mathrm{~m}$ after De-WOW filtering, trace stacking, and $t^{2}$ scaling. Although this image has been processed to an acceptable quality uncorrelated noise persists along the spatial dimension. (Panel b.) The same gather as in (Panel a.) with the additional process of $\mathrm{f}-\mathrm{x}$ predictive deconvolution applied. Notice the smoothed reflecting horizons after random noise attenuation without loss of the firn structure. Travel times have been clipped for display. . . . . . .

D.1 The GreenTrACS 2016 Core Site 7 depth-density profile is represented as average velocity and rms velocity (Panel a.) by equations (D.22) and (D.11). Interval velocity (Panel b.) is estimated by supplying both $\mathbf{V}_{\text {avg }}$ and $\mathbf{V}_{\mathbf{r m s}}$ into Dix's equation (D.15). The interval densities extracted from Core 7 are transformed into EM velocities via the CRIM equation (Chapter 2.5). The estimation of $\mathbf{V}_{\mathbf{r m s}}$ agrees well with $\mathbf{V}_{\mathbf{a v g}}$, and each of these measures agree well with the core data observable by the low root-mean-square error (RMSE) statistic. The velocity axis has been exaggerated to make the small error visible. . . . . . . . . . 132 
D.2 Al-Chalabi (1974) provides the velocity heterogeneity factor (g) and stacking velocity bias (B) as a tool for understanding the relationship between $\mathbf{V}_{\text {avg }}, \mathbf{V}_{\text {rms }}$, and $\mathbf{V}_{\text {stack }} \ldots \ldots \ldots \ldots \ldots$

D.3 The rms velocity solution for the synthetic travel-time model of Core Site 7 is produced in Panel a. Dix's solution for the interval velocity model is represented in Panel b. The number of intervals has been decimated by a factor of two to stabilize the solution. The RMSE function and $\tau$ factor are presented in Panel c. for each iteration. Random noise was added from the normal distribution of $\mu=\mathbf{0}$ and $\sigma=\mathrm{dt}$

D.4 Random noise was added from the distribution of $\sigma=\mathbf{3 d t}$ for this randomly drawn simulation. The value $\tau$ is selected after iteration 5. However, a very similar convergence factor is produced after the first iteration. In practice this solution would have converged after a single iteration, though the RMSE gradient is still decreasing. This represents a drawback to the global choice for $\tau$. . . . . . . . . .

D.5 The high noise level selected randomly from a Gaussian distribution of $\sigma=\mathbf{5} \mathbf{d t}$ produces large errors in the recovered rms and interval velocity models. Convergence generally occurs at a higher value $\tau$ in higher noise regimes. Here, the RMSE of the interval velocity profile is not greatly improved by the $\mathbf{L}_{1}$ solution. . . . . . . . . . . . . . 138

D.6 The raypath of a critically refracted wave traveling through a homogeneous snow pack model. The wave is reflected at a layer boundary of the snow and is refracted upon exiting the snow surface. . . . . . . 


\section{LIST OF ABBREVIATIONS}

AGC automatic gain control

CDF cumulative distribution function

CI confidence interval

CIP common image point

CMP common midpoint

CPU central processing unit

CRIM Complex Refractive Index Method

DVL digital video logger

EGIG Expéditions Glaciologiques Internationales au Groenland

EM electromagnetic

f-x frequency-space

FFT Fast Fourier Transform

GNSS global navigation satellite system

GPR ground-penetrating radar GPS global positioning system
GreenTrACS Greenland Traverse for Accumulation and Climate Studies

GrIS Greenland Ice Sheet

HF high frequency

ICESat Ice, Cloud, and Land Elevation Satellite

IRH isochronous reflection horizon

IRLS iteratively reweighted least squares

LMO linear moveout

MWE meters of water equivalent

MxHL multi-channel radar HerronLangway density model

MxRadar multi-channel radar

NCAR National Center for Atmospheric Research

NEEM North Greenland Eemian Ice Drilling 
NMO normal moveout

OIB Operation IceBridge

PDF probability density function

RCM Regional Climate Model

RES radio echo sounding

RMO residual moveout

rms root-mean-square

RMSE root-mean-square error

Rx reciever

SLR sea level rise
SMB surface mass balance

Tx transmitter

UHF ultra-high frequency

VLMO linear moveout velocity

VNMO normal moveout velocity

Vrms root-mean-square velocity

Vstack stacking velocity

VHF very-high frequency

WARR wide angle refraction reflection 
CHAPTER 1:

\section{INTRODUCTION}

The Greenland Traverse for Accumulation and Climate Studies (GreenTrACS) is a multi-disciplinary study of recent changes in the surface mass balance (SMB) within the West Central percolation zone of the Greenland Ice Sheet (GrIS). Surface mass balance is the summation of mass gains (snow and rain accumulation) and mass losses (sublimation/evaporation and meltwater runoff) from the surface of an ice sheet. GreenTrACS spans the following scientific disciplines: snow and ice physics, geophysics and subsurface imaging, geochemistry, and atmospheric modeling.

In situ firn core and snow pit observations of water equivalent and depth-age relationships are essential for evaluating ice sheet surface coupled atmospheric models. The GreenTrACS traverse provided the opportunity to measure annual snow accumulation and firn compaction in the Western Greenland percolation zone (Figure 1.1) by extracting 16 shallow firn cores and excavating 42 snow pits (16 of which were sampled for chemistry). Because the snowmachine traverse was nomadic, we occupied 7 and 9 sites per year and additionally operated a suite of radar instrumentation to explore the snow and firn stratrigraphy between drill sites. Of the radar instruments deployed, a multi-channel $500 \mathrm{MHz}$ pulsed radar configured with multiple antenna separations has the ability to estimate the snow and firn density along track. 


\subsubsection{Directory of the Thesis Chapters}

Within the following sections of Chapter 1, I establish the motivation of the GreenTrACS campaign in Western Greenland, I provide a review of radar sounding in glaciers and ice sheets from historic studies to current practices of air, space, and ground based radar platforms for the applications of ascertaining accumulation, surface density, and firn compaction, and I emphasize the practice of wave speed estimation from ground-penetrating radar applications in the cryosphere with selected studies from the current literature. I develop the methodology for the multi-channel radar (MxRadar) as it pertains to deriving continuous snow and firn density and accumulation within this thesis (Chapter 2). I have evaluated the accuracy of the MxRadar approach at Core 15 and its western spur snow pit and discover spatial variability within the snow and firn compaction (Chapter 3). I discuss known caveats of the chosen application of geophysics for this analysis and assess the usefulness and limitations of the multi-channel radar utility within Chapter 4 . I conclude with a summary of the results and applications of the MxRadar product for future studies (Chapter 5). 
Greenland Traverse for Accumulation and Climate Studies 2016 - 2017

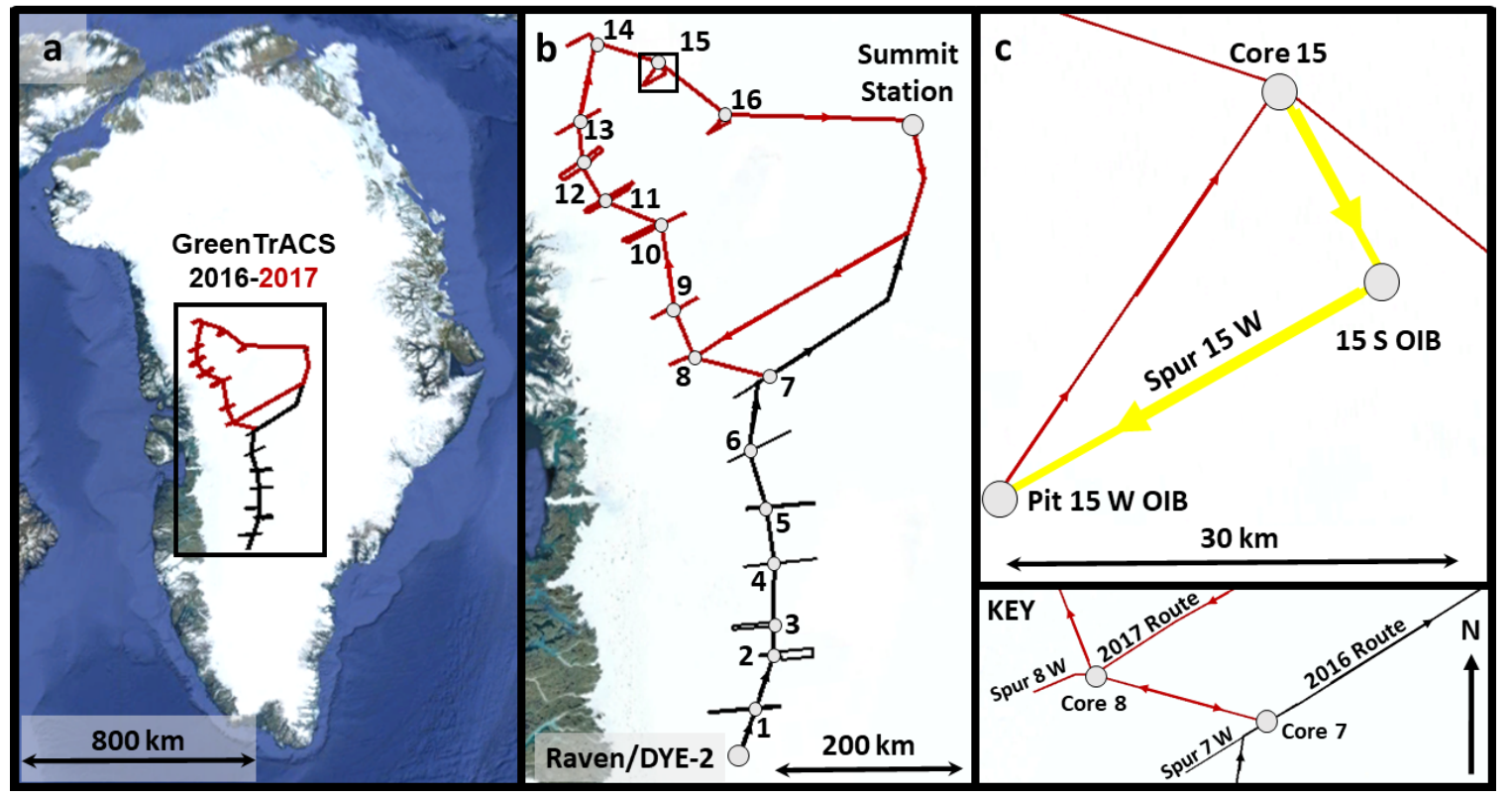

Figure 1.1: The 2016 (black) and 2017 (red) GreenTrACS route. The traverse departed from Raven/DYE-2 on May 3, 2016, and arrived at Summit Station June 6, 2016. Firn cores were drilled at field camps (numbered in panel b). During the 2016 campaign southern firn cores 1 - 7 were exhumed. In 2017 the traverse departed Summit Station on May 6, 2017, completed the clockwise circuit in West Central Greenland, and returned to Summit Station June 19, 2017. During the 2017 season firn cores 8 - 16 were exhumed. Radar surveys were conducted along the main route and spurs. Snow pits were excavated at drill sites and the eastern or western most ends of the radar spurs. The Core Site 15 location and nomenclature are diagrammed in panel $\mathrm{c}$. The yellow highlighted spur route is examined within this thesis. Radar transects co-located with Operation IceBridge are indicated by the OIB tag. The map key indicates traverse direction, the routes traveled each year of the traverse, core site locations, and spur orientations at the 2016-2017 interchange location. 


\subsection{Motivation for Advancing the Study of}

\section{Greenland Mass Balance}

Climate is a perceived indicator of quality of life and has a significant contribution, along with socioeconomic factors, for human settlement (Gamble et al., 2008). Prompted by global warming during the $21^{\text {st }}$ century for the likely range of temperatures (between 1.5 and $4{ }^{\circ} \mathrm{C}$ ), Alfieri et al. (2017) forecast increased flood risk worldwide. Alfieri et al. (2017) anticipate $\sim 250$ million people per year will be affected by flooding in year 2100, which follows an exponential trend from the present baseline of 54 million people affected per year.

The Greenland contribution to global sea level rise (SLR) has increased from 0.13 to $0.74 \mathrm{~mm} \mathrm{a}^{-1}$ during 1993 to 2005 (Bindoff et al., 2007). Since 2005, GrIS SLR contribution continued to accelerate with peak GrIS annual sea level contribution of $1.2 \mathrm{~mm}$ occurring in 2012 after unprecedented surface melting (Van Den Broeke et al., 2016; Nghiem et al., 2012). In 2013 the SMB contribution of the GrIS mass budget rebounded to levels that have not occurred since 2000; however, the SMB trend for 2013 to 2015 decreased at a rate greater than that of 2000 to 2012 (Van Den Broeke et al., 2016). Western Greenland displayed the largest regional acceleration in mass loss from 2002-2015, which is primarily due to changes in accumulation and melt and secondarily due to increased ice discharge (Van den Broeke et al., 2009; Sasgen et al., 2012; Van Den Broeke et al., 2016).

SMB is commonly estimated at regional scales using high-resolution regional climate models (RCMs) (Box, 2013), which rely on atmospheric reanalysis models (Chen et al., 2009). For the GrIS, climate data available for reanalysis is sparse and 
relies heavily on outdated accumulation measurements of the 1997-1998 Program for Arctic Regional Climate Assessment campaign (Abdalati \& Thomas, 2003). GreenTrACS provides much needed updates to the reanalysis consortium and will independently forecast regional climate to improve prediction the GrIS mass balance.

Surface mass balance may also be estimated from air or space-borne surface altimetry (McConnell et al., 2000). Correcting for changes in surface elevation by snow compaction without mass loss contributes positively to the altimetry retrieved SMB estimate (Zwally \& Li, 2002; Li \& Zwally, 2011; Sørensen et al., 2011). Measuring, understanding, and modeling snow and firn compaction continues to improve with the latest generation of Community Earth Systems Models (van Kampenhout et al., 2017). Yet, surface density forcing of ice sheet surface coupled atmospheric models remains simplistic and has not been evaluated by a continuous spatial method. The multi-channel radar traverse of the GreenTrACS campaign independently evaluates snow and firn compaction to deliver estimates of annual snow accumulation. A vast amount of radar sounding has been conducted on polar ice sheets, typically for the objective to ascertain accumulation rates and fewer to examine snow surface density and compaction. In the following section (1.2), I review the history of radio echo sounding and the application of radar methods to quantify snow and ice properties.

\subsection{Radio Echo Sounding of Ice Sheets}

Radar methods first determined the thickness of temperate arctic glaciers. The early radar was experimental but proved viable compared to contemporary seismic methods on Seward Glacier during the 1948 and 1949 traverse campaigns supported by the Office of Naval Research (Steenson, 1951). The equipment was a modified pulse type radio altimeter and was operated with antennas buried in the firn and suspended 
above the surface. An arduous two stations were recorded per day. Steenson (1951) derives the real and imaginary dielectric loss components for the reflection coefficient at normal incidence for the steady state, plane wave solution to Maxwell's equations. He provides mixing formulae for the effective dielectric of a glacier, given the volume fraction of ice, air, and water. Meltwater was present (estimated at 1.2\%) within the shallow firn of Seward Glacier during the summer campaigns. Steenson (1951) discusses difficulties when determining ice thickness from radio ranges in the presence of meltwater. Ground based radar methods developed and discussed in this early work are relevant in the studies of today.

The airborne application of radar for imaging the stratigraphy and thickness of continental ice was developed by the U.S. Army Signal Research and Development Laboratory prior to the International Geophysical Year 1957-1958. The transparency of ice to radar altimeters misled aircraft to accident and opened investigations to leverage the radio properties of ice for glacier sounding (Waite \& Schmidt, 1962). These early ground based and airborne surveys gave birth to the field of radio echo sounding (RES). Plewes \& Hubbard (2001) review the evolution of RES equipment, practices, and applications to glaciological studies.

\subsubsection{RES Studies of Accumulation, Surface Density, and Firn Compaction}

After much technological advancement, a suite of wideband radar instruments are flown during NASA's Operation IceBridge (OIB) mission to monitor the Greenland and Antarctic ice sheets and sea ice. The OIB mission provides RES, altimetry, and photogrametry to extend the observational record of earth's polar regions between NASA's Ice, Cloud, and Land Elevation Satellite (ICESat) 2003 - 2010 mission and 
its advanced generation ICESat-2. The ultra-high frequency (UHF) band (600 MHz to $900 \mathrm{MHz}$ ) Accumulation Radar of Lewis (2010) images firn and ice stratigraphy at large spatial scales. Snow accumulation rates for the past 100-300 years have been estimated by GreenTrACS researchers from Accumulation Radar imagery and are used to evaluate three regional climate models (Lewis et al., 2017). Though, to ascertain depths or annual thickness, electromagnetic (EM) wave velocity must be calibrated from dielectric permittivity measurements of ice cores. Benefits of this approach are gained when selected ice cores have been dated by glaciochemical methods (Legrand \& Mayewski, 1997).

Radio echos from buried depositional surfaces within the dry-snow zone are isochronal (of equal age). The continuous nature of snow, firn, and ice radiostratigraphy allows for age extrapolation over large distances. Age-depth relationships thereby provide accumulation rate measures. Alternative to chemical analysis, OIB wideband microwave radar (Panzer et al., 2013) imagery was used to develop annual stratigraphy. The age-depth relationship derived from the radiostratigraphy was as accurate as that derived from glaciochemistry of a nearby Antarctic firn core on Pine Island Glacier (Medley et al., 2013). Provided a velocity model calibrated from the average of 9 nearby firn cores, snow accumulation rates were accurately measured and showed no trending deviation from the mean accumulation of the past $\sim 30$ years. Hawley et al. (2006) determined annual layers in the dry-snow zone of the GrIS using imagery of the European Space Agency ASIRIS synthetic aperture interferometric radar altimeter. The authors calibrated the annual stratigraphy to hard winter layers evident by a neutron density log. The high-resolution (1 cm intervals) density profile agrees with the firn compaction model developed by Zwally \& Li (2002). Firn densification 
creates an annual spike in winter layer density related to vapor migration established by a positive temperature gradient as cold October snow buries a warmed summer surface (Benson, 1960).

Simonsen et al. (2013) study the compaction of firn layers by examining the annual layers imaged during repeated passes of the ASIRIS radar system across the width of the GrIS along the Expéditions Glaciologiques Internationales au Groenland (EGIG) line in 2006 and 2008. Simonsen et al. (2013) develop a firn compaction model which incorporates densification by meltwater percolation, retention, and refreeze. Parameters of the firn model are driven by the Danish Meteorlogical Institute HIRHAM5 regional atmospheric climate model. The firn model is tuned by Monte-Carlo random walk inversion evaluated at the North Greenland Eemian Ice Drilling (NEEM) site within the northwest Greenland dry-snow accumulation zone and Flade Isblink ice cap, which endures significant annual ablation, located in northeastern Greenland. Good agreement is found between the density-depth and age-depth estimates. Simonsen et al. (2013) remark that HIRHAM5 overestimates annual accumulation at the coastal Flade Isblink site, while it underestimates accumulation at the inland NEEM site, and that the compaction model would improve by incorporating lateral movement of percolated meltwater before refreeze. The tuned firn model forced by RCM output along the EGIG radar altimetery transect revealed some disagreement between radiostratigraphic isochronology and the modeled annual firn layering. Few annual layers were observed in the percolation zone by radar measurement. Firn compaction estimated by the repeat measurements of the ASIRIS was inconclusive due to signal limitations of the instrument.

To better classify landing sites for probe and rover instruments and future human 
exploration of planetary bodies, Grima et al. (2012) developed a stochastic determination between coherent and incoherent power returns from the surface echo of a 20 MHz martian orbital shallow radar. As an analogue to the icy surface of Jupiter's moon Europa, Grima et al. (2014a) apply the radar power retrieval method to a High Capability Airborne Radar Sounder survey of Thwaites Glacier, Antarctica. Using an analytical backscatter model, Grima et al. (2012, 2014a) estimate the surface roughness and dielectric constant. The coherent and incoherent power of the surface echo depends on the Fresnel surface reflection coefficient and root mean square surface height roughness. The Fresnel reflection coefficient at nadir is a function of the real dielectric permittivity of the atmosphere $(\varepsilon=1)$ and the icy surface. The ratio of the coherent power to the incoherent power is independent of the Fresnel coefficient - and thereby the surface parameters can be estimated. The retrieved dielectric permittivity is transformed into a measure of firn density by the formulation of Looyenga (1965), where, the half-width of the compressed $60 \mathrm{MHz}$ chirped signal with $15 \mathrm{MHz}$ bandwidth is representative of the first $6-8 m$ of the firn surface. Grima et al. (2014b) calibrate this bulk density measure to a firn core extracted within the Thwaites survey network to estimate the zeroth depth level surface density. Across $\sim 500 \mathrm{~km}$ of Thwaites, the surface density profile strongly correlates with snow accumulation estimated by Medley et al. (2013).

\subsubsection{Ground-Penetrating Radar Applications in Polar Firn}

Ground-penetrating radar (GPR) is widely used for estimating snow accumulation on continental ice sheets. GPR operates in the very-high frequency (VHF) to ultra-high frequency (30 MHz to $3000 \mathrm{MHz}$ ) range. The signal bandwidth is inversely proportional to the duration time of the radar pulse. The voltage pulse function is 
typically a Gaussian pulse which requires the impulse response of the antenna to be compact (Daniels, 2009). GPR energy typically radiates from resistively-loaded dipole antennas, chosen for their size and weight characteristics (Poley, 2010). Resistivelyloaded antennas have improved wideband performance by attenuating current at the antenna end as to not distort the input function or generate time domain side lobes (Daniels, 2009; Poley, 2010). The short pulsed waveform established by this voltage function and antenna is a 1.5 cycle Ricker wavelet. GPR often employs a single transmitter and receiver antenna pair at a fixed offset to record echos from firn stratigraphy, but multi-channel configurations allow GPR to simultaneously record swath volumes, multiple frequencies, and/or multiple antenna offsets.

Traversed glaciology studies present the opportunity to acquire validation data concurrent with radar profiling. In situ observations, either firn cores or snow pits, allow for estimates of the EM velocity - and thereby time to depth conversion, velocity to density conversion, and accumulation calculation. Maurer (2006) provides one of the initial GPR contributions for Greenland with 3-D imaging of the spatial variability of annual horizons and accumulation at two $1 \mathrm{~km}^{2}$ study sites that are apart of the larger Greenland Climate Network. Brown et al. (2011) conducted two studies within the percolation zone of Western Greenland, along the EGIG line. Within two survey grids $\left(775 \mathrm{~m}^{2}\right.$ and $\left.400 \mathrm{~m}^{2}\right)$ many $\sim 10 \mathrm{~m}$ firn cores were extracted and analyzed for stratigraphy. The spatial continuity of melt-refreeze stratigraphy was examined by 3-D radar imagery. While imaging continuous stratigraphy has proven difficult within the percolation zone due to increased firn heterogeneity, Brown et al. (2011) show that multi-annual melt features from $\sim 1660$ masl are more continuous than lesser percolation features at Crawford Point ( 2000 masl $)$. 
A part of the International Trans-Antarctic Science Expedition, Arcone et al. (2004) traversed Western Antarctica with 400 MHz GPR along several transects hundreds of kilometers in distance. They explored the continuity of reflection horizons, factors which interrupt the radiostraigrahy, and physical processes which give rise to radio echos deep within the firn column where the relative change in density is small. Three isochronal reflections at depths greater than $30 \mathrm{~m}$ were traced throughout their survey. These reflections were dated by counting annual layers from a firn core that intersects the radar traverse. Arcone et al. (2004) hypothesize that radar returns from deep firn are caused by a series of thin 1-2 $\mathrm{mm}$ ice layers and polarity reversals of the signal are the result of buried hoar layers. The authors document long continuous radiostratigraphy profiles and report that folds imaged within the firn are likely caused by accumulation rate variability associated with wind direction and surface slope angle and aspect.

Ng \& King (2011) view stratigraphic undulations as waveforms and clarify, "undulations are not folds in the geological sense of having been created by force-bearing deformation in the stratigraphy, but kinematic waves resulting from differential mass transport." The authors have developed a kinematic wave theory which explains isochrone evolution observed in airborne radar and GPR imagery. Their model can retrieve snow accumulation rate and surface density from radar soundings parallel to the direction of ice flow under assumptions of time invariant flow and accumulation rate and spatially invariant firn density. To account for the depth-dependent submergence rate of isochrones as a function of density the authors use Sorge's law (Bader, 1954). Ng \& King (2011) note that the assumption of spatiotemporal steady state density leads to inaccuracy in the depth imaging of radiostratigrpahy. $\mathrm{Ng} \&$ 
King (2011) state that sparse EM velocity estimates (derived from firn cores or radar methods) do not contribute to improving this assumption. This entails inaccuracies within inversely solved accumulation records. Ng \& King (2011) suggest coupling the time dependent vectorial ice flow field to their firn compaction model to alleviate some of the steady state assumption, though continuous spatial measurement of firn density is excluded.

\subsubsection{Electromagnetic Velocity Determination of Glaciers: Applications of GPR}

The EM-velocity of snow and firn can be measured using GPR. Velocity determination by GPR methods aims to be independent from additional in situ measures by exploiting characteristics of the recorded wavefield. Using a GPR with a common offset between transmitting and receiving antennas at various look angles, Taurisano et al. (2006) map ice cracks, voids, and larger crevasses along a Norwegian supply route on Fimbul Ice Shelf, Antarctica. Sharp velocity discontinuities in the subsurface diffract propagating waves in all directions and return as hyperbolas on the GPR recording. The average velocity of the medium above a diffraction can be estimated from the hyperbolic travel-time moveout curvature. Migration collapses a diffraction hyperbola when the correct velocity is applied. Known as migration velocity analysis, EM velocity can be determined by testing many possibilities and choosing the velocity which mitigates the diffraction. Bradford \& Harper (2005) use wavefield migration velocity analysis as a tool to measure water content within Bench Glacier, Alaska. Because the EM velocity of water is $\sim 5$ times slower than pure ice, freshwater occupying the glacier fluid pathways (pore space, voids, and cracks) creates velocity variations which are sensed through the analysis of GPR. 
EM velocity can also be determined by the common midpoint (CMP) survey. CMP reflections can be acquired at stations by recording a trace, increasing the horizontal distance between antennas, and repeating the measurement about a central reflection point for many offset separations. Alternatively, multi-channel GPR systems that use an array of antennas with various fixed offsets between source(s) and receiver(s) allow for continuous CMP profiling. EM velocity can be retrieved by several methods of CMP analysis that I will review.

Eisen et al. (2002) describe the stationary CMP methodology in detail and test the validity of the CMP velocity determination at four radar frequencies: 25, 50, 100, and $200 \mathrm{MHz}$. The authors apply the short-spread normal moveout equation and determine velocity using a coherence method (Neidell \& Taner, 1971). In Chapter 4.1 I discuss several assumptions in this reflection travel-time equation, which have implications for the accuracy of the method. Eisen et al. (2002) find a trend of increasing error in the velocity estimate with decreasing frequency. The authors attribute errors to violation of the short-spread approximation and inaccuracy of the depth estimate due to increased wavelength at lower frequency.

At 13 sites within the western percolation zone of the GrIS along the EGIG line spanning the elevation range $\sim 1400-2000$ masl, Brown et al. (2012) conducted stationary CMP surveys. Travel-time curves were picked on the CMP gathers at each site deep enough to measure glacial ice (firn density exceeding $830 \mathrm{~kg} / \mathrm{m}^{3}$ ). The EM velocity profile which best fits the travel-time data is solved iteratively by damped least-squares inverse methods using a ray-based forward model and imposing linear constraints on the subsurface velocity. The travel-time inversion does not rely on the assumptions of normal moveout. At Crawford Point ( 2000 masl $)$ a 120 m firn 
core was drilled to confirm the accuracy of the CMP velocity analysis. Brown et al. (2012) compare their results at the lower elevation sites to the Herron \& Langway (1980) density model. They show that firn thins and increases in density within the percolation zone as elevation decreases. The densification of firn by meltwater percolation and refreeze is not represented in the compressive densification model of dry snow by Herron \& Langway (1980).

Brown et al. (2017) conducted radar surveys at high frequencies (HF) (2.5, 5, and $10 \mathrm{MHz}$ ) to sound the ice sheet thickness within the ablation zone of western Greenland $\sim 27 \mathrm{~km}$ inland from the ice sheet terminus. A common offset profile was conducted at each frequency between two repeated hot water drilling locations $(\sim 820-850 \mathrm{masl})$ with average depths to bedrock measured downhole at $461 \mathrm{~m}$ and $698 \mathrm{~m}$. Thermistors were deployed downhole and allowed to equilibrate with the ice before retrieval. The temperature profile revealed a thicker cold ice layer and a temperate ice layer with thickness of $\sim 150 \mathrm{~m}$. An internal reflection observed on the common offset profiles coincides with, but is not necessarily caused by, this temperature boundary. A common source-point multi-offset (shot-gather) GPR survey was conducted with offsets greater than $800 \mathrm{~m}$. The travel-time inversion strategy described in Brown et al. (2012) estimated a two-layer EM velocity model; the upper cold layer with a negligible volume content of water and the wet temperate basal layer. Bulk velocity was also estimated with a radar sounding at the borehole locations, constrained by depth to the bed-rock reflection. Volumetric water content was estimated for each layer using the Looyenga (1965) empirical dielectric mixing formula and the Complex Refractive Index Method (CRIM) (Chapter 2.5). The CRIM formulation was favored in their study as estimates of englacial water content were more conser- 
vative and corroborated with wetness values reported by other studies. Ice thickness interpreted from Operation IceBridge Multi-channel Coherent Radar Depth Sounder imagery, where flight lines intersect the GPR profile recorded by Brown et al. (2017), is underestimated because of the water content within the glacier ice.

Multi-channel GPR configured with multiple antenna offsets was applied to estimate the volumetric water content of temperate Bench Glacier, Alaska (Bradford et al., 2009a). The multi-channel acquisition strategy allows for continuous CMP profiling. Bradford et al. (2009a) use a combination of pre-stack depth migration and travel-time tomography inversion to solve the EM velocity (Jones et al., 2008). The velocity analysis begins with a starting model that is derived form normal moveout analysis or extrapolated from borehole measurement. Depth migration is applied to the common-offset gathers. If the migration velocity is correct a given common image point (CIP) will maintain the same depth across all channels. Residual moveout (RMO) exists if the CIP deviates from flat and indicates inaccuracy of the velocity model to this depth. Perturbations to the velocity model which flatten any RMO are manually chosen for each reflection horizon. Lastly, the velocity model is updated by reflection-travel time tomograhpy. This process is computationally and labor intensive and can be applied iteratively until an accurate velocity is estimated. Bradford et al. (2009a) transform the EM velocity model by the three phase CRIM petrophysical model (ice, air, water). Bradford et al. (2009a) develop a model for volumetric air content that accounts for the compressive minimization of air bubbles within glacial ice. The air fraction model is coupled to the CRIM formulation to better estimate volumetric water content. Their analysis of multi-channel GPR is a refinement of their earlier study of Bench Glacier (Bradford \& Harper, 2005). 


\subsection{Implications of this Study for the Wider Scientific Community}

The conventional quantification of snow and firn - drilling and retrieving cores and digging snow pits - are strategically located point measures intended to represent the average of local and regional climate. A continuous spatial quantification method is able to assess the representative accuracy of sparse in situ data. Currently, variability in SMB components within a modeled grid cell is not applied within the National Center for Atmospheric Research (NCAR) Community Land Model; fluctuations in accumulation between 0 - $1 \mathrm{~m}$ w.e. do not contribute to the SMB passed into the NCAR Community Ice Sheet Model (Oleson et al., 2013). This is evidence of the disparage between the scale of coupled ice sheet atmospheric models and the capabilities remote sensing which have higher spatial resolution than their large scale modeling counterparts. High resolution modeling ( $\sim 1 \mathrm{~km}$ grid cell) is demanded at the grounding line of oceanic ice sheet termini for global climate model predictions of future SLR. To better couple with global atmospheric simulations at $1^{\circ}$ grid resolution, continental ice is treated with computationally efficient elevation discretizations (Lipscomb et al., 2013). For reference a $5 \mathrm{~km}$ resolution occupies $\sim 100$ additional grid cells per $1^{\circ}$ in Greenland (Lipscomb et al., 2013).

The traversed multi-channel GPR co-operates with the conventional in situ snow and firn observations to continuously quantify density, depth, and accumulation between excavation sites. The multi-channel radar technique developed within this thesis provides horizontal resolution of $\sim 2.5 \mathrm{~m}$ along track. I estimate the snow surface density and accumulation to constrain the prediction of density and accumulation at 
depth. This approach is necessary as in situ observations of surface processes can better inform remotely sensed and modeled glaciological processes that aim to improve estimates of surface mass balance (Van Den Broeke et al., 2016). The necessity for the continuous resolution of firn processes is not yet matched by computational resource or the accuracy demands of climate scenarios beyond $2100 \mathrm{CE}$. Through the advancement in the accumulation product by the MxRadar technique, a grid cell resolution less than $5 \mathrm{~km}$ is required to capture the significant spatial fluctuations of firn density and snow accumulation in western Greenland. Alternatively, the SMB components provided by the MxRadar technique can be upscaled.

Of the studies applying GPR for velocity analysis within the current literature, none have contributed continuous estimates on the scale of tens of kilometers. GreenTrACS multi-channel radar covers thousands of kilometers. Continuous velocity analysis of high-resolution, multi-channel GPR is a hulking task which pushes the limits of commercially available GPR hardware. The best method of analysis differs with the study environment, desired project outcomes, computational resources, and above all data quality. Great effort was made to acquire quality GPR profiles through the harsh and diverse percolation zone of Western Greenland. The variability of weather and challenges of the traverse require unique interpretations of the geophysical data. The methods and analysis within my thesis are tailored to handle large data volumes and produce results that may be adopted within the climate reanalysis consortium and leveraged to improve the predictive index of historical and future climate scenarios. 


\section{CHAPTER 2:}

\section{METHODOLOGY: ACQUISITION, SIGNAL PROCESSING, VELOCITY ANALYSIS, AND SNOW/FIRN QUANTIFICATION}

The traversed multi-channel radar technique is able to independently quantify the snow and firn depth, density, and accumulation. This chapter provides the methodology and concepts of the data reduction from field acquisition to quantifying the snow and firn mass. This chapter contains five sections: acquisition of the multichannel radar (Section 2.1), preprocessing workflow (Section 2.2), signal processing workflow (Section 2.3), velocity analysis (Section 2.4), and quantification of snow and firn depth, density, and accumulation (Section 2.5). I present the fundamental equations and theory behind each process which comprise these sections.

\subsection{Traversed Acquisition of Multi-Offset Radar}

A multi-channel, multi-offset system exploits the travel-time delays that arise by increasing the horizontal separation of wavefield receivers from the energy source location on the surface. The shortest travel-time paths of the radio wavefront across all receivers in the array are picked to determine the wavefront moveout velocity. Two fundamental wavepaths are examined within this work: one-way wavepaths of 
laterally moving energy and two-way wavepaths of reflected energy. The measurable delay of the wavefront travel-time upon arriving at distant receiving antennas behave with linear moveout (LMO) and normal moveout (NMO), respectively (Section 2.4). When the assumptions of normal moveout are satisfied, the velocity measure is the root-mean-square velocity (Vrms) above any travel-time horizon and is referred to as the stacking velocity (Vstack) and normal moveout velocity (VNMO) within the context of exploration geophysics. I use the velocity measure to estimate of the snow/firn depth, density, and accumulation (Section 2.5).

The Sensors and Software pulseEKKO PRO ${ }^{\circledR}$ was deployed in a multi-channel, multi-offset configuration for the 2016-2017 GreenTrACS campaign. This radar unit is a commercially available system capable of recording across multiple channels. The digitizing and recording unit (DVL) of the pulseEKKO system is programmable with the important data acquisition parameters: two-way time window, temporal sample interval, spatial sample interval, and stack quantity. Table 2.1 contains the chosen acquisition parameters for the $500 \mathrm{MHz}$ radar system. Discussion about the importance of these parameters is provided in Appendix A.2.

The multi-channel digital equivalent time sampling adapter developed by Sensors and Software ${ }^{\circledR}$ allows for the sequential shooting of traces between antenna pairs. The chosen sequence is programmed into the DVL which activates the antenna pair upon each triggering. The recorded trace is concatenated to the raw data matrix composed of all previous traces as acquired by the sequencing. The GreenTrACS multi-channel array sequences through 9 channels (Table 2.2). One completed sequence is referred to, herein, as a cycle. 
Table 2.1: The acquisition parameters for the $500 \mathrm{MHz}$ multi-channel system. The two-way recording time window was varied between the two campaigns: 250ns during 2016 and 225ns during 2017.

\begin{tabular}{||c|c|c|c|c||}
\hline Frequency & TwT & $\Delta t$ & $\Delta x$ & Stacks \\
\hline $500 \mathrm{MHz}$ & $225-250 \mathrm{~ns}$ & $0.2 n s$ & $0.25 \mathrm{~m}$ & 4 \\
\hline
\end{tabular}

\subsubsection{Antenna Array Geometry Design}

The antennas of the pulseEKKO PRO are able to be distributed in an array of separations which are limited by the lengths of the signal carrying cables. Three transmitting and three receiving antennas form nine independent recording channels that are spread in a linear array. Each channel represents a horizontal transmitterreceiver (Tx-Rx) offset (Table 2.2). Antenna separations were chosen after finding the optimal recording parameters such that the minimum offset to depth ratio does not exceed 0.5 at the maximum retrievable sounding depth. Using a constant EM velocity model of $0.2 \mathrm{~m} / \mathrm{ns}$ and a two-way time window of $250 \mathrm{~ns}$ the maximum sounding depth at zero offset is 25 meters. A linear array geometry was designed to capture the moveout curvature through this range of sounding depths. The near-offsets are more sensitive to shallow reflections, and the far-offsets are more sensitive at depth where the observable travel-time moveout occurs more obviously with wider aperture. As a general rule of thumb for reflection sounding, the offset to depth ratio of 1 yields the best sensitivity and velocity accuracy for array processing. The uncertainty of the NMO velocity estimate increases at depths beyond the the farthest offset of the array.

The $500 \mathrm{MHz}$ resistively loaded dipole antennas are directional with a downward radiation pattern. The antennas are shielded from radio energy propagating from 
above and, to a significant degree, laterally. The high frequency pulseEKKO system was chosen for this investigation of the shallow firn because of their robust construction. The harsh conditions of 16-weeks traversing the Greenland Ice Sheet is strenuous on any equipment. With some repairs, thousands of kilometers of radio echos were recorded with little discontinuity.

The acquired data are raw and must be processed before quantitative analysis is conducted. The processing routine has two-stages: preprocessing and signal processing. Preprocessing extracts the metadata of the radar recording (the trace header) and sorts the recording into gathers for signal processing and velocity analysis. Preprocessing is introduced within the following section (2.2) and elaborated in Appendix B. Signal processing techniques are presented in Section 2.3 and given detail in Appendix C. After signal processing, further geophysical analysis of EM velocity, depth imaging, and snow and firn quantification occurs. 
Table 2.2: The cycle of the nine channel $500 \mathrm{MHz}$ system acquisition. Each channel is represented by a fixed offset.

\begin{tabular}{||c|c|c||}
\hline Channel & Tx-Rx & Offset $(m)$ \\
\hline 1 & $1-1$ & 4 \\
2 & $1-2$ & 8 \\
3 & $1-3$ & 12 \\
4 & $2-1$ & 2.67 \\
5 & $2-2$ & 6.67 \\
6 & $2-3$ & 10.67 \\
7 & $3-1$ & 1.33 \\
8 & $3-2$ & 5.33 \\
9 & $3-3$ & 9.33 \\
\hline
\end{tabular}

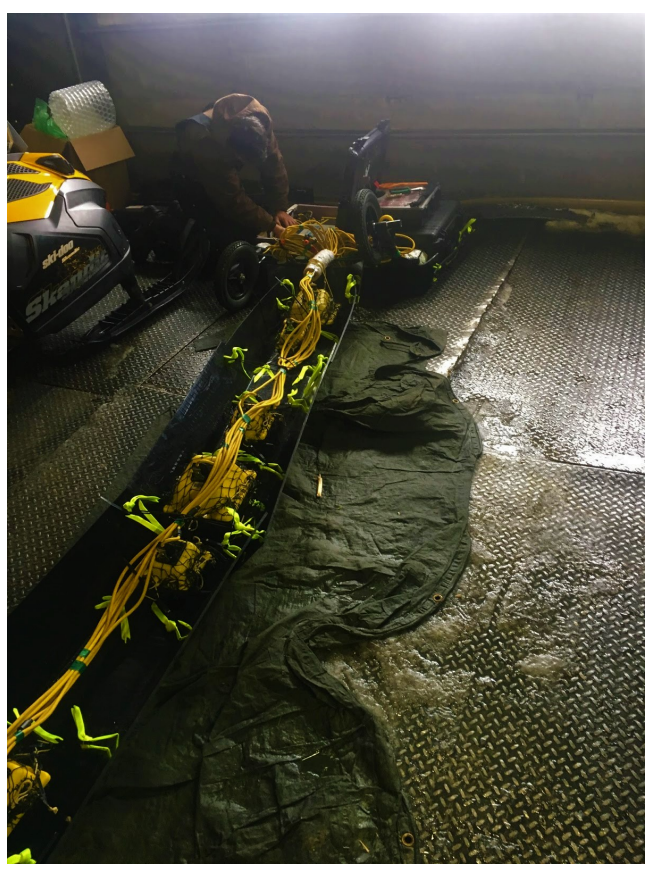

Figure 2.1: Constructing the 2017 radar system within the Science and Operations Building at Summit Station, Greenland. 

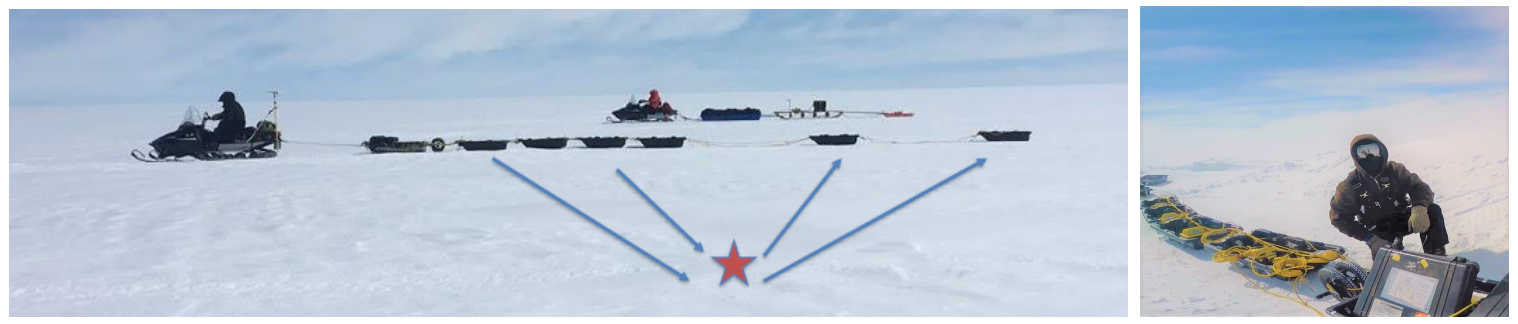

Figure 2.2: Multi-offset radar under operation and repair during the 2016 GreenTrACS traverse.

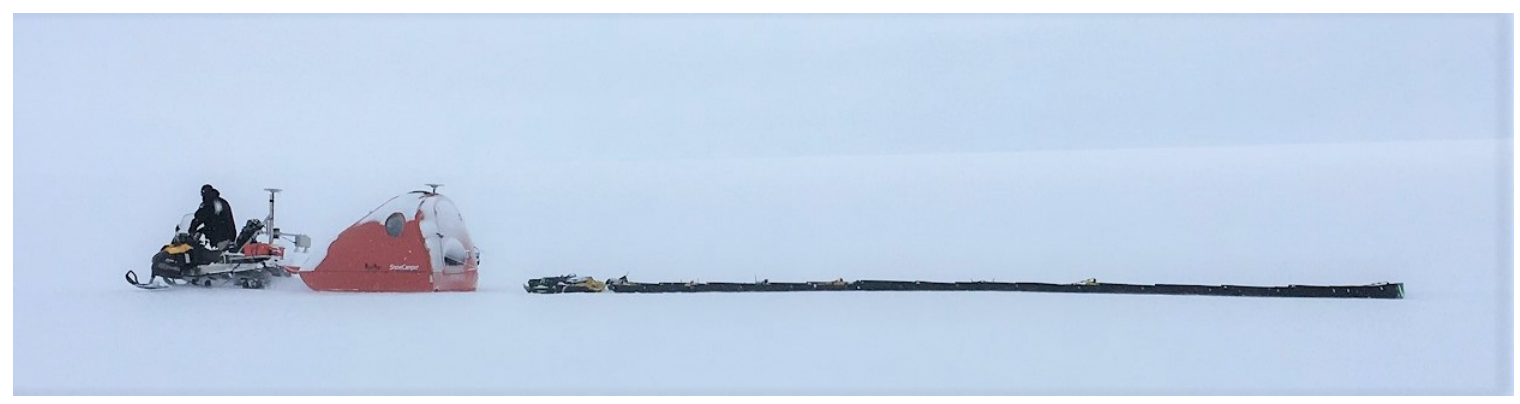

Figure 2.3: The 2017 multi-channel array featured a linked sled system which enhanced signal quality and increased mobility. 


\subsection{Preprocessing}

Before the acquired data are passed into the signal processing routine (Section 2.3), traces are sorted, edited, and georeferenced. The binary data and header files are passed into the MATLAB function ReadSensorsSoftwareData.m. This command parses the binary information into a matrix of raw amplitudes. The data matrix is in a multiplexed format. Columns of the matrix are the recorded traces that are appended sequentially by the antenna pairs. A structure of trace headers includes the necessary meta data of the recording: acquisition mode, trace index, channel number, nominal frequency, space and time sample interval, distance, and skip.

After the data has been loaded, the first job of preprocessing is to install the transmitter-receiver offsets - known as the array geometry. The array geometry (Table 2.2) is critical for the subsequent geophysical analysis. Next, the data is demultiplexed and sorted into individual matrices for each channel, or offset, known as common-offset gathers. Signal processing operates in the common-offset domain.

Duplicate traces occur in the data record as static traces (when operating in 'free run' acquisition mode) or as skipped traces (when operating in 'odometer' acquisition mode). Static traces accrue while the GPR is operating while stationary, and skipped traces occur when the array is towed too quickly. These unwanted traces are removed by an automated process. Traces are georeferenced after trace removal. The GPS was operated concurrently with the radar traverse. Because the sample interval of the GPS is less than the trace interval of the GPR, GPS information is interpolated so every trace has a geolocation. Similarly to static trace removal, stationary GPS locations are removed by an automated process prior to interpolation. 
The GPR waveform incurs sampling errors that incorrectly position the recorded trace in time. To accurately conduct further geophysical analysis I apply a systemic correction to shift the channels back to the correct position in time. This correction is a coarse scale calibration that aims to match the moveout velocity of the wavefront traveling laterally through the air to the free-space constant $(c)$. Additional residual corrections are applied during the velocity analysis (Section 2.4.3).

After these preprocessing steps the common-offset gathers are manipulated by digital signal processing routines. Then these data are resorted into common-midpoint gathers or common-shot gathers. The midpoint gather is a collection of all recorded offsets co-located about a single reflection point in the snow or firn. Traces sorted in the common midpoint domain obviate the travel-time functions of linear moveout and normal moveout. The velocity analysis routine operates in the common-midpoint domain. To recap, the prepossessing task is diagrammed in Figure 2.4. Elaboration on preprocessing is provided in Appendix B.

\section{Multi-Channel GPR Signal Analysis Flow: \\ Preprocessing}

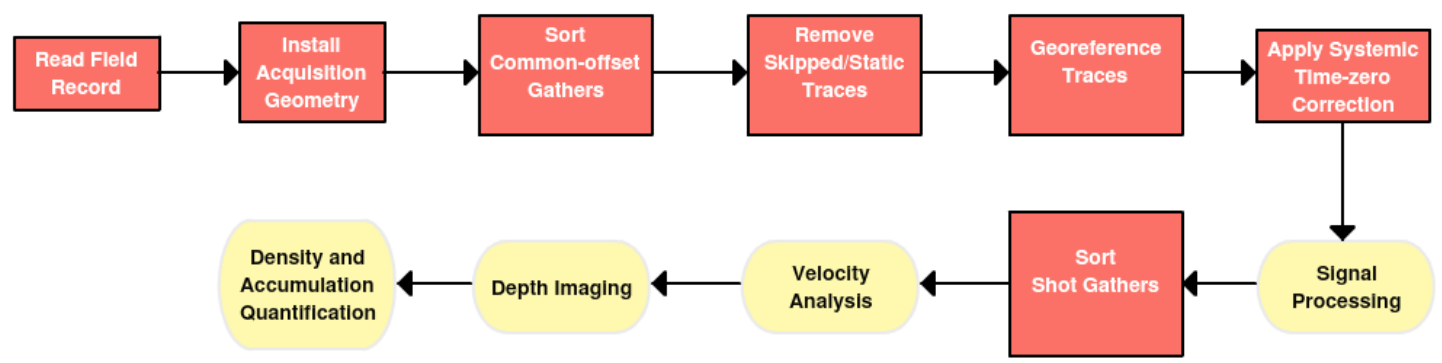

Figure 2.4: This chart diagrams the work flow of the methodology. The red boxes highlight the individual components of the preprocessing. The yellow boxes contain more complex systems of the methodology. 


\subsection{Signal Processing}

The primary goal of the signal processing routine is to eliminate random noise from the signal which arises from wavefield scattering and antenna bounce. The primary processing occurs in the common offset domain. The common-offset gathers are split among central processing units CPU and I have assigned one CPU per channel. The primary processing flow performs De-WOW filtering, amplitude gain, trace stacking, spatial median subtraction, and spatial predictive deconvolution to reduce the signal into a form which can be interpreted for horizon velocity analysis. The secondary processing goal incorporates the wavefield velocity information to perform normal moveout correction, stack midpoint gathers, and place the post-stack gather into the depth domain. Depth conversion enables relationships between the radiostratigraphy and nearby firn cores. The processed radar section is visually enhanced when colorscaled and presents smooth and continuous isochronous reflection horizons (IRH) for interpretations of mechanical firn compaction behavior and spatial mapping of snow accumulation. Appendix $\mathrm{C}$ elaborates on the implementation of the signal processing digital filters. The signal processing work flow is diagrammed within the methodological framework in Figure 2.5. 
Multi-Channel GPR Signal Analysis Flow:

Signal Processing

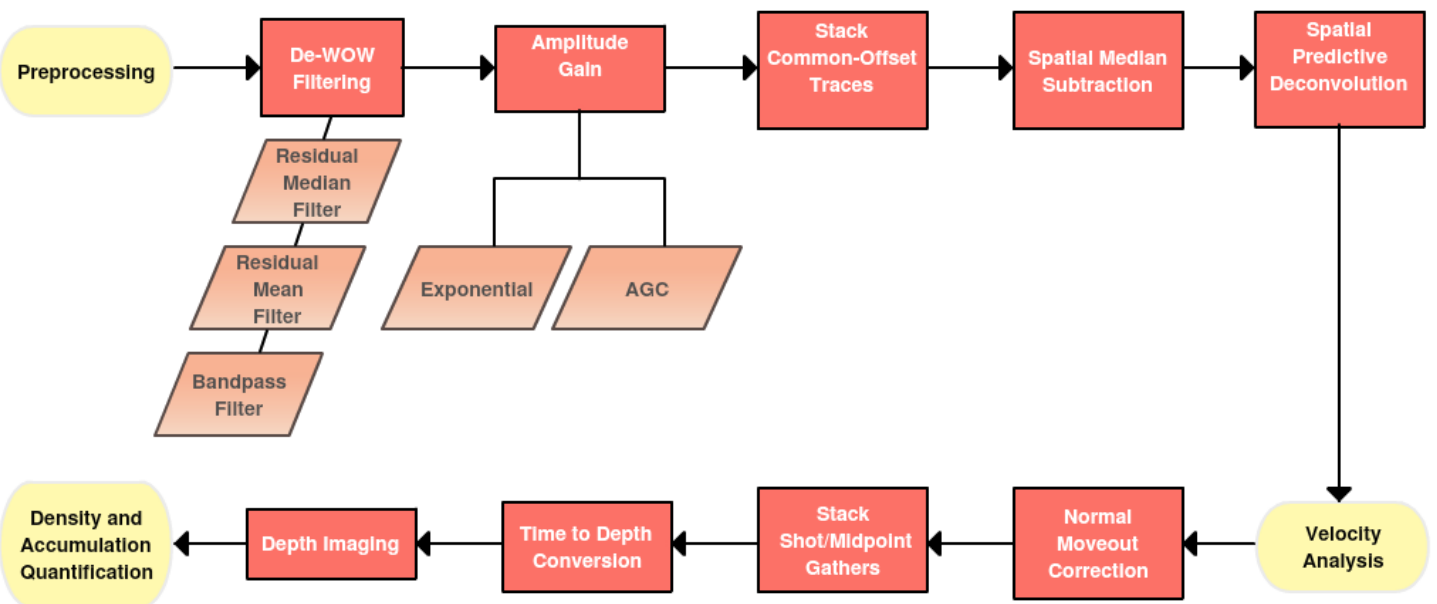

Figure 2.5: This chart diagrams the work flow of the methodology. The red boxes highlight the individual components of signal processing. The yellow boxes contain more complex systems of the methodology. 


\subsection{Horizon Velocity Analysis}

High quality, common-offset, radar images are output from the signal processing routine. These images contain the radar pulse travel-time information essential for estimating snow and firn accumulation. The application of multi-channel velocity analysis makes direct estimates of the overlying snow and firn density. Electromagnetic wave velocity, like depth or density, is a physical property of the snow and firn.

The analysis of the travel-times recorded by multiple antenna pairs is the crux of this methodology. I give much consideration to the proper selection of wavepath energy in the horizon velocity analysis (HVA) to exploit the moveout characteristics of each path. Three waves are apparent within the multi-channel record: the direct air-coupled wave, the direct surface-coupled wave, and primary reflected waves. I guide my velocity analysis of these wavepaths by selecting coherent returns with a semi-automated predictive picker (Appendix D.1).

To establish vernacular, I consider each spatially continuous lateral or reflected wavepath energy as a horizon, and I isolate these horizons as the following. The direct air-wave is chosen first. Identifying the air-wave is necessary to apply residual static corrections and establish the time-zero. The surface coupled wave is chosen second which provides velocity information about the zeroth depth level. The linear moveout velocity analysis of the direct air- and surface-coupled waves is detailed within Subsection 2.4.1. Internal reflection horizons appear in the data following the direct wave arrivals. These reflecting horizons are understood to be isochronic; each continuous reflector provides information of a specific depostional/erosional/metamorphic surface of a constant age. Aside, the isochronal relationship provides the means for 
deriving accumulation rate estimates (Section 2.5.4). The details of normal moveout velocity analysis for the internal reflection horizons are provided within Subsection 2.4.5.

\subsubsection{Linear Moveout Analysis of Direct Arrivals}

The direct wave arrives on a forward ray-path from the transmitting antenna to the receiving antenna. The travel-time delay of a wavefront as it travels from the transmitting antenna to the receiving antenna is known as moveout. The linear moveout behavior of direct waves is well understood and can be characterized by plotting the raw travel-time picks against offset (Burger et al., 2006, chap. 2). The direct wave moveout velocity (VLMO) and the zero-offset intercept time $\left(t_{0}\right)$ are the solution parameters of equation 2.1, where $x$ is the horizontal antenna separation $t$ is the recorded one-way, direct arrival time.

$$
t=t_{0}+\frac{x}{V_{L M O}}
$$

As the wavefront is emitted from the antenna, a ray bends through the media following the shortest travel-time path. This ray-path behavior is known as Fermat's Principle or the principle of least time (Burger et al., 2006, chap. 2). Rigorous computation of the optimal ray-paths are used in the seismic industry when determining the earth velocity model (Moser, 1991). I make the stead-fast assumption that the velocity of the medium surrounding the antennas is equivalent to that of free-space $c \sim 0.299 \mathrm{~m} / \mathrm{ns}$. I make further assumptions regarding the snow and firn velocity structure. I consider the snow and firn to be composed of homogeneous layers bounded by flat-lying interfaces. And I ignore out of plane scattering and reflecting. These assumptions hold well in my analysis, provided the slope consideration relative 
to the array length and the sounding depth made in Appendix A.2 and random noise attenuation procedures of Section 2.3.

\subsubsection{The Air-Coupled Wave}

The wavefront of the shielded Sensors \& Software ${ }^{\circledR}$ antenna is hemispherical with a downward radiation pattern. Unlike omni-directional antennas, the lateral moveout of the air-wave has low amplitude and is not easily apparent at farther antenna separations. To elucidate the air-wave arrivals I apply an AGC gain (Appendix C.2.3) prior to picking.

Abiding Fermat's Principle, the air-coupled wave has expected moveout at the free-space velocity with a small degree of variation. This fact is necessary to conduct the subsequent analysis because of systemic and random error in travel-time recordings during acquisition (Appendix B.7). Residual static corrections of this problem are discussed within Subsection 2.4.3. The intercept time estimated from the air-wave velocity analysis after residual static corrections is crucial for accurately estimating subsequent moveout velocities. The air-wave intercept time $\left(t_{0}\right)$ at zero-offset is a positive, non-zero, real number, which is descriptive of each shot gather. $t_{0}$ must be subtracted from the horizon picks of each shot gather. Because the travel-time information is a causal system, where reflectivity depends on past and present traveltimes, this is justified. Where $t$ is a chosen travel-time of a horizon (direct arrival or reflected arrival), $t^{*}$ is shifted earlier in time by the static shift (equation 2.2).

$$
t^{*}=t-t_{0}
$$

This procedure is often referred to as the time-zero correction. 


\subsubsection{Residual Systemic Static Correction}

A first pass estimation of the air velocity $V_{a i r_{c a l}}$ is conducted by least-squares regression (Subsection 2.4.7) using the global channel shifts found after field calibration. The field calibration air-wave arrivals display systemic and random errors discussed in Subsection B.7. The standard deviation of calibration travel-times are $\sim \pm 3$ samples and are approximately consistent among all channels. This residual is apparent after the first pass air velocity estimation. Solutions to the regression are typically $\sim \pm 0.01 \mathrm{~m} / \mathrm{ns}$ greater or less than the theoretical velocity. The travel-time residual vector $(\Delta t)$ for each channel is calculated in equation (2.3), where $O f f$ set is a vector of antenna separations calculated in equation (B.3) and $c$ is the universal velocity constant for electromagnetic wave propagation in free-space.

$$
\begin{aligned}
& \Delta t=\frac{O f f s e t}{V_{a i r_{c a l}}}-\frac{O f f s e t}{c} \\
& t^{* *}=t^{*}-\Delta t
\end{aligned}
$$

An individual $\Delta t$ correction is applied to each channel for every shot gather of the traverse (equation 2.4). After the timing corrections have been applied to the data and picked arrival times, the least-squares regression is recomputed to estimate the final velocity of the air-wave. When the appropriate $\Delta t$ correction is applied, the air-wave velocity is ensured to move out at speeds approximately equal to and not exceeding $0.299 \mathrm{~m} / \mathrm{ns}$.

\subsubsection{The Surface-Coupled Wave}

The EM velocity of the zeroth depth level can be estimated by examination of the surface-coupled direct wave. The zeroth depth, as I interpret this measurement, 
is the average wavelength of the surface wave. I calculate the depth level and velocity experimentally by picking various phases of the surface horizon and estimating the moveout velocity of the chosen wavelet phases by linear regression (Subsection 2.4.7). Where $V_{\text {surf }}$ is the surface wave LMO velocity and $t_{0_{\text {surf }}}$ is the static corrected intercept time, the experimental depth level

$$
z_{\text {surf }}=V_{\text {surf }} \cdot t_{0_{\text {surf }}}
$$

for many phases of the surface-wave are calculated by equation (2.5). The information from many chosen phases may be averaged, or only later or earlier phases may be chosen with the understanding that this measurement is averaged over the width of the pulsed wavelet. Though this experimental calculation is an approximation of the zeroth depth level, results tend to agree with observations recorded from snow pits (Chapter 3).

\subsubsection{Normal Moveout Analysis of Reflected Arrivals}

When sensed by the many receiving antennas of a midpoint gather, the reflection travel-time from a horizontal, flat-lying, and homogeneous snow or firn layer abides a hyperbolic moveout geometry known as normal moveout (Yilmaz, 2001, chap. 3). Because the shortest travel-time path within a homogeneous layer is a straight ray, the hyperbolic NMO equation is derived explicitly from the Pythagorean theorem. NMO velocity analysis exploits the travel-time information recorded by the array of antennas to measure the root-mean-squared ( $\mathrm{rms}$ ) velocity above a reflection horizon. The short-spread NMO equation (2.6) is the standard practice for determining an rms velocity model within a horizontally stratified earth (Yilmaz, 2001, chap. 3). $t$ is the 
recorded two-way travel-time by the receiving antenna at an offset distance $x$ from the transmitting antenna. Here, $t_{0}$ is the zero-offset intercept time of the NMO hyperbola. $V_{r m s}$ is the objective of this analysis.

$$
t=\sqrt{t_{0}^{2}+\frac{x^{2}}{V_{r m s}^{2}}}
$$

With respect to many of the known caveats of normal moveout analysis discussed within Chapter (4.1), I consider my solution of this problem adequate for measuring the firn density and accumulation. In the experiment of Appendix D.3.2, applying the shifted hyperbola NMO equation did not improve the result. This indicates that vertical velocity gradients within the firn are small and bolsters the use of equation (2.6) for HVA. The formulation of the higher-order NMO approximation is provided in Appendix D.2. The expansion term $S(x)$ (equation D.10) tended to destabilize the inverse solution of the velocity regression parameters. Hereby, I perform the velocity analysis using Dix's (1955) short-spread NMO approximation.

\subsubsection{The $x^{2}-t^{2}$ Method and Interval Velocity}

Green (1938) proposed a linear strategy to determine $V_{r m s}$ of a one layer earth model, known as the $x^{2}-t^{2}$ method. By squaring both sides of the hyperbolic equation (2.6), the NMO equation takes the form of a linear function (2.7).

$$
t^{2}=t_{0}^{2}+\frac{x^{2}}{V_{r m s}^{2}}
$$

When plotted on axes $x^{2}-t^{2}$, the travel-time data from a reflecting horizon can be estimated by a linear regression. Where $x$ are the measured antenna separations and $t$ are the measured two-way travel time for each antenna pair, the slope of the 
regression $\left(\frac{1}{V_{r m s}}\right)$ and the time axis intercept at zero-offset $\left(t_{0}\right)$ are the estimated solution parameters.

The one layer case is simplistic, though special, because the rms velocity is equivalent to the interval velocity of this layer. The interval velocity $\left(V_{\text {int }}\right)$ is the average velocity of a bounded, planar, and homogeneous layer. Dix (1955) provides a method for determining the interval velocity of $n$ layers composing a horizontally stratified earth. In the synthetic experiments (Appendices D.3.2 and D.4) and in the Core 15 W results (Chapter 3.3.2) I have applied Dix's (1955) formulation for interval velocity and subsequent firn interval density. The interval velocity when converted to density (Section 2.5) represents a section of the firn column, much like the segments extracted in a firn core. The bounded stratigraphic firn layers are not exactly homogeneous, and the interval velocity is the apparent velocity of the snow or firn layer that has been averaged throughout the layer thickness. A column of firn can be expressed by a smooth or piecewise linear velocity gradient. At greater depths the homogeneous approximation is better because the rate of firn densification slows as the density asymptotically approaches the density of ice. The resolution of velocity derived density profile depends on the number and time position of the picked horizons. I review Dix's (1955) method to solve the interval velocity in Appendix D.3.

\subsection{7 $\quad \mathrm{L}_{1}$ Minimization for Velocity Regression}

The moveout velocities of direct and reflected arrivals are the straight line slope of the respective linearized travel-time equations. Direct waves obey the linear traveltime equation (2.1) and reflected waves adhere to the linear form of the NMO equation (2.7). I write the travel-time functions as a set of linear equations which have the model form $\mathbf{G}$ and parameters $\mathbf{m}$. I refer to the direct wave model as $\mathbf{G}_{\mathbf{L M O}}$ and 
the reflected wave model as $\mathbf{G}_{\mathbf{N M O}}$ (equations 2.8 and 2.10). $m$ is the number of observations within each shot gather.

The parameter vector $\mathbf{m}$ of length $n$ contains the unknown quantities which the $\mathbf{L}_{1}$ regression aims to estimate: intercept time $\left(t_{0}\right)$ and slowness $(S)$; slowness is $\frac{1}{V}$. Defining slowness this way linearizes the problem so it can be written as a matrix equation. $\mathbf{m}_{\mathbf{L M O}}$ and $\mathbf{m}_{\mathbf{N M O}}$ are provided in equations (2.9) and (2.11).

$$
\begin{aligned}
& \mathbf{G}_{\mathbf{L M O}}=\left[\begin{array}{cc}
1 & x_{1} \\
\vdots & \vdots \\
1 & x_{m}
\end{array}\right] \\
& \mathbf{m}_{\mathbf{L M O}}=\left[\begin{array}{c}
t_{0} \\
S_{L M O}
\end{array}\right] \\
& \mathbf{G}_{\mathrm{NMO}}=\left[\begin{array}{cc}
1 & x_{1}^{2} \\
\vdots & \vdots \\
1 & x_{m}^{2}
\end{array}\right] \\
& \mathbf{m}_{\mathrm{NMO}}=\left[\begin{array}{c}
t_{0}^{2} \\
S_{r m s}^{2}
\end{array}\right]
\end{aligned}
$$

For the vector $\mathbf{d}$ containing the recorded travel-times for the respective moveout events, the linear system takes the form of equation (2.12).

$$
\mathrm{Gm}=\mathrm{d}
$$

I elect to estimate $\mathbf{m}$ using $\mathbf{L}_{\mathbf{1}}$ regression because of the robustness of the solution to outlying data. Discordant travel-time observations arise from systemic and random static errors and subsurface artifacts, which inherently violate the assumptions of NMO analysis. Though leveraging an inversion scheme to fit the short-spread NMO model is a work-around for broken NMO assumptions, I am confident by my discussion that the underlying mathematical model is acceptable (Chapter 4). The solution I 
implement minimizes the 1-norm of the data residual vector (equation 2.13).

$$
f(\mathbf{m})=\|\mathbf{d}-\mathbf{G} \mathbf{m}\|_{1}=\sum_{i=1}^{m}\left|d_{i}-\left(\mathbf{G m}_{i}\right)\right|
$$

"The 1-norm solution, $\mathbf{m}_{\mathbf{L}_{1}}$, will be more outlier resistant, or robust, than the least squares solution, $\mathbf{m}_{\mathbf{L}_{\mathbf{2}}}$, because (2.13) does not square each of the terms in the misfit measure" (Aster et al., 2013, p. 43). Equation (2.13) is a convex, but nondifferentiable function that has a sharp global minimum point. The nature of (2.13) makes it tricky to solve. Aster et al. (2013) provide the practical method of iteratively reweighted least squares (IRLS) which converges to an $\mathbf{L}_{\mathbf{1}}$ minimized solution through a series of weighted least squares problems (equations $2.14-2.21$ ).

The algorithm begins on the zeroth iteration $(k=0)$ by solving the least squares travel-time problem (2.12) to obtain a residual vector $\mathbf{r}$.

$$
\begin{aligned}
\mathbf{m}_{\mathbf{L}_{\mathbf{2}}} & =\left(\mathbf{G}^{\mathbf{T}} \mathbf{G}\right)^{-1} \mathbf{G}^{\mathbf{T}} \mathbf{d} \\
\mathbf{r} & =\mathbf{d}-\mathbf{G}_{\mathbf{m}_{\mathbf{L}_{2}}} \\
\mathbf{R} & =\operatorname{diag}\left(|\mathbf{r}|^{-1}\right)
\end{aligned}
$$

The objective is to minimize the absolute value of the model residual (2.17). Function minima occur at values where $f^{\prime}(m)=0$. To take the derivative of $(2.17)$, the gradient of the objective function is computed (2.18). This is set to zero and rewritten (2.19).

$$
\begin{aligned}
f(\mathbf{m}) & =\|\mathbf{r}\|_{1}=\sum_{i=1}^{m}\left|r_{i}\right| \\
\nabla f(\mathbf{m}) & =-\mathbf{G}^{\mathbf{T}} \mathbf{R} \mathbf{r}=-\mathbf{G}^{\mathbf{T}} \mathbf{R}(\mathbf{d}-\mathbf{G} \mathbf{m})=0
\end{aligned}
$$


$\mathbf{R}$ is a weighting matrix which contains $|\mathbf{r}|^{-1}$ along the diagonal and is zero elsewhere. $\mathbf{R}$ is updated with each new computation of the residuals per iteration. When a given residual $r_{i}$ has approached a tolerable limit close to 0 its weight is fixed. I choose the residual tolerance as the signal sample interval. The 1-norm minimized parameters are estimated by equation (2.20).

$$
\begin{aligned}
\mathbf{G}^{\mathrm{T}} \mathbf{R} \mathbf{G m} & =\mathbf{G}^{\mathrm{T}} \mathbf{R d} \\
\mathbf{m}_{\mathbf{L}_{1}} & =\left(\mathbf{G}^{\mathrm{T}} \mathbf{R G}\right)^{-1} \mathbf{G}^{\mathbf{T}} \mathbf{R d}
\end{aligned}
$$

The $\mathbf{L}_{\mathbf{1}}$ function is nondifferentiable at the minimum. This caveat of the 1-norm solution introduces the need to iteratively approach the solution minimum within some tolerance. The closure of an iteration of the IRLS scheme occurs after a test for solution convergence.

\subsubsection{Determining $\mathrm{L}_{1}$ Solution Convergence}

The velocity regression reaches an $\mathbf{L}_{\mathbf{1}}$ minimized solution when the convergence tolerance $\tau$ becomes larger than the ratio of the updated parameter space (2.21).

$$
\tau>\frac{\left\|\mathbf{m}^{\mathbf{k}}-\mathbf{m}^{\mathbf{k}-\mathbf{1}}\right\|_{2}}{1+\left\|\mathbf{m}^{\mathbf{k}}\right\|_{2}}
$$

$k$ is the iteration number which is updated after each computation of the parameters m during (2.20). $\tau$ is a dimensionless quantity that describes the ratio of the change in the length of the parameter space per iteration. Because $\tau$ is difficult to interpret, I ran simulations of the travel-time problem to experimentally determine the convergence factor $\tau$. The experiment is given detail within Appendix D.4. 
Figure 2.6 displays convergence values of $\tau$ and iteration index as the cumulative distribution function (CDF) and probability density function (PDF), respectively, for the snow pit and firn core models at each of five noise levels. The PDFs indicate that for any noise level the solution converged within 25 iterations. The dashed black line on the CDF plots represents the ensemble mean. I have selected $\tau$ at the $95 \%$ probability interval of the ensemble mean curve. $\tau$ equals 0.02 for LMO velocities and 0.0055 for NMO velocities. If the noise regime of observed travel-time picks is established, a case dependent $\tau$ may be selected from Figure 2.6.
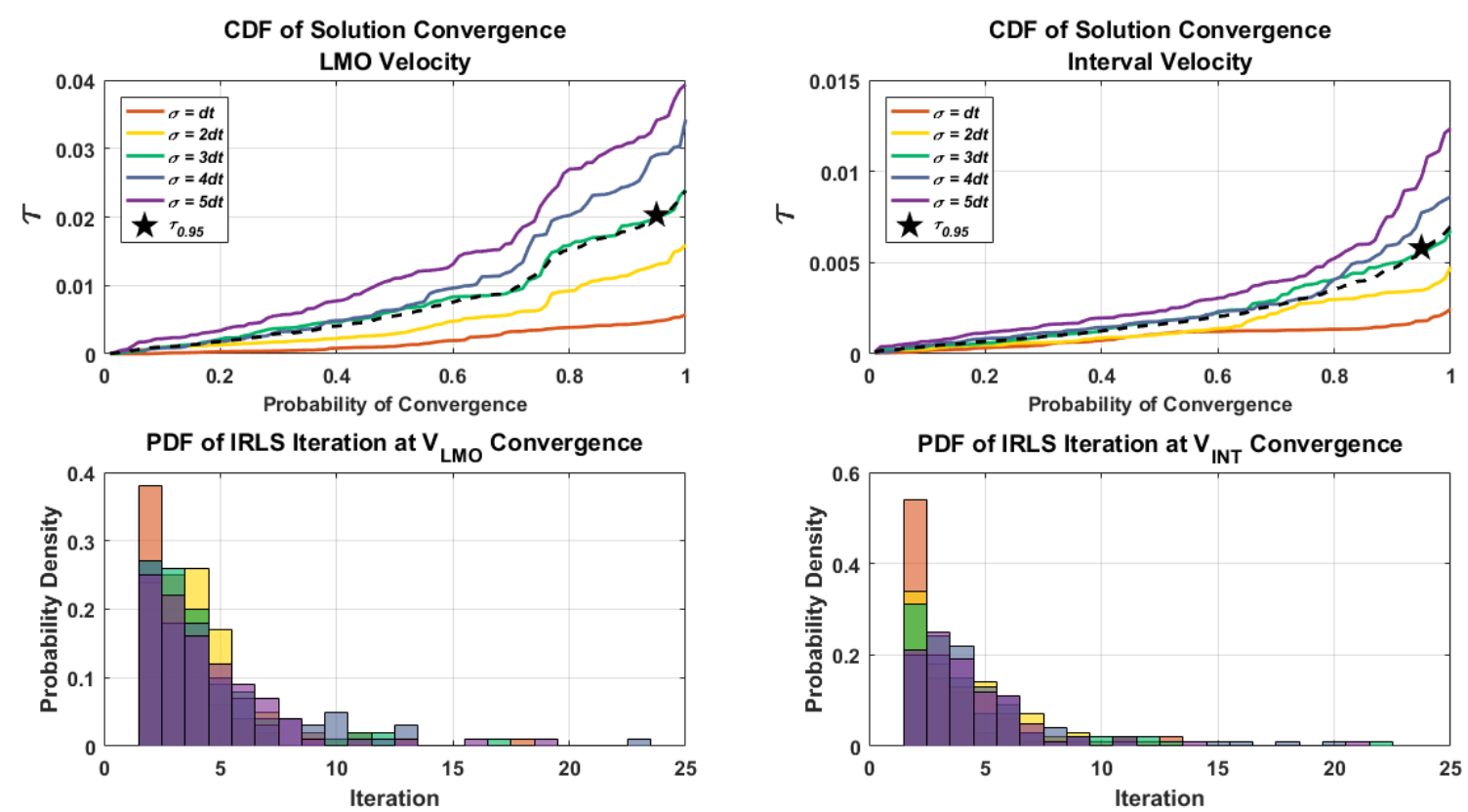

Figure 2.6: The cumulative distribution function and probability density function for the experimental determination of $\tau$. The simulations were conducted at 5 noise levels $\sigma=[1: 5] \mathrm{dt}$. The PDFs indicate that for any noise level the solution converged within 25 iterations. The dashed black curves on the CDFs represent the ensemble mean. The black star is the determined value for $\tau$; 0.02 for the LMO velocity model and 0.0055 for the interval velocity model. 


\subsubsection{Monte Carlo Bootstrapping for Velocity Uncertainty}

To ascertain the likelihood of the velocity solution estimated by the IRLS $\mathbf{L}_{\mathbf{1}}$ minimized regression I leverage statistical computation to bound the estimated parameters with confidence intervals. I implement a bootstrapping algorithm of the examined shot gathers to estimate statistics of the velocity regression. Bootstrapping is a Monte Carlo approach that approximates a normal distribution of the regression parameter. The bootstrapping strategy follows the central-limit theorem and becomes widely applicable in problems where the underlying statistical theory of the parameter distribution is unknown. By randomly sampling the travel-time observations with replacement, the velocity solution is replicated many times with varying perturbation.

Travel-time picks of a consistent phase horizon across all radar channels make the sample population. For the multi-channel radar velocity regression, this is a small population of no more than 9 data points for a single shot record. In my bootstrapping algorithm I expand the possible solution perturbation by randomly decimating the quantity of channels provided to the velocity regression. I allow 0,1 , or 2 channels, chosen at random, to be cut - owning 100, 89, or $78 \%$ of full-fold data. The resampled travel-time observations estimate the two parameters of the velocity regression by the jackknife technique (Efron \& Stein, 1981). One shot record in this way will be exhausted of possible outcomes after 46 unique draws. To further expand the population and incorporate the spatial variability of the snow and firn, I group the 10 nearest neighboring shot gathers into the sample pool. The spatial averaging of this approach is $\sim 22.5 \mathrm{~m}$ or approximately twice the length of the antenna array. I ensure that a single antenna separation is not represented more than once for any 
bootstrapped realization. Efron \& Tibshirani (1986) remark 1000 realizations are adequate for ratifying a distribution which can establish confidence intervals. I adhere to the guideline by drawing 1000 samples at random from a permutation which generates greater than 1 million outcomes. In a multi-channel seismic study, Sacchi (1998) performed bootstrapping during spectral velocity analysis to improve the resolution of the coherency measure and achieve the standard error of the NMO analysis. The chief complaint of this method is computational cost; the spectral velocity method is costly without the repetition of Monte Carlo. Bootstrapping is feasibly conducted during horizon velocity analysis because the regression targets specific travel times on a user identified, coherent horizon.

\subsubsection{Confidence Intervals}

The bootstrap distribution $\hat{G}$ is generated from 1000 jackknifed realizations of the travel-time observations. A distribution is gathered for each parameter - zero-offset intercept travel-time and rms velocity. The bootstrap method is useful for assessing solution confidence because the parameter distributions can be expressed by a mean and a variance. The mean of $\hat{G}$ yields the expected value of the parameter $(\hat{\theta})$ that has a standard deviation $(\hat{\sigma})$. Efron \& Tibshirani (1986) show that confidence intervals for a bootstrapped distribution can be calculated using the assumption of a standard normal distribution (equation 2.22).

$$
\theta_{S}[\alpha]=\hat{\theta}+\hat{\sigma} \hat{z}^{(\alpha)}
$$

The z-score $(z)$ assessed at a confidence level $(\alpha)$ is a scalar that establishes the solution bounds of the parameter mean $(\hat{\theta})$. I choose the confidence interval $(\mathrm{CI})$ at the $\alpha=0.15865$ level that yields the approximate central interval $1-2 \alpha=68.27 \%$. 
The EM velocity and travel-time uncertainties flow through the snow accumulation product of the multi-channel radar. The estimation and appraisal of velocity-derived snow and firn density, depth, and accumulation are discussed in Section 2.5.

\subsection{Coupling Electromagnetic Velocity and Polar Snow and Firn}

The rigorous $\mathbf{L}_{\mathbf{1}}$ regression with bootstrapping, estimates the rms velocity of the radio wave moveout and provides the means to invert this solution for an estimate of the physical mass of the snow and firn. I consider these estimates to be continuous because the shot or CMP interval is dense compared to the length of radar transects; rather than sparse CMP sampling which have intervals on the tens-kilometer scale as in the work by Brown et al. (2012). The continuous acquisition strategy allows for estimates which capture subtle to large changes in the snow accumulation and firn density gradients. The variation in accumulation and density occurs within the pixel of downscaled regional climate models as shown later in this work (Chapter 3).

Within this section I elaborate on the dielectric mixing formula used for the physical quantification of snow and firn density. I discuss the uncertainty appraisal of the density and depth estimates. I then provide the accumulation product and discuss the propagation of error through this calculation.

\subsubsection{Complex Refractive Index Method for Estimating Snow and Firn Density}

"The densification of snow is ... a powder transformed into a solid mass by eliminating pore space and reducing surface area" (Anderson \& Benson, 1963, p. 392). During the cold season, albeit recent discoveries of multi-annual englacial aquifers 
(Forster et al., 2014), polar snow and firn is a monomineralic formation composed of ice with the pore space occupied by air. When examining a sample of dry snow (prior to the onset of melt) or cold firn from within ice sheet, the physical constituency of this dielectric media becomes clear. In short, a dielectric medium is an electrical insulator that becomes polarized when subjected to an electric field. The snow and firn may be represented as a mixture of the dielectric properties of ice and air. The electrically resistive properties of ice and air allow radar measurements to perform exceedingly well in this environment.

The relative dielectric permittivity $\left(\epsilon_{r}\right)$ and relative magnetic permeability $\left(\mu_{r}\right)$ are unitless quantities. Where $\epsilon_{0}$ and $\mu_{0}$ are the absolute quantities in free-space and $\epsilon_{k}$ and $\mu_{k}$ are the absolute quantities within matter $(k)$, the relative dielectric permittivity (2.23) and relative magnetic permeability (2.24) are expressed as a ratio.

$$
\begin{aligned}
\epsilon_{r} & =\frac{\epsilon_{k}}{\epsilon_{0}} \\
\mu_{r} & =\frac{\mu_{k}}{\mu_{0}}
\end{aligned}
$$

The following computations apply the relative quantities and are given the subscript a, i, or s/f, for air, ice, or snow/firn, respectively. The relative dielectric permittivity of pure ice containing no vesicles of air is $\epsilon_{i}=3.15$ (Ulaby et al., 1986). The density of pure ice is $\rho_{i}=917 \mathrm{~kg} / \mathrm{m}^{3}$ (Herron \& Langway, 1980). Known $\epsilon_{i}$ and imposing $\mu_{i}=1$, the electromagnetic velocity of pure ice can be estimated (2.25).

$$
V_{i}=\frac{V_{a}}{\sqrt{\epsilon_{i} \mu_{i}}}
$$


I consider snow and firn pore space to be weightless, have the relative dielectric permittivity $\epsilon_{a}=1$, and moveout with the velocity $V_{a}=0.2998 \mathrm{~m} / \mathrm{ns}$. The less significant digits of EM velocity are immeasurable by the multi-channel radar technique and have been dropped. $V_{i}$ is $0.1689 \mathrm{~m} / \mathrm{ns}$ for the relative dielectric constant $\epsilon_{i}=3.15$. Fowles (1975) provides a holistic review of optical physics including the experimental determination of the speed of light and radio waves traveling within a vacuum and other media.

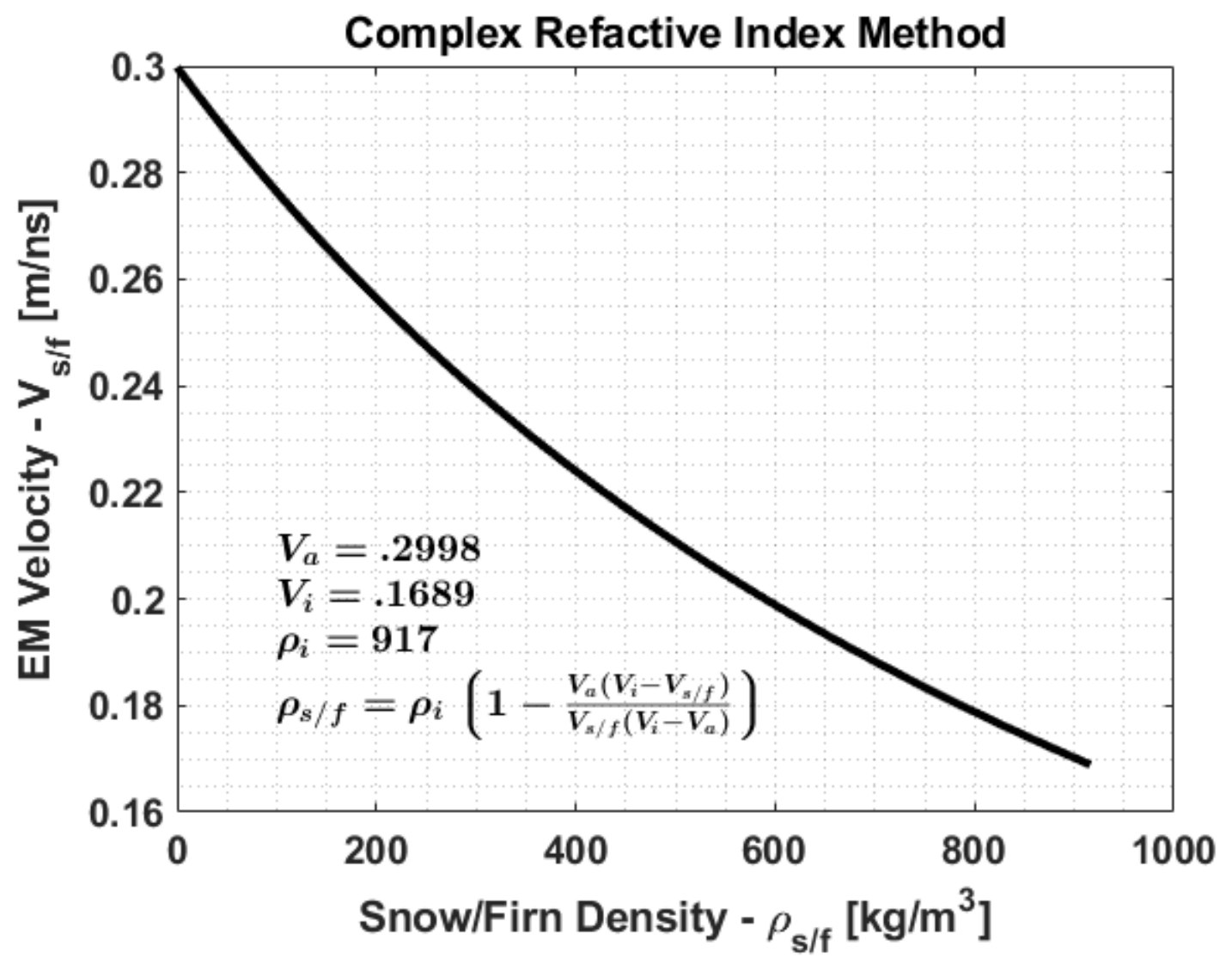

Figure 2.7: The two-phase CRIM model applied to dry snow/firn. Constants used and the CRIM equation are within the figure. 
The index of refraction $(n)$ is the ratio of the velocity of EM radiation in a vacuum to that in matter. By rearranging of equation (2.25) and substituting the known constants for unitless the relative dielectric permittivity and relative magnetic permeability the refractive index of ice is calculated (2.26).

$$
n=\frac{V_{a}}{V_{i}}=\sqrt{\epsilon_{i} \mu_{i}}=\sqrt{3.15}=1.775
$$

The complex refractive index method (Rogriguez \& Abreu, 1990) is a non-linear formula that relates known dielectric properties to an unknown bulk property. Rogriguez \& Abreu's (1990) formulation of the CRIM equation shows how $n$, the refractive index, simplifies the algebra of the CRIM expression. I formulate a two-phase dielectric mixing model of ice and air. Liquid water within the firn column was not present during the field study and is not considered in the CRIM model. I arrive at equation (2.27) for $\rho_{s / f}$, the snow or firn density.

$$
\rho_{s / f}=\rho_{i}\left(1-\frac{V_{a}\left(V_{i}-V_{s / f}\right)}{V_{s / f}\left(V_{i}-V_{a}\right)}\right)
$$

$V_{s / f}$ is the estimated EM velocity of the snow or firn. If the rms velocity is supplied into the CRIM equation the average density will be output. Likewise, if the interval velocity is estimated through Dix's equation (D.15) and supplied in to the CRIM model, the interval density will be output. Figure 2.7 displays the curve which is used to map EM velocity to snow or firn density.

\subsubsection{Jackknife Appraisal of Density and Depth}

Following the bootstrapping approach for estimating the rms velocity of the snow or firn and zero offset travel-time to the coherent horizon, I have created bootstrap 
distributions of density and depth. Each replication of the velocity estimate is modeled by the CRIM equation (2.27) to simulate many jackknifed replications of the density. Similarly, the intercept travel-time replications are applied in conjunction with the velocity estimate to simulate replications of the horizon depth via equation (D.18). During this process the interval densities and thicknesses may also be appraised following Dix's solution (Appendix D.3). Confidence intervals for depth and density are assessed via the standard normal distribution at $68.27 \%$ or $1 \sigma$ (Section 2.4.10). The sample variance (equations 2.28 and 2.29) and covariance (equation 2.30) of the density and depth are propagated through the accumulation product (equation 2.33). $\hat{\theta}(i)$ is the $i^{\text {th }}$ jackknife replication of the regression parameter and $\hat{\theta}(\cdot)$ represents the mean parameter of the bootstrapped distribution.

$$
\begin{aligned}
s_{\hat{\rho}}^{2} & =\frac{1}{N-1} \sum_{i=1}^{N}(\hat{\rho}(\cdot)-\hat{\rho}(i))^{2} \\
s_{\hat{z}}^{2} & =\frac{1}{N-1} \sum_{i=1}^{N}(\hat{z}(\cdot)-\hat{z}(i))^{2} \\
s_{\hat{\rho} \hat{z}} & =\frac{1}{N-1} \sum_{i=1}^{N}(\hat{\rho}(\cdot)-\hat{\rho}(i))(\hat{z}(\cdot)-\hat{z}(i))
\end{aligned}
$$

\subsubsection{An Empirical Model Prediction of Density at Depth}

Herron \& Langway (1980) developed an empirical model of dry snow and firn density at depth. The authors tuned the parameters of their model to a handful of firn cores drilled in Greenland and Antarctica during the 1960s-1970s. Their model requires initial conditions for the surface density, the mean annual temperature, and the mean annual snow accumulation. To the first order, the surface density condition controls the density prediction at depth. The mean annual temperature and accu- 
mulation are secondary conditions which guide the density gradient - the latter is only effective beyond the critical density $\left(550 \mathrm{~kg} / \mathrm{m}^{3}\right)$. While temperature is directly proportional to the density estimate, the accumulation rate is inversely proportional. Within the following Chapter (3.3.2) I discuss the multi-channel radar methodology to inform the depth-density model alongside the resulting depth-density predictions.

\subsubsection{Estimating the Snow Accumulation Contribution of GrIS}

\section{Mass Balance}

Meters of water equivalent (MWE) is the height of water produced by a melted snow or firn column. This standard measurement of snow accumulation is the product of the snow or firn density and depth normalized by the density of water $\left(\rho_{w}\right)(2.31)$.

$$
M W E=\frac{\rho_{s / f} \cdot z}{\rho_{w}}
$$

The average accumulation above a given reflection horizon is estimated through the rms velocity. Intervals of the firn bounded by horizon picks yield estimates of the interval accumulation when this process is supplied the interval velocity. Stratigraphic horizons bounded with ages $A_{n}$ can deliver the accumulation rate estimate (2.32).

$$
\frac{d M W E}{d t}=\frac{M W E}{\left(A_{1}-A_{2}\right)}
$$

$A_{1}$ is the earlier annual horizon, $A_{2}$ is the later. Multi-channel radar velocity analysis provides the avenue for mapping the firn stratigraphy at depth. This tool can be used between GreenTrACS core sites, where firn depth-age profiles exist, to extrapolate the spatio-temporal variability of Western Greenland snow accumulation. 


\subsubsection{Appraisal of the Accumulation Estimate}

Symmetric confidence intervals bound the accumulation estimate at the $\alpha=$ 0.15865 level. The variance of MWE is estimated by the linear error propagation equation (2.33).

$$
s_{M W E}^{2}=s_{\hat{z}}^{2} \hat{\rho}(\cdot)^{2}+s_{\hat{\rho}}^{2} \hat{z}(\cdot)^{2}+2 s_{\hat{\rho} \hat{z}} \hat{\rho}(\cdot) \hat{z}(\cdot)
$$

The resulting uncertainty measure is the standard interval,

$$
M W E_{S}[\alpha]=M \hat{W} E+s_{M W E} z^{(\alpha)}
$$

where $z^{(0.15865)}=1$. Bootstrapping is effective for drawing sample variance and covariance estimates of density $(\rho)$ and depth $(z)$. By propagating the variances of density and depth through equation (2.33) the covariance term of accumulation is incorporated. I expect depth and density to vary in a harmonious direction; as depth increases so does the density. The subtle variances that occur in the annual surface of GrIS are not as strongly correlated in this way. Wind driven densification can significantly alter the density of a shallow annual snow. The results of the multichannel radar analysis are presented in the subsequent chapter (3). Therein, I discuss the effectiveness of the methods enclosed within this chapter and provide some tutorial on identification of the air wave, the surface wave and the initial reflection. 


\title{
CHAPTER 3:
}

\section{RESULTS: MULTI-CHANNEL RADAR DATA}

\begin{abstract}
ANALYSIS
The MxRadar was towed from the Core Site 15 south and west to the Western Spur Snow Pit along a $45 \mathrm{~km}$ segment of the 2017 GreenTrACS route (Figure 1.1). Surface elevation rises $\sim 20$ masl during the $15 \mathrm{~km}$ southward segment, then falls $\sim$ 100 masl throughout the $30 \mathrm{~km}$ westward segment (Figure 3.1). Estimated snow and firn densities have a negative deviation as surface elevation increases and a positive deviation as surface elevation decreases (Figure 3.9). The MxRadar results agree with the two points of evaluation: Core 15 and 15 W Snow Pit. Radiostratigraphic imaging depicts laterally continuous IRHs to $\sim 22.5 \mathrm{~m}$ depth that approximately span the period 2000 - 2017 CE. A bright reflection horizon correlates with the change in densification regime described by Anderson \& Benson (1963) at the critical depth. Snow accumulation varies spatially along the Core 15 Western Spur (Figure 3.3). Maximum accumulation of $\sim 0.725 \mathrm{~m}$ w.e. $a^{-1}$ occurs in a trough that is $5 \mathrm{~km}$ wide and minimum accumulation of $\sim 0.55 \mathrm{~m}$ w.e. $a^{-1}$ occurs on the flank of the trough. The average accumulation here is estimated at $\sim 0.625 \mathrm{~m}$ w.e. $a^{-1}$ by the MxRadar method.
\end{abstract}




\subsection{GreenTrACS Core 15}

In this chapter, I examine a $\sim 45 \mathrm{~km}$ transect referred to as the Core 15 Western Spur. The Core 15 site is chosen from the 2017 campaign because this location accumulates a large amount annual snowfall $\sim 0.65 m$ w.e. for a high elevation site $\sim 2600$ masl. The core drilled here is $\sim 28.5 \mathrm{~m}$ and extends past the deepest recorded radar echo. $30 \mathrm{~km}$ of the radar transect overlap a repeated Operation IceBridge flight line. These data can provide a direct comparison to or supplement to available remote sensing data. Figure 1.1c is a map of the Core 15 Spur W and Figure 3.1 is the topographic section of the surface elevation.

In Section 3.2, I provide a tutorial on identifying the air wave, the surface wave, and the primary reflected wave. The results of the MxRadar density, depth, and accumulation product are presented in Section 3.3. Additional methods of the data analysis are introduced to join the MxRadar approach with the Herron \& Langway (1980) density model to predict the firn density at depth. The joint approach is evaluated at Core Site 15 and the Western Spur Snow Pit (Section 3.3.2). This method is applied to depth profiling of radiostratigraphy, density, accumulation, and age in Section 3.4. 


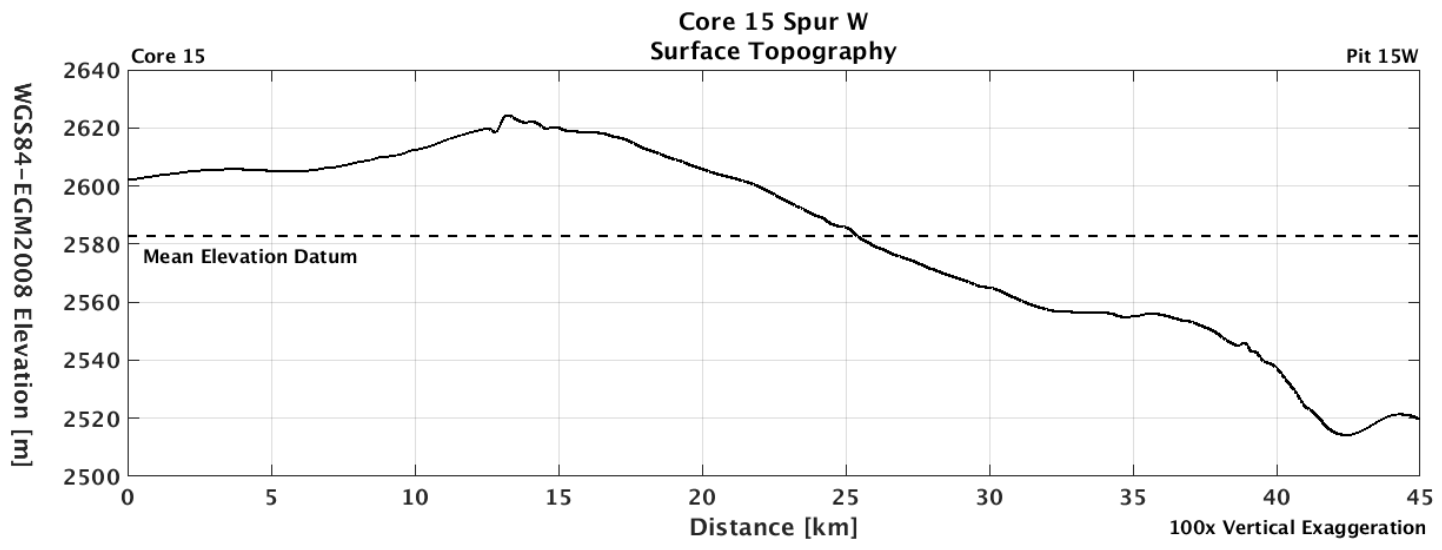

Figure 3.1: The surface elevation above mean sea level for the Core 15 Spur W traverse. Post-processed GPS elevations relative to the WGS84 ellipsoid model have been corrected to the EGM2008 geoid surface to deliver elevations above mean sea level. Notice the trough nearest to the end of the transect. This topographic feature captures greater snow accumulation.

\subsection{Multi-channel Radar Signal Interpretation}

The success of the direct and reflected wave analysis is determined by the quality of the signal and the geophysicist's ability to correctly identify and select the wavefield features. To aide in the identification of the recorded wavefield characteristics, I present annotated radar gathers for offsets $4 \mathrm{~m}, 8 \mathrm{~m}$, and $12 \mathrm{~m}$. The data prepared in Figure 3.2 have been manipulated by the processing presented in Chapter (2.3).

I have overlain and identified my selections of the air-coupled and surface-coupled waves, and the initial reflected energy. I have chosen the central peak of the Ricker wavelet pulse as the matched phase. The wavelet peak becomes the reference datum for travel-time interpretation after the time-zero correction is applied (Chapter 2.4.2). At the farthest offset $(12 \mathrm{~m})$ the initial trough of the air wave is indistinct and presumably lost within the noise floor of the instrument. I have applied AGC within 
a 50 sample window to clearly display air-wave that is weakly radiated because of the antenna shielding. An effect of frequency domain filtering is identified on the top of the sections. This striping effect and the instrument noise character are not apparent without AGC.

Lateral waves contaminate the signal and make identification of the important waveforms difficult. I interpret these waves to be refracted and laterally moving because this energy appears on the time record prior to reflected waves. For near-offset gathers $(1.33 m-4 m)$ lateral waves dominate the initial reflection signal. The cycles of these lower amplitude lateral waves dissipate as the horizontal offset increases, by interference between the laterally bound surface wave and upgoing reflected wave. Matching the phase of the initial reflection requires careful tracking of the reduction in lateral wave cycles. Two prominent lateral wave peaks are evident in the $4 \mathrm{~m}$ gather. At $8 \mathrm{~m}$ offset, the initial peak is nearly extinguished, while only one peak is evident on the farthest $(12 \mathrm{~m})$ gather prior to the reflected wavelet. Free-surface lateral waves are prevalent on the mid to far-offset gathers $(5.33 m-12 m)$. These waves are subsequent to the direct air arrival and are caused by upgoing energy that is critically refracted at the snow/air boundary. Refracted air-coupled waves are distinguished by lower amplitude and decreased waveform consistency. Critically refracted energy is masked by the AGC shadow zone in the mid-offset range, due to the proximity of the the air-coupled arrival to the large amplitude surface-coupled wave. Within Appendix D.5, I determined the arrival times of radio energy following a critically refracted raypath through a homogeneous snow pack model. The results of this experiment corroborate the presence of the lateral wave features identified in Figure 3.2. 

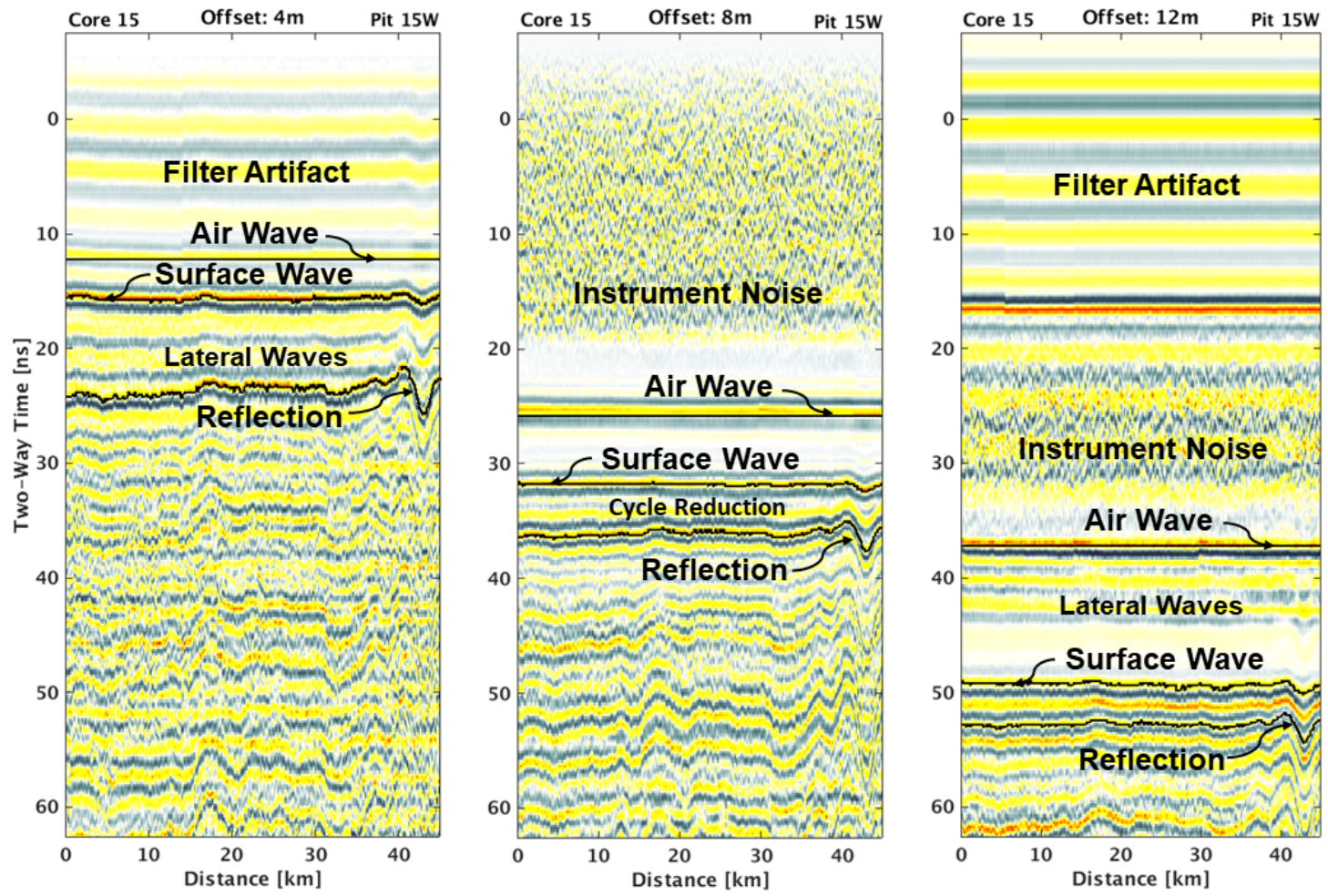

Figure 3.2: Offset gathers $4 \mathrm{~m}, 8 \mathrm{~m}$, and $12 \mathrm{~m}$ for the traversed route from Core 15 to the spur's end are annotated to identify the important wavefield features. The peak amplitudes of the picked horizons are blotted out in black and are identified with arrows. It is important when matching the initial reflection horizon phase across offset gathers to account for the reduction in cycles of the lateral waves bounded by the surface and reflection waves. 


\subsection{Surface Density, Depth, and Accumulation}

I performed the horizon velocity analysis (Chapter 2.4) on the three selected horizons diagrammed in Figure 3.2. The snow/firn velocities are converted to an estimate of density via the CRIM equation (Chapter 2.5). This analysis yields an estimate of the near-surface density, the depth to the reflection horizon, and the accumulation of snow mass to this depth. Figure 3.3 presents the 2016-2017 accumulation, the average densities of the surface-coupled wave and the reflected wave, and the depth of the annual horizon. Uncertainties in each of these estimates are expressed at the $1 \sigma$ confidence level. The methods used for the uncertainty analysis are detailed within Chapters 2.5.2 and 2.5.5. The output has been convolved with a 251 point hamming window (equation C.7).

\subsubsection{Corrections for Instrument and Horizon Depth Biases}

When evaluated at the Core 15 and Pit 15W sites, the multi-channel radar measure of stacking velocity maintained a positive bias of $0.004 \mathrm{~m} / \mathrm{ns}$ greater than observed in the core and snow pit, after converting density measurements to EM velocity. This estimate is within the velocity error of the instrumentation documented by Bradford \& Harper $(2005)$ to be $\pm 0.005 \mathrm{~m} / \mathrm{ns}$. For estimates of the snow surface velocity on this transect the error is less than $2 \%$. For each velocity estimate along the Core 15 western spur I have subtracted $0.004 \mathrm{~m} / \mathrm{ns}$ from the horizon velocity analysis result.

The annual horizon depth must also be corrected for bias, because the reflected energy has returned from a stratum $\sim 15 \mathrm{~cm}$ deeper than the soft hoar layer identified as the annual surface in Pit 15W. $\sim 15 \mathrm{~cm}$ is subtracted from the reflection horizon depth estimated during velocity analysis. This correction is less than $10 \%$ at Pit $15 \mathrm{~W}$ and represents $0.0565 \mathrm{~m}$ w.e. 


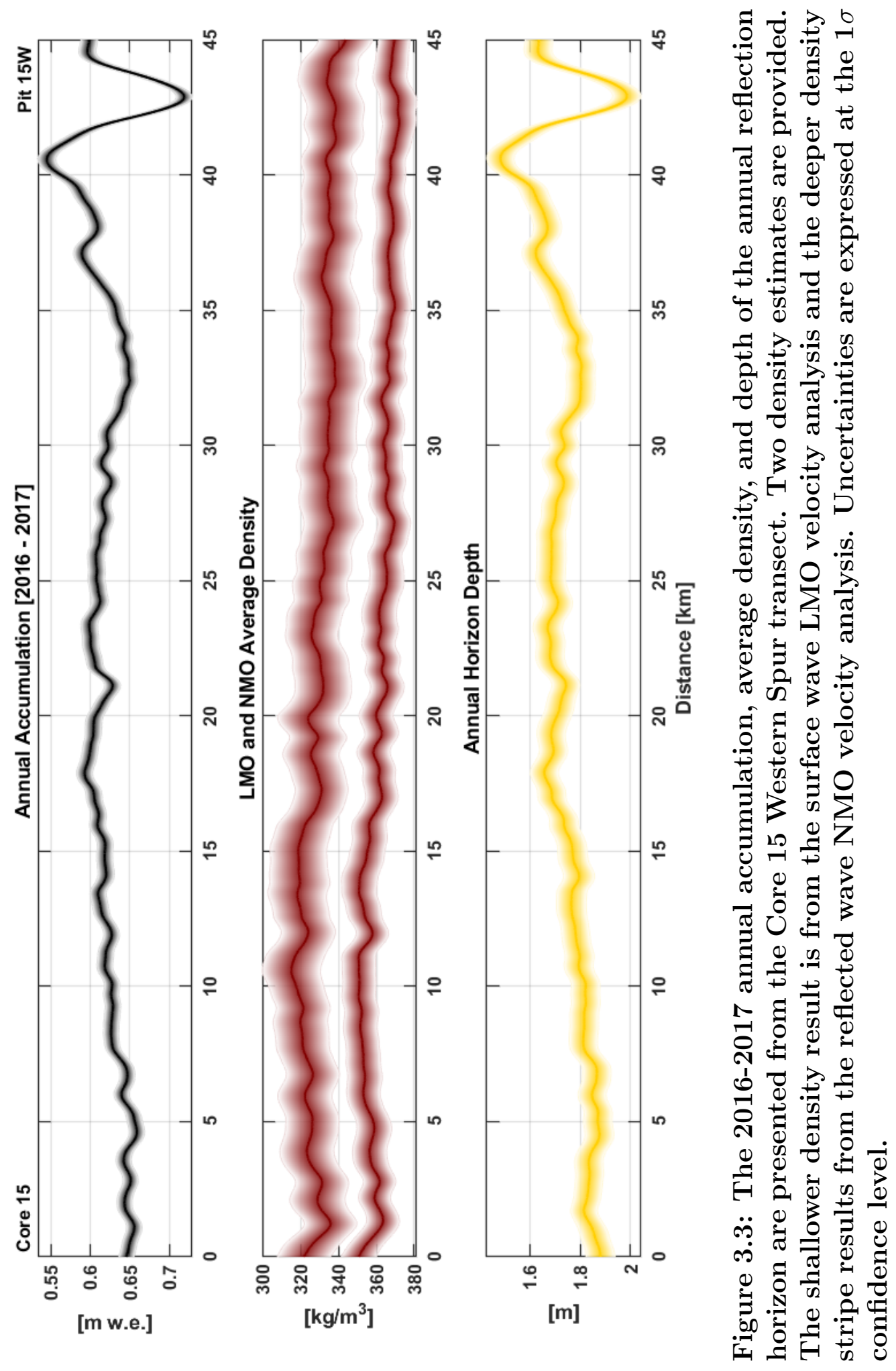




\subsubsection{Radar Forcing to Extrapolate Density at Depth}

Picking travel-time horizons much beyond the initial reflection becomes cumbersome for the many offset gathers. Stacking velocity estimates from later travel-time picks display inaccuracies of the type shown in the synthetic case for $\sigma=5 d t$ (Figures D.2b and D.5a). To extrapolate a stacking velocity model, the physical information of the ice sheet surface gained from the multi-channel strategy is used to force the parameters of the Herron \& Langway (1980) firn compaction model (Chapter 2.5.3); dubbed, the MxRadar Herron Langway (MxHL) snow and firn compaction model. The MxHL model accurately estimates the $0-10 \mathrm{~cm}$ surface density and density at depth when evaluated at the Core 15 and Pit $15 \mathrm{~W}$ sites.

A few additional steps of data analysis are required to extrapolate the $0-10 \mathrm{~cm}$ surface density and the density model prediction. The number system $(\mathbf{1}-\mathbf{4})$ is ordered by increasing depth and corresponds to the radar derived estimates and MxHL model forcings (yellow ellipses) in Figures 3.4, 3.5, and 3.6. The average depth and density of the direct surface-coupled wave (2) and the initial reflected wave (4), which is the "commonly reported average density over the first one or two meters of snow" (Herron \& Langway, 1980, p. 7), informs the density parameter (3) of the model. The surface intercept density (1) is directly solved by the linear regression Monte-Carlo bootstrapping approach (Chapter 2.4.9), using the density estimates of the surface wave (2) and initial reflection (4).

A least-squares fit is drawn through the model forcings $(\mathbf{1}-\mathbf{4})$, and I join this linear piece to the Herron \& Langway (1980) model at the depth of forcing (3). By doing so, I make an important improvement to the empirical model which accounts for the more spatially variable snow densification process. The MxRadar approach 
calculates the rate of snow densification with depth and is sensitive to inversion in the average snow density profile.

At Core Site 15, I tested integer values for mean annual air temperature between $-25^{\circ} \mathrm{C}$ and $-15^{\circ} \mathrm{C}$ as input for the temperature forcing of the MxHL model. The RMSE (equation D.25) between the extracted core and the modeled density was minimized at $-19^{\circ} \mathrm{C}$, when forced by the radar derived annual accumulation and density. I compare the MxHL model to the Core 15 density log in Figure 3.4, and the cumulative averaged density profile - analogous to the stacking velocity model - in Figure 3.5. Uncertainties for the Core 15 data are fabricated by a 3point running variance calculation; uncertainties are expressed at the $1 \sigma$ level for all data plotted. The MxHL model agrees with the Core 15 profile reasonably well: $R M S E=41.3 \mathrm{~kg} / \mathrm{m}^{3}$. The integral average density of the MxHL model expresses a stronger fit: $R M S E=7.3 \mathrm{~kg} / \mathrm{m}^{3}$. Recall, the radar velocity estimate is sensitive to the integral average density. Resolving the density profile with the multi-channel radar will always entail some amount of vertical and lateral averaging. Local variability in Core 15 explains much of the disagreement between the model and core log, as the MxHL model is not sensitive to fluctuations of the firn density. The following section (3.4) reviews the radar product - depth imaging of snow and firn stratigraphy, density, and age. 


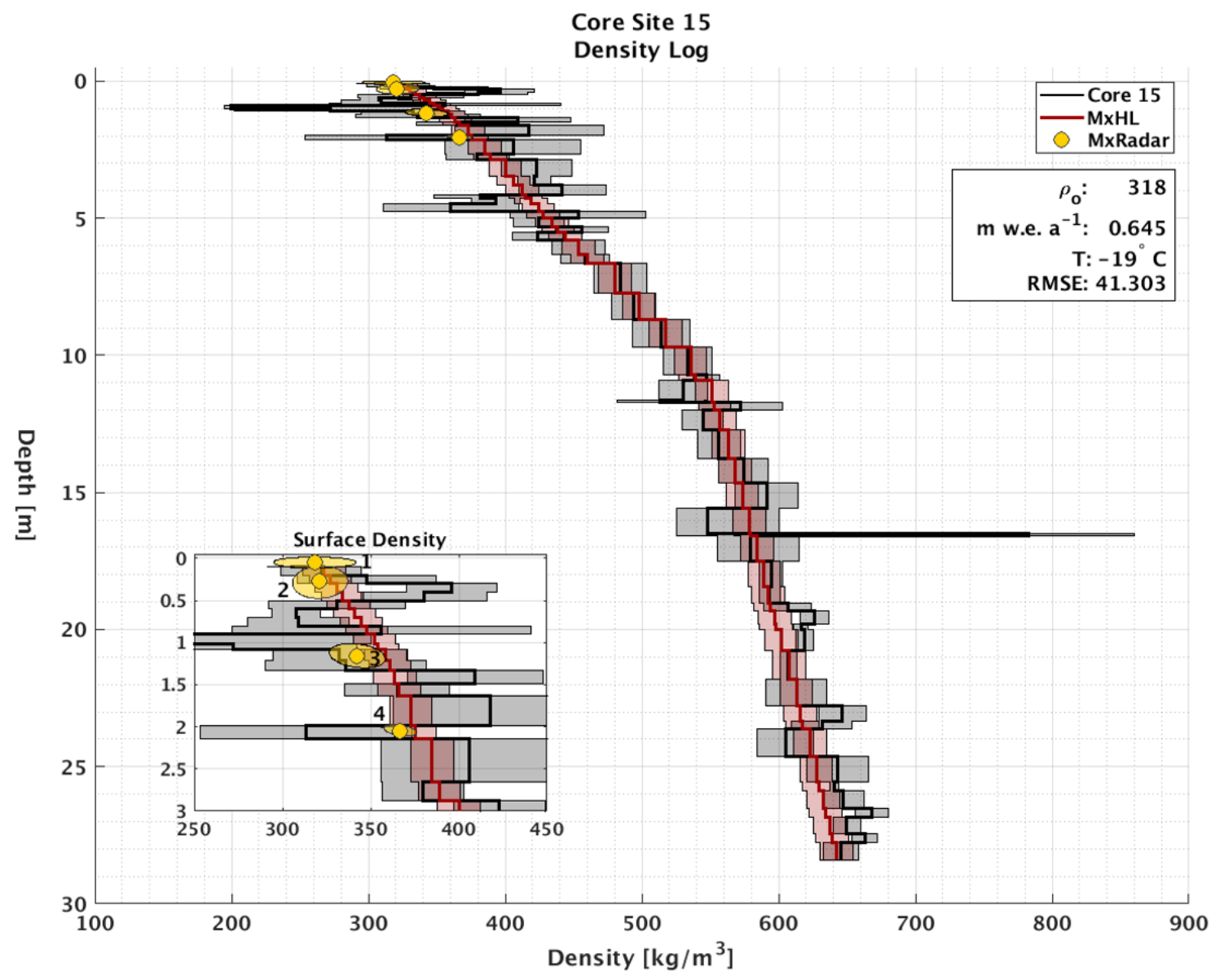

Figure 3.4: Plotted in black is the density log of GreenTrACS Core Site 15. The Herron-Langway model, plotted in red, estimates at depths matching those of core segments. In the lower left, the upper $3 \mathrm{~m}$ is enlarged to show the surface density clearly. Yellow ellipses display the multi-channel radar estimates that drive the density model. Numbered: (1) the surface density, (2) the LMO density, (3) the forcing density for the Herron \& Langway (1980) model segments, (4) the NMO density. Uncertainties are expressed at the $1 \sigma$ level. The RMSE $=41.3 \mathrm{~kg} / \mathrm{m}^{3}$ between the MxHL model and core log. Much of the misfit can be explained by the Core 15 variance. 


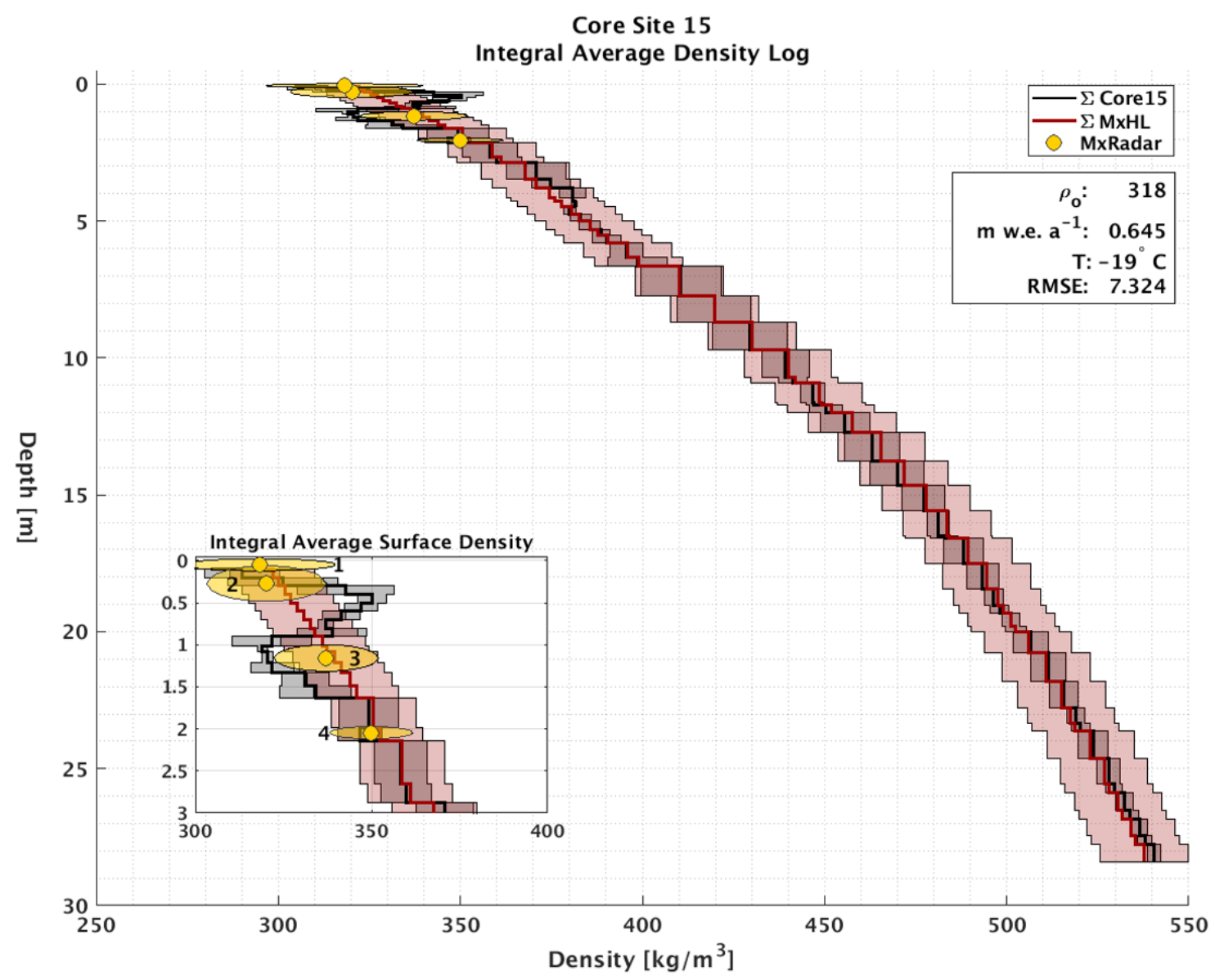

Figure 3.5: A cumulative average has been calculated for the Core Site 15 density $\log$ and the MxHL model. In the lower left, the upper $3 \mathrm{~m}$ is enlarged to show the integral averaged surface density clearly. The MxHL snow and firn compaction model is driven in the integrally averaged domain. A good fit exists RMSE $=7.3 \mathrm{~kg} / \mathrm{m}^{3}$. Variation of the Core 15 density log is removed by the vertical averaging. Uncertainty is measured at the $1 \sigma$ confidence level. 

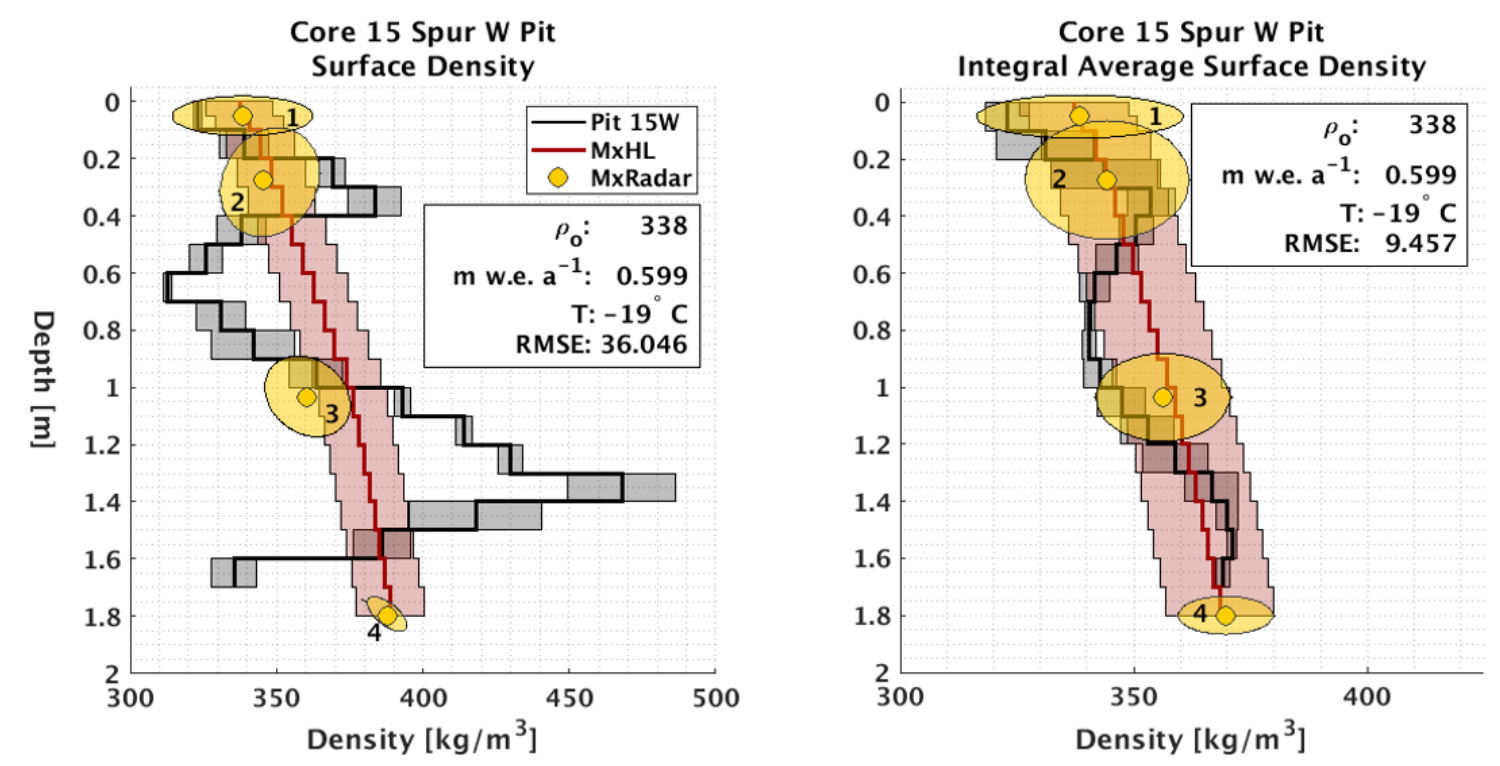

Figure 3.6: The multi-channel radar derived result is first evaluated at Pit 15W, corrected for bias, and then evaluated at the Core 15 site. This figure presents the bias corrected result with the radar information (yellow ellipses) used to inform the MxHL snow and firn compaction model. The integral averaged density is sensed by the radar and Dix's conversion is used to retrieve the interval density. Due to wavelength limited resolution of the radar, the estimated density profile is a simple linear approximation of the snow density.

The methodology is evaluated at the Pit 15W location in Figure 3.6. A good agreement exists between the snow pit density and the MxHL model density: RMSE = 36.0 and 9.5 for interval density and integral density respectively. A positive change in surface density between Pit $15 \mathrm{~W}$ and Core Site $15\left(\Delta \rho_{0}=20 \mathrm{~kg} / \mathrm{m}^{3}\right)$ is observed and a negative change in accumulation $\left(\Delta A a^{-1}=-0.045 m w . e . a^{-1}\right)$ is predicted. Yellow ellipses are numbered in Figures 3.4-3.6: (1) the surface density, (2) the LMO density, (3) the forcing density for the Herron \& Langway (1980) model segments, (4) the NMO density. 


\subsection{Depth Imaging of Snow and Firn: Stratigraphy, Density, and Age}

The Core 15 Western Spur traverses across a typically dry-snow zone which has endured surface melt and refreeze within the past half-decade (Nghiem et al., 2012). The extent of surface melt, percolation, and refreeze, here, does not cause the shallow firn density to significantly deviate from the Herron \& Langway (1980) prediction (Figure 3.4). Through this inland region of the GrIS, the MxHL method proves viable as a spatially continuous mapping of the shallow firn density at depth. Within this section I present the depth-converted, stacked radar section, the firn density model, the density deviation from the mean (details the spatial variability of snow and firn compaction), and the age-depth isochronogram contoured with recent years.

\subsubsection{Radiostratigraphy-Depth Imaging}

Following the methodology in Appendix C.6, I present the stacked, depth converted radar section (Figure 3.7). The stratigraphic horizons within the firn of the dry-snow accumulation zone are known to be isochronous - depositional surfaces of a constant age (Jacobel \& Hodge, 1995; Siegert et al., 1998; Eisen et al., 2004; Hawley et al., 2014; Macgregor et al., 2015). Though, the radiostratigraphy does not necessarily represent annual histories from buried melt-refreeze or hoar layers forming during the summer-fall transition, as reported in this work (Section 3.3.1). Mechanical characteristics of the snow/firn density and pore structure give rise to the stratigraphy sensed in the electromagnetic domain (Arcone et al., 2004). The continuous nature of the snow/firn layers allows for large spatial correlation of the stratigraphic depth-age scale (Hawley et al., 2014; Macgregor et al., 2015). 
However, in areas where surface topography dips suddenly the quality of radar echos decays (Arcone et al., 2004). This is apparent at the Core 15 Spur West end where the surface topography troughs and radio echos dip and scatter (Figures 3.1 and 3.7). Few coherent amplitudes persist much deeper than 5 meters, but it is important to track IRHs through this trough, as the accumulation significantly increases here. By modeling the age-depth relationship (Section 3.4.3) and overlaying the isochrons on the radiostratigraphy (Figure 3.11) the continuity of the IRHs is explicated.

\subsubsection{Density-Depth Imaging}

I have applied the MxHL method throughout the Core 15 Western Spur transect to capture the spatial variability of the snow and firn density. Figure 3.8 is a depth section of the spatial density prediction. The density represented is the mean value from the ensemble of possibilities within the $1 \sigma$ confidence interval. The radiostratigraphy is overlain on this figure to obviate the spatially continuous IRHs at all depths. Accumulation can be directly calculated from this digital image by tracking a horizon to extract the density and depth information and applying equation (2.31). The accumulation rate can be estimated between any two horizons using equation (2.32) if the age-depth scale is known.

The density anomaly is represented in Figure 3.9 as deviation from the mean density across a constant depth level. The compaction processes represented by the Herron \& Langway (1980) model and the spatial variability of the surface density and density at depth are evinced. The density model is composed of three segments: snow densification by compaction and grain rounding (upper $0-2 m$ ), the compression driven densification up to the critical density $550 \mathrm{~kg} / \mathrm{m}^{3}$, and the elimination of pore space by molecular diffusion (sintering) of the névé (Anderson \& Benson, 1963). 
The surface density anomaly, depicted in Figure 3.9a, correlates strongly with density anomalies at depth, and differs significantly from the typical values for surface density used in models (personal communication from Jay Zwally, 2017). Predictions of the surface density by the canonical Herron \& Langway (1980) model create a positive bias of $\sim 25 \mathrm{~kg} / \mathrm{m}^{3}$ within the snow pack density due to the tuning of the empirical model. This bias is largest at the snow surface and decreases until the MxRadar segment joins the Herron \& Langway (1980) model at $\sim 1.5 \mathrm{~m}$ depth. Because the MxRadar technique accurately estimates the snow surface density, remotely sensed ice sheet missions for surface mass balance estimation can benefit.

\subsubsection{Age-Depth Imaging}

Theoretical isochrones that abide the steady-state assumption of constant snow accumulation rate are predicted by the Herron \& Langway (1980) model. The 20162017 estimate of annual snow accumulation is propagated through the model to generate the constant time surfaces (Figure 3.10). The increasing plunge with depth of isochronal synclines near Pit $15 \mathrm{~W}$ agrees with the radiostratigraphy - which is clear to the estimated 2014 horizon (Figure 3.11). Plunging isochrones are attributed to spatially variable snow accumulation and propagate through the firn as a kinematic wave, rather than a radar imaging phenomenon ( $\mathrm{Ng} \& \mathrm{King}, 2011)$. The large amount of annual snow accumulation (Figure 3.3) in the Core 15 neighborhood $\left(\sim 0.65 m\right.$ w.e. $\left.a^{-1}\right)$ coupled with significant variation in accumulation about the topographic trough $\left(\sim 0.55-0.725 m\right.$ w.e. $\left.a^{-1}\right)$ limits the horizontal continuity of the radar imaging (Arcone et al., 2004). The dated radiostratigraphy helps identify continuous IRHs through the noisy section of the radar image. In the next chapter I

defend the NMO velocity analysis and extend the discussion of the MxRadar results. 


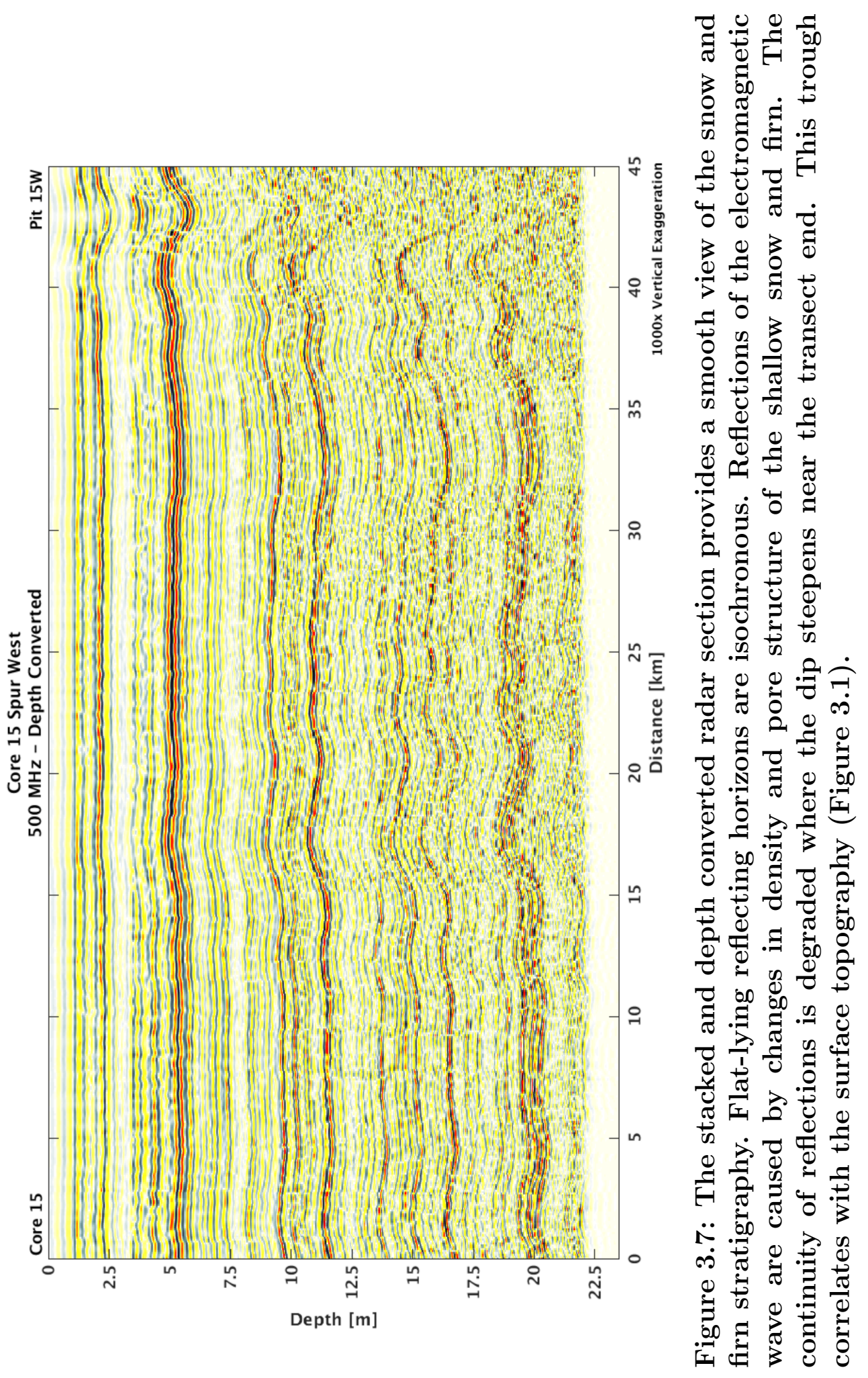




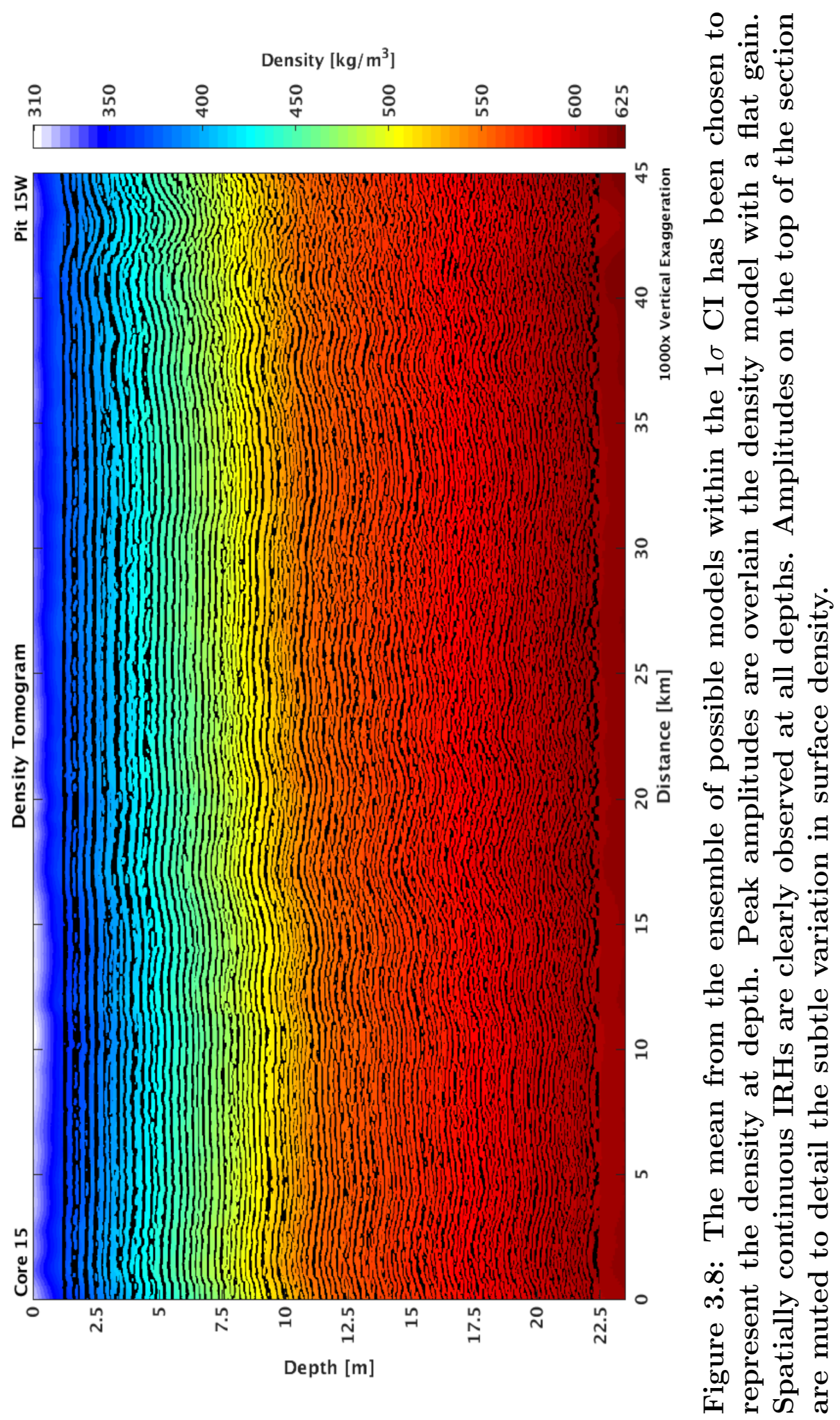




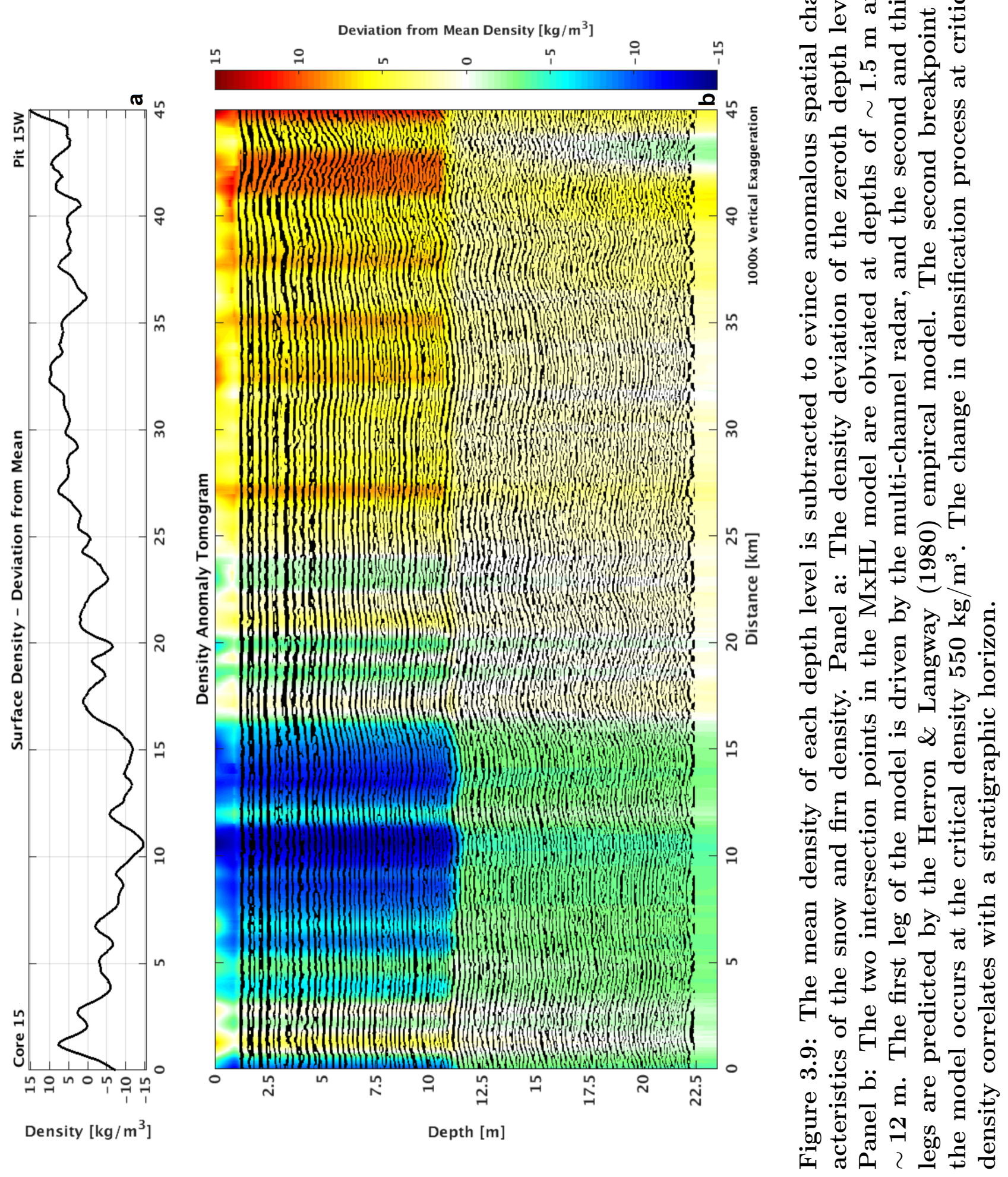




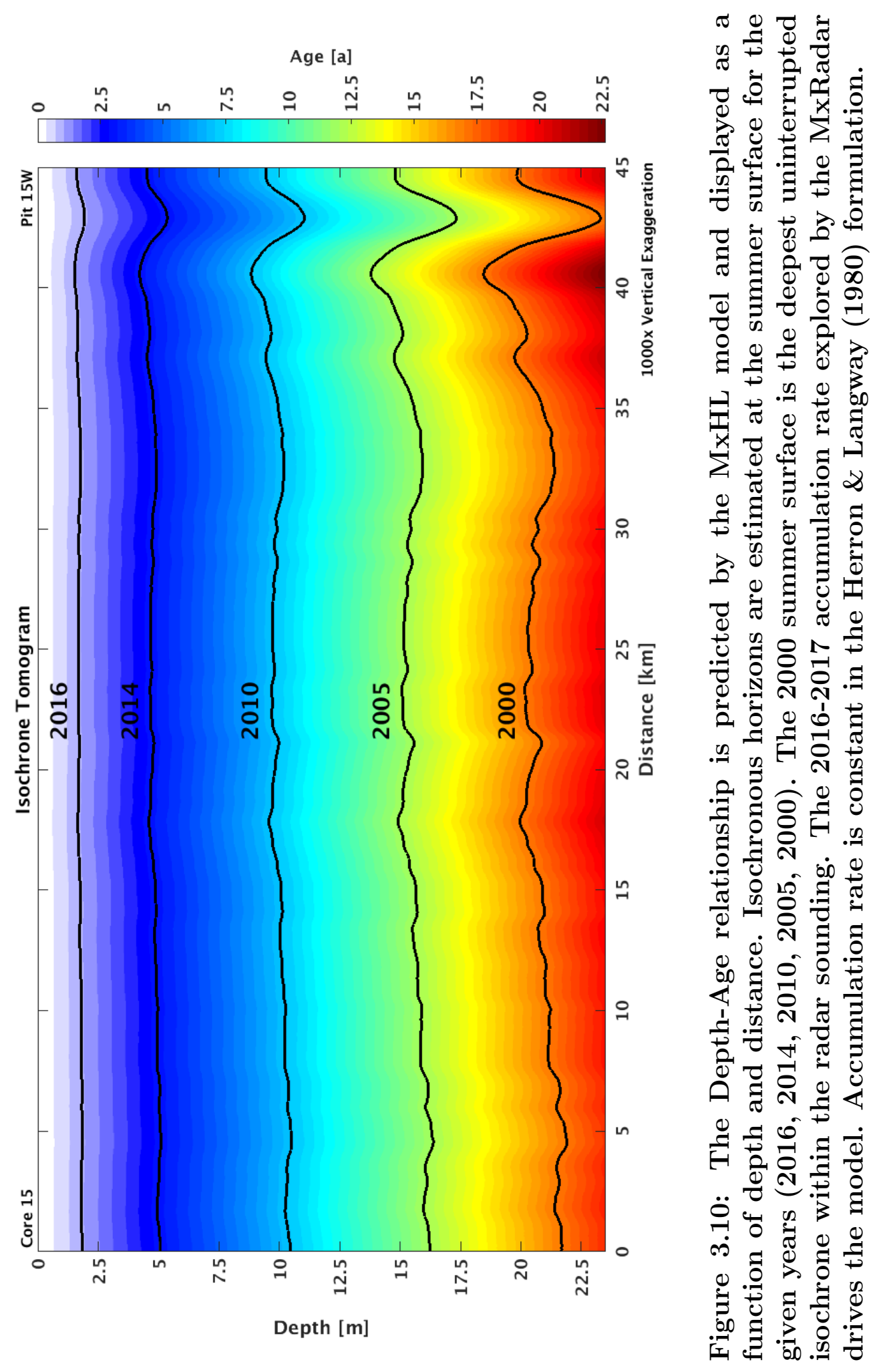




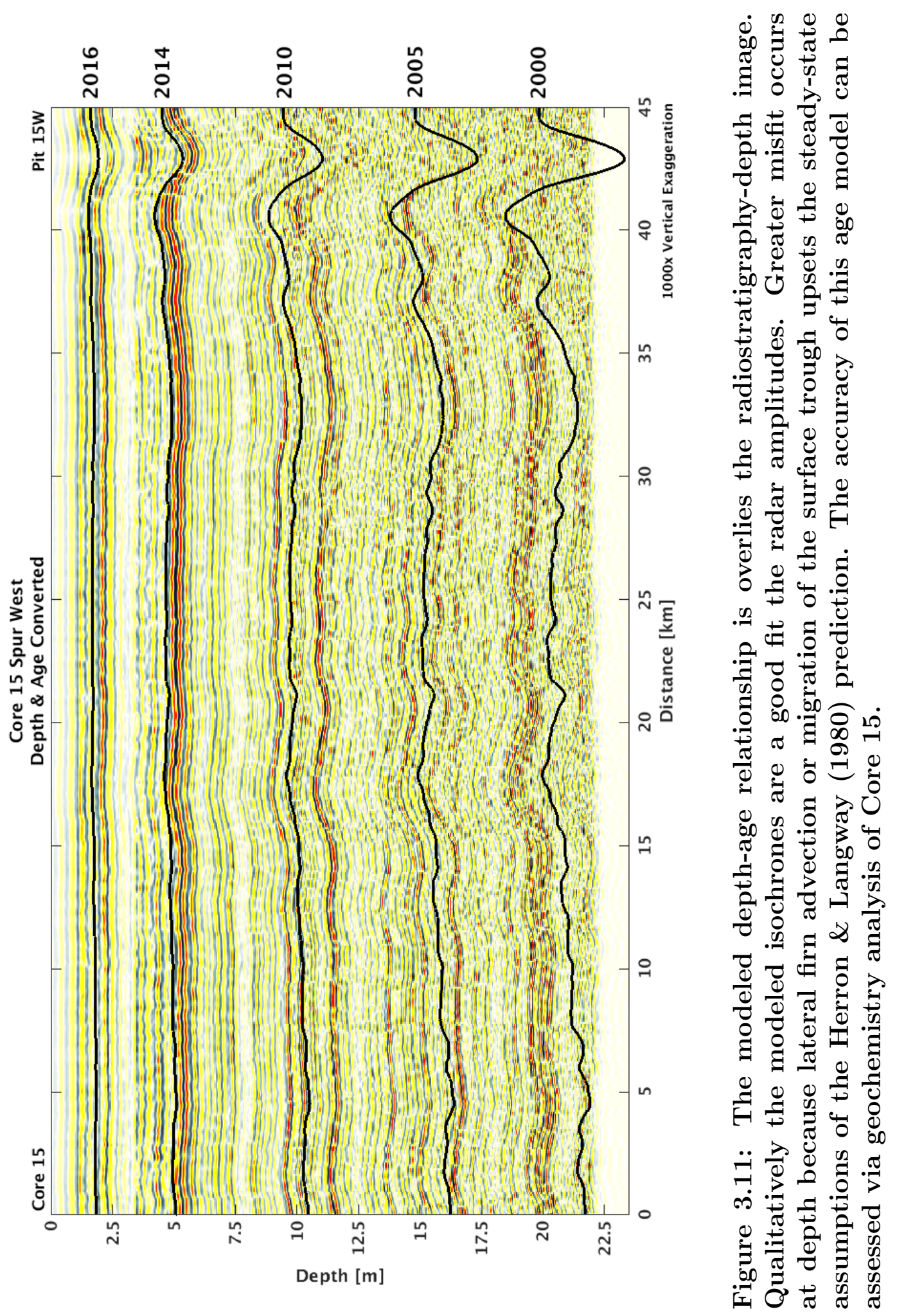




\section{CHAPTER 4:}

\section{DISCUSSION}

I analyzed a typical Greenland ice sheet surface and firn column (hardened, wind packed snow with moderate roughness within a typically dry-snow zone) as a pilot study to ascertain the possibilities and usefulness of a traversed multiple aperture pulsed radar system. Each day of radar collection provides its unique challenges, laboriously - as a technician of radar instruments, physically - as weather conditions evolve surface morphology, and computationally - by algorithmic programming and interpretation of the MxRadar signal. I devoted a large section of this research to developing the traverse acquisition strategy and MATLAB-based software that incorporates the geophysical methods for multi-channel radar signal processing. The

code is published on GitHub and is freely available: https://github.com/tatemeehan/ MxGreenland.

A vast amount of radar data was collected that link the core sites of the GreenTrACS campaign. When tackling a large multi-channel data set, computational efficiency and process automation are required. The direct arrival and primary reflection analysis are engineered to be efficient for both the computer and the geophysicist. The approach is refined for the instance where the Herron \& Langway (1980) model agrees with available firn core data. Additional velocity analysis may be required in regions where the firn profile is densified by meltwater percolation and refreeze, yet the MxHL 
modeled approach provides the processed signal and starting velocity model. The following section (4.1) discusses the important assertions and justifications of normal moveout velocity analysis when applied to study the shallow firn. The concluding section (4.2) discusses the Core 15 Western Spur data analysis and a few causes for circularity inherent to the MxHL methodology.

\subsection{Known Assertions, Errors, and Remediation of NMO Velocity Analysis}

For the reflected wavefront to behave with normal moveout the assertions about the flat-lying and homogeneous snow and firn velocity structure must be upheld. Otherwise, non-hyperbolic travel-time events trend the reflection moveout away from normal. Subsurface events such as dipping beds, velocity heterogeneity, and acquisition geometries that violate the short-spread NMO approximation skew the canonical hyperbolic moveout. Dip moveout and pre-stack depth migration velocity analysis with reflection tomography are common tools to assess complex velocity structure (Deregowski, 1986; Stork, 1992). Bradford et al. (2009a) apply pre-stack depth migration velocity analysis for a multi-channel GPR study on Bench Glacier, Alaska. Their findings show the utility of the method that works around the NMO assumptions when the velocity field expresses complexity due to englacial water content and a lack of planar reflectors.

Barrett et al. (2007) discuss common sources of error within velocity determination experiments conducted via radar CMP analysis under the assumptions of normal moveout. Barrett et al. (2007) address four primary sources of error for the NMO velocity estimate: (1) errors of uncorrected travel-time statics, (2) errors in the mea- 
surement of transmitter and receiver offset, (3) violation of Dix's small-spread approximation, and (4) violation of the flat-lying planar bedded interface assumption. I provide my justfication for the usefulness of the NMO approach by remediating its assertions with the following statements.

I have rigorously corrected for systemic static errors (Appendix B.7, Chapter 2.4.2, and Chapter 2.4.3). Also, I account for outlying travel-time data as a result of systemic or random instrument malfunction. To resolve any unaccounted static errors I apply the $\mathbf{L}_{\mathbf{1}}$ minimization for the rms velocity regression (Chapter 2.4.7). This minimization scheme is applied internally to the residual static correction.

Although the antennas were mounted within the sleds after careful measurement of their separation distances, roughness of the ice sheet surface topography, among other factors of traversing, introduce unavoidable error in the assumed offsets. My argument for resolution of this error relies on the central limit theorem and the understood quasi-normal distribution of random errors existing in Nature (Aster et al., 2013). The signal processing routine aims to attenuate random noise artifacts and bring through the coherent signal. With my best attempt at removing randomly distributed noise from the radar signal, I postulate that positioning errors of the antennas are sufficiently remediated. After random noise removal, out of plane and back scattered signal is sufficiently suppressed. Dip within the planar firn beds is less than $1^{\circ}$. This consideration is modeled and discussed in Appendix A.2.

Within the horizon velocity analysis appendix (D), I formulate an approach that compares Dix's NMO equation with the shifted hyperbola NMO equation. By simulation I show that the array geometry abides the short-spread NMO equation for all wavefield events within the shallow firn. Barrett et al. (2007) conduct an analysis of 
the offset-depth ratio versus errors of the estimated velocity. The authors report for their two layer ice model, with a bedrock interface at $100 \mathrm{~m}$ depth, velocity errors do not exceed $1 \%$ at an offset to depth ratio of 5 . For an annual snow accumulation horizon at $0.5 \mathrm{~m}$ depth, the offset/depth ratio of the $500 \mathrm{MHz}$ multi-channel array is $\sim 24$, almost five fold greater than reported by Barrett et al. (2007). This is the most important assertion to justify, as the surface-coupled wave and the primary reflected wave drive the MxHL modeling and analysis. At the zeroth depth level, analysis of the surface-wave is required. At far offsets the shallow reflection hyperbola asymptotically approaches the surface wave (Burger et al., 2006, ch. 8). A primary reflection at depths not much greater than $0.5 \mathrm{~m}$ is sensible by NMO velocity analysis. I conclude that NMO analysis is viable because the vertical velocity heterogeneity is small. Recall, that the NMO equation (2.6) is exact for a homogeneous medium (Chapter 2.4.5). As vertical velocity heterogeneity increases, biases in the stacking velocity approximation of the rms velocity tend to increase - especially in noisy environments and at later times (Appendix D.3.2). The NMO equation must be expanded to a higher-order approximation (equation D.9) to compensate. Fortunately, the velocity structure of the shallow snow and dry-firn does not require the use of a higher-order

normal moveout equation. This indicates that the stacking velocity, rms velocity, and average velocity are suitably equivalent measures. The results of the MxRadar velocity analysis for depth, density, accumulation, and age modeling are discussed in the following section (4.2).

\subsection{The MxRadar Modeled Approach}

The multi-channel radar analysis (MxHL model) provides a result which quantitatively agrees with the density logs extracted from Core 15 and Pit $15 \mathrm{~W}$ (Figures 
3.4 and 3.5). The primary discussion of this section is the evaluation of the radarinformed-model results between the validation sites. The discussion is weakened by qualitative analysis, but I consider further methods beyond the scope of this thesis which may bring a quantitative assessment of MxHL accuracy. The data analysis was explored to extract the possibilities of the MxHL model (depth, stratigraphy, density, and age). I correlate surface elevation and slope angle as explanation of variability observable in the results. Yet, this analysis is rudimentary and stronger analysis of surface morphology and weather may serve as predictive indicators for the observed density and accumulation patterns.

Qualitatively, I examined the coupling of the mechanical and electromagnetic properties of the firn of Figure 3.9b. The depth of critical density is estimated by the MxHL method and is apparent by the closure of the color contours at the second piecewise intersection of the model $\sim 12 \mathrm{~m}$ depth. An IRH, that displays strong amplitude in Figure 3.7, resides upon this change in the predicted densification regime of the MxHL model. Refer to Figure 3.4; at the critical depth a chicane in the density $\log$ is observed. The change in dielectric permitivity returns a strong amplitude signal from this depth.

I draw quantitative conclusions by comparison of the surface topography (Figure 3.1) with the density anomaly section (Figure 3.9). The relationship of density, elevation, and accumulation is well recognized (Braithwaite et al., 1994; van der Veen et al., 1999; Hamilton, 2004). My study explores the spatial continuity of the relationship between these variables of mass balance. Areas of higher elevation have a negative density deviation while areas of lower elevation have a positive density deviation from the mean. This trend persists from the snow surface through the depth 
image, but is weakened within the névé densification regime. The correlated behavior of snow and firn density variability as a function of elevation is a positive check on the validity of the multi-channel radar methods. Though, elevation is not the sole control of snow and firn densification and accumulation. The continuous spatial structure of snow surface density and mechanical compaction of firn, as measured by Grima et al. (2014b), shows a strong correlation with surface slope angle. The wind-borne redistribution of snow also affects accumulation as a function of slope angle and wind intensity (Winstral \& Marks, 2002; King et al., 2004). Increased surface densification is apparent on the slopes of the topographic trough between $40-45 \mathrm{~km}$ in Figure 3.9b. Improved understanding of the spatial controls on the behavior of snow and firn density can potentially inform air-borne radar accumulation studies (Medley et al., 2013; Karlsson et al., 2016; Lewis et al., 2017) and space-borne radar and lidar surface mass balance studies (Zwally \& Li, 2002; Li \& Zwally, 2011, 2015) as to relax static, ice core density-velocity conversions.

The qualitative agreement among modeled density prediction and the depth converted radiostratigraphy is another practical check on the EM velocity model and the HVA methods. It is important to understand that firn electrical properties, not essentially "annual layers" return radar echos in the shallow firn. And the particular radar bandwidth and pulse type define the resolution and peakedness or lobedness of the returned signal. Age-depth relationship from firn core chemistry (Osterberg et al., 2006) is shown to be a viable method for the age dating of radiostratigraphy, and therefore determining annual snow accumulation. Alternatively, an age-depth relationship was approximated by the MxHL model - based on the accumulation rate estimated for 2016-2017 and assuming this rate is constant over the period 2000-2017 
(Chapter 3.4.3). In regions where melt-refreeze has not significantly altered the predictable density at depth, the MxHL model can derive age-depth scales. The mean annual and interannual accumulation has not yet been estimated for Core 15 by laboratory analysis to evaluate the MxHL age-depth model. 2016 GreenTrACS cores show no significant trend in the mean annual snow accumulation of Southwest Greenland. Interannual variability of accumulation is similar among the reported cores with standard deviation in the range of $0.09-0.120 \mathrm{~m}$ w.e. Shallow firn densification and ice layer fraction from meltwater percolation and refreeze within the firn has significantly increased during the past $\sim 50$ years (Graeter, 2017).

I have not provided a means to quantitatively assess the accuracy of the modeled isochronolgy, as the chemical age-depth relationship is not yet completed; an image cross-correlation between the isochronogram and the radiostratigraphy may provide a quantitative assessment. Though slowly, the firn creeps and the static assessment of the isochronogram needs correction. Additionally, the velocity model provided for depth conversion relies on the same model inputs as the firn chronology. Disentangling the circularity of the method requires the addition of alternative approaches. To rely less heavily on the Herron \& Langway (1980) prediction, horizon velocity analysis of a few additional layers can benefit this problem if the resulting velocity model is satisfactory. Otherwise, more advanced geophysical imaging can be applied. Prestack depth migration can refine the velocity model when provided the MxHL result as a starting iterator (Bradford, 2006; Bradford et al., 2009a,b).

Once stable isotope chemistry of the extracted Core 15 is completed, the modeled and measured isochronology can be compared. Tracing the IRHs of Figure 3.7 from the dated firn core (the conventional method) more accurately reconstructs Figure 
3.10. The reconstructed age-stratigraphy compensates for lateral firn advection, spatially non-static annual precipitation amount, and changes (weather and topographic) to the depositional preference of snow accumulation. The radiostratigraphy driven approach forced by the additional measure of chemical age is more robust, yet more cumbersome. Error within the MxHL mean annual accumulation rate is expected to be small here, because of the good agreement between modeled and measured density (Figure 3.4). Circling back, it is this radar driven agreement which supports the use of the MxHL approach to estimate snow and firn depth, density, accumulation, and age.

An important assumption drives the MxHL modeling procedure; the 2016-2017 annual accumulation estimate is representative of the accumulation history over the previous two decades. In turn, the 2000-2017 prediction is identical to the 20162017 estimate. As discussed above, the accuracy of this assumption has not been tested but is supported by the agreement at Core 15. Geophysical imaging and geochemical dating practices to corroborate or contradict the MxHL methodology have been established. This statement insinuates that future work will be directed to confirm these findings and to explore this method elsewhere on the GrIS. 


\section{CHAPTER 5:}

\section{CONCLUSIONS}

I have developed an approach that estimates the snow and firn density, depth, and accumulation using the GreenTrACS multi-channel radar. The success of this approach depends on the data quality and the ability of the geophysicist to correctly identify wavefield characteristics. Data quality is largely controlled by weather and the GrIS surface conditions. Typically, a deeper snow pack separates the primary reflection return from the surface wave for all offsets, as demonstrated in this thesis. The reflection arrival will interfere with the surface wave for shallow snow and longer offsets. The accumulation of $\sim 30 \mathrm{~cm}$ of fresh snow provides a smooth surface that enhances data quality, but also introduces a bias in the surface density estimate toward the lower values of the fresh snow. Rough surface conditions in the presence

of large sastrugi decreases the coherency of firn radio echos at depth. The signal processing strategy mitigates this problem. Data should be carefully analyzed and interpreted.

The bootstrapped estimate of electromagnetic velocity provides the experimental confidence bounds for the larger ensemble of solutions. The uncertainty analysis flows through the snow and firn quantitative analysis. I have chosen the mean value to represent the radiostratigraphy, density, and age at depth. Though this method proves viable for the study within the dry-snow accumulation zone of the GrIS, it is left 
to be tested within the heart of the western percolation zone and for longer traverses connecting multiple firn cores. This method captures spatial variability of surface snow density and the most recent annual accumulation, yet the steady-state temporal assumptions of the MMxHL model restrict the density and accumulation prediction at depth. A firn compaction model that incorporates horizontal firn advection will improve the prediction of isochrones at depth. Evaluating the MxRadar derived accumulation rate with chemical analysis of Core 15 is necessary to ascertain biases introduced by the steady-state temporal assumption.

I have demonstrated the utility of this ground-penetrating radar and established a strategy for conducting the data analysis. This radar technique elucidates spatial variability in snow accumulation and density which has a predictable influence on the accumulation and density at depth within the dry-snow zone. I have shown that the spatial variability of the surface mass balance components correlates with elevation and surface slope angle and that significant fluctuations in accumulation occur beneath the grid resolution of many polar regional climate model simulations. Indicators for the prediction of accumulation anomalies exist. Perhaps the assimilation of the latest remotely sensed mass balance measures and weather models can be coupled more finely to better understand surface mass balance processes. 


\section{REFERENCES}

Abdalati, Waleed, \& Thomas, Robert H. 2003. Program for Arctic Regional Climate Assessment (PARCA). Page 56 of: van der Veen, C.J. (ed), Greenl. Sci. Plan. Meet.

Al-Chalabi, M. 1974. An Analysis of Stacking, RMS, Average, and Interval Velocities Over a Horizontally Layered Ground. Geophys. Prospect., 22(sep), 458-475.

Alfieri, Lorenzo, Bisselink, Berny, Dottori, Francesco, Naumann, Gustavo, de Roo, Ad, Salamon, Peter, Wyser, Klaus, \& Feyen, Luc. 2017. Global projections of river flood risk in a warmer world. Earth's Futur., jan, 1-12.

Anderson, Don L, \& Benson, Carl S. 1963. The Densification and Diagensis of Snow. Chap. 30, pages 391-411 of: Kingery, W.D. (ed), Ice Snow Prop. Process. Appl. Cambridge, MA: M.I.T. Press.

Arcone, Steven A., Spikes, Vandy B., Hamilton, Gordon S., \& Mayewski, Paul A. 2004. Stratigraphic continuity in $400 \mathrm{MHz}$ short-pulse radar profiles of firn in West Antarctica. Ann. Glaciol., 39(2002), 195-200.

Aster, Richard C., Borchers, Brian, \& Thurber, Clifford H. 2013. Parameter Estimation and Inverse Problems. 2nd edn. Elsevier.

Bader, Henri. 1954. Sorge's law of densification of snow on high polar glaciers. J. Glaciol., 2(15), 319-323.

Barrett, Brian Edward, Murray, Tavi, \& Clark, Roger. 2007. Errors in Radar CMP Velocity Estimates Due to Survey Geometry, and Their Implication for Ice Water Content Estimation. J. Environ. Eng. Geophys., 12(1), 101-111.

Benson, Carl S. 1960. Stratigraphic studies in the snow and firn of the Greenland ice sheet. Dissertation (Ph.D.), California Institute of Technology.

Bindoff, N L, Willebrand, J, Artale, V, Cazenave, A, Gregory, J M, Gulev, S, Hanawa, K, Le Quere, Corinne, Levitus, S, Nojiri, Y, Shum, C K, Talley, L D, \& Unnikrishnan, a S. 2007. Observations: oceanic climate change and sea level. Pages 385-432 of: Clim. Chang. 2007 Phys. Sci. Basis, vol. AR4. 
Box, Jason E. 2013. Greenland ice sheet mass balance reconstruction. Part II: Surface mass balance (1840-2010). J. Clim., 26(18), 6974-6989.

Bradford, J H, Nichols, J, D., Mikesell T, \& Harper, J T. 2009a. Continuous Profiles Of Electromagnetic Wave Velocity and Water Content in Glaciers. Ann. Glaciol., 50(51), 1-9.

Bradford, John H. 2006. Applying reflection tomography in the postmigration domain to multifold ground-penetrating radar data. Geophysics, 71(1), K1.

Bradford, John H., \& Harper, Joel T. 2005. Wave field migration as a tool for estimating spatially continuous radar velocity and water content in glaciers. Geophys. Res. Lett., 32(8), 1-4.

Bradford, John H., Harper, Joel T., \& Brown, Joel. 2009b. Complex dielectric permittivity measurements from ground-penetrating radar data to estimate snow liquid water content in the pendular regime. Water Resour. Res., 45(8), 1-12.

Braithwaite, R. J., Laternser, M., \& Pfeffer, W. T. 1994. Variations of near-surface firn density in the lower accumulation area of the Greenland ice sheet, Pakitsoq, West Greenland. J. Glaciol., 40(136), 477-485.

Brown, Joel, Harper, Joel, Pfeffer, W. Tad, Humphrey, Neil, \& Bradford, John. 2011. High-resolution study of layering within the percolation and soaked facies of the Greenland ice sheet. Ann. Glaciol., 52(59), 35-42.

Brown, Joel, Bradford, John, Harper, Joel, Pfeffer, W. Tad, Humphrey, Neil, \& Mosley-Thompson, Ellen. 2012. Georadar-derived estimates of firn density in the percolation zone, western Greenland ice sheet. J. Geophys. Res. Earth Surf., $117(1), 1-15$.

Brown, Joel, Harper, Joel, \& Humphrey, Neil. 2017. Liquid water content in ice estimated through a full-depth ground radar profile and borehole measurements in western Greenland. Cryosph., 11(1), 669-679.

Burger, H. Robert, Sheehan, Anne F., \& Jones, Craig H. 2006. Introduction to Applied Geophysics: Exploring the Shallow Subsurface. New York, NY: Norton \& Company.

Castle, Richard J. 1994. A theory of normal moveout. Geophysics, 59(6), 983-999.

Chen, Linling, Johannessen, Ola M, Wang, Huijun, Ohmura, Atsumu, \& Chen, Correspondence L L. 2009. Accumulation over the Greenland Ice Sheet as represented in reanalysis data and related atmospheric circulation. 4(1), 11208. 
Daniels, David J. 2009. Antennas. Chap. 4, pages 99-139 of: Gr. Penetrating Radar Theory Appl. Elsevier.

Deregowski, S.M. 1986. What is DMO? First Break, 4(1185), A-1-A-18.

Dix, C. H. 1955. Seismic velocities from surface measurements. Geophysics, 20(1), 68-86.

Efron, B., \& Stein, C. 1981. The Jackknife Estimate of Variance. Ann. Stat., 9(3), $586-596$.

Efron, B., \& Tibshirani, R. 1986. Bootstrap Methods for Standard Errors,Confidence Intervals, and Other Measures of Statistical Accuracy. Stat. Sci., 1(1), 54-77.

Eisen, O., Nixdorf, U., Wilhelms, F., \& Miller, H. 2002. Electromagnetic wave speed in polar ice: Validation of the common-midpoint technique with high-resolution dielectric-profiling and density measurements. Ann. Glaciol., 34, 150-156.

Eisen, Olaf, Nixdorf, Uwe, Wilhelms, Frank, \& Miller, Heinrich. 2004. Age estimates of isochronous reflection horizons by combining ice core, survey, and synthetic radar data. J. Geophys. Res. B Solid Earth, 109(4), 1-11.

Everett, M E. 2013. Near-Surface Applied Geophysics. Cambridge University Press.

Forster, Richard R., Box, Jason E., van den Broeke, Michiel R., Miège, Clément, Burgess, Evan W., van Angelen, Jan H., Lenaerts, Jan T. M., Koenig, Lora S., Paden, John, Lewis, Cameron, Gogineni, S. Prasad, Leuschen, Carl, \& McConnell, Joseph R. 2014. Extensive liquid meltwater storage in firn within the Greenland ice sheet. Nat. Geosci., 7(2), 1-4.

Fowles, G R. 1975. Introduction to Modern Optics. 2nd edn. Holt, Rinehart, and Winston.

Gamble, J.L., Ebi, K. L., Grambsch, A.E., Sussman, F.G., \& Wilbanks, T.J. 2008. Analyses of the Effects of Global Change on Human Health and Welfare and Human Systems.

Gerlitz, Kevin, Knoll, Michael D., Cross, Guy M., Luzitano, Robert D., \& Knight, Rosemary. 1993. Processing Ground Penetrating Radar Data to Improve Resolution of NearSurface Targets. Pages 561-574 of: Symp. Appl. Geophys. to Eng. Environ. Probl. 1993. Environment and Engineering Geophysical Society.

Graeter, Karina A. 2017. Evolution of the W. Greenland percolation zone over the past 50 years. M.Phil. thesis, Dartmouth College. 
Green, C. H. 1938. Velocity Determinations by Means of Reflection Profiles. Geophysics, 3(4), 295-305.

Grima, Cyril, Kofman, Wlodek, Herique, Alain, Orosei, Roberto, \& Seu, Roberto. 2012. Quantitative analysis of Mars surface radar reflectivity at $20 \mathrm{MHz}$. Icarus, 220(1), 84-99.

Grima, Cyril, Schroeder, Dustin M., Blankenship, Donald D., \& Young, Duncan A. 2014a. Planetary landing-zone reconnaissance using ice-penetrating radar data: Concept validation in Antarctica. Planet. Space Sci., 103, 191-204.

Grima, Cyril, Blankenship, Donald D., Young, Duncan A., \& Schroeder, Dustin M. 2014b. Surface slope control on firn density at Thwaites Glacier, West Antarctica: Results from airborne radar sounding. Geophys. Res. Lett., 41(19), 6787-6794.

Gulunay, Necati. 1986. FXDECON and complex wiener prediction filter. Pages 279-281 of: SEG Tech. Progr. Expand. Abstr. 1986. Society of Exploration Geophysicists.

Hamilton, Gordon S. 2004. Topographic control of regional accumulation rate variability at South Pole and implications for ice-core interpretation. Ann. Glaciol., 39, 214-218.

Hawley, R. L., Morris, E. M., Cullen, R., Nixdorf, U., Shepherd, A. P., \& Wingham, D. J. 2006. ASIRAS airborne radar resolves internal annual layers in the dry-snow zone of Greenland. Eur. Sp. Agency, (Special Publ. ESA SP, 33(614), 1-5.

Hawley, Robert L., Courville, Zoe R., Kehrl, Laura M., Lutz, Eric R., Osterberg, Erich C., Overly, Thomas B., \& Wong, Gifford J. 2014. Recent accumulation variability in northwest Greenland from ground-penetrating radar and shallow cores along the Greenland Inland Traverse. J. Glaciol., 60(220), 375-382.

Herron, Michael M, \& Langway, Chester C. 1980. Firn Densification: An Emperical Model. J. Glaciol., 25(93), 373-385.

Jacobel, Robert W., \& Hodge, Steven M. 1995. Radar internal layers from the Greenland Summit. Geophys. Res. Lett., 22(5), 587-590.

Jol, Harry M. 2008. Ground Penetrating Radar Theory and Applications. Elsevier.

Jones, Ian F., Bloor, Robert I., Biondi, Biondo L., \& Etgen, John T. (eds). 2008. Prestack Depth Migration and Velocity Model Building. Society of Exploration Geophysicists. 
Karlsson, Nanna B., Eisen, Olaf, Dahl-Jensen, Dorthe, Freitag, Johannes, Kipfstuhl, Sepp, Lewis, Cameron, Nielsen, Lisbeth T., Paden, John D., Winter, Anna, \& Wilhelms, Frank. 2016. Accumulation Rates during 13112011 CE in North-Central Greenland Derived from Air-Borne Radar Data. Front. Earth Sci., 4(November), $1-18$.

King, J. C., Anderson, P. S., Vaughan, D. G., Mann, G. W., Mobbs, S. D., \& Vosper, S. B. 2004. Wind-borne redistribution of snow across an Antarctic ice rise. $J$. Geophys. Res. D Atmos., 109(11), 1-12.

Legrand, Michel, \& Mayewski, Paul. 1997. Glaciochemistry of polar ice cores: A review. Rev. Geophys., 35(3), 219-243.

Lehmann, F, Horstmeyer, H, Green, A, Sexton, J L, \& Coulibaly, M. 1996. Georadar Data from the Northern Sahara Desert: Problems and Processing Strategies. GPR'96 Proc.

Lewis, Cameron. 2010. Airborne UHF Radar for Fine Resolution Mapping of NearSurface Accumulation Layers in Greenland and Airborne UHF Radar for Fine Resolution Mapping of Near-Surface Accumulation Layers in Greenland and West Antarctica. Thesis (M.S.), University of Kansas.

Lewis, Gabriel, Osterberg, Erich, Hawley, Robert, Whitmore, Brian, \& Marshall, Hans Peter. 2017. Regional Greenland Accumulation Variability from Operation IceBridge Airborne Accumulation Radar. Cryosph., 11(nov), 773-788.

Li, Jun, \& Zwally, H. Jay. 2011. Modeling of firn compaction for estimating ice-sheet mass change from observed ice-sheet elevation change. Ann. Glaciol., 52(59), 1-7.

Li, Jun, \& Zwally, H. Jay. 2015. Response times of ice-sheet surface heights to changes in the rate of Antarctic firn compaction caused by accumulation and temperature variations. J. Glaciol., 61(230), 1037-1047.

Lipscomb, William H., Fyke, Jeremy G., Vizcaíno, Miren, Sacks, William J., Wolfe, Jon, Vertenstein, Mariana, Craig, Anthony, Kluzek, Erik, \& Lawrence, David M. 2013. Implementation and initial evaluation of the glimmer community ice sheet model in the community earth system model. J. Clim., 26(19), 7352-7371.

Looyenga, H. 1965. Dielectric constants of heterogeneous mixtures. Physica, 31(3), 401-406. 
Macgregor, Joseph A, Fahnestock, Mark A, Catania, Ginny A, Paden, John D, Gogineni, S Prasad, Young, S Keith, Rybarski, Susan C, Mabrey, Alexandria N, Wagman, Benjamin M, \& Morlighem, Mathieu. 2015. Radiostratigraphy and age sturcture of the Greenland Ice Sheet. J. Geophys. Res. Earth Surf., 120, 212-241.

Maurer, J. 2006. Local-scale snow accumulation variability on the Greenland ice sheet from ground-penetrating radar (GPR). M.Phil. thesis, University of Colorado, Boulder.

McConnell, Jr, Arthern, R. J., Mosley-Thompson, E, Davis, C, Bales, R, Thomas, R, Burkhart, J, \& Kyne, J. 2000. Changes in Greenland ice sheet elevation attributed primarily to snow accumulation variability. Nature, 406(6798), 877-9.

Medley, B., Joughin, I., Das, S. B., Steig, E. J., Conway, H., Gogineni, S., Criscitiello, A. S., McConnell, J. R., Smith, B. E., Van Den Broeke, M. R., Lenaerts, J. T M, Bromwich, D. H., \& Nicolas, J. P. 2013. Airborne-radar and ice-core observations of annual snow accumulation over Thwaites Glacier, West Antarctica confirm the spatiotemporal variability of global and regional atmospheric models. Geophys. Res. Lett., 40(14).

Moore, Alvin W., \& Jorgenson, James W. 1993. Median filtering for removal of low-frequency background drift. Anal. Chem., 65(2), 188-191.

Moser, T. J. 1991. Shortest path calculation of seismic rays. Geophysics, 56(1), 59-67.

Neidell, N. S., \& Taner, M. Turhan. 1971. Semblance and Other Coherency Measrues for Multichannel Data. Geophysics, 36(3), 482-497.

Ng, F, \& King, E C. 2011. Kinematic waves in polar-firn stratigraphy. 57(206), 1119-1134.

Nghiem, S. V., Hall, D. K., Mote, T. L., Tedesco, M., Albert, M. R., Keegan, K., Shuman, C. A., DiGirolamo, N. E., \& Neumann, G. 2012. The extreme melt across the Greenland ice sheet in 2012. Geophys. Res. Lett., 39(20), 6-11.

Oleson, Keith W., Lawrence, David M, Gordon, B, Flanner, Mark G, Kluzek, Erik, Peter, J, Levis, Samuel, Swenson, Sean C, Thornton, E, \& Feddema, Johannes. 2013. Technical description of version 4.5 of the Community Land Model (CLM). NCAR/TN503+STR NCAR Tech. Note, 422. 
Osterberg, Erich C., Handley, Michael J., Sneed, Sharon B., Mayewski, Paul A., \& Kreutz, Karl J. 2006. Continuous ice core melter system with discrete sampling for major ion, trace element, and stable isotope analyses. Environ. Sci. Technol., 40(10), 3355-3361.

Panzer, Ben, Gomez-Garcia, Daniel, Leuschen, Carl, Paden, John, RodriguezMorales, Fernando, Patel, Aqsa, Markus, Thorsten, Holt, Benjamin, \& Gogineni, Prasad. 2013. An ultra-wideband, microwave radar for measuring snow thickness on sea ice and mapping near-surface internal layers in polar firn. J. Glaciol., 59(214), $244-254$.

Plewes, L A, \& Hubbard, B. 2001. A review of the use of radio-echo sounding in glaciology. Prog. Phys. Geogr., 25(2), 203-236.

Poley, H G. 2010. GPR Antennas Design and Experimental Evaluation. Ph.D. thesis, Delft University of Technology.

Rogriguez, Adolfo, \& Abreu, Raul. 1990. A Mixing Law to Model the Dielectrics Properties of Porous Media. Soc. Pet. Eng.

Sacchi, Mauricio D. 1998. A bootstrap procedure for high-resolution velocity analysis. Geophysics, 63(5), 1716-1725.

Sacchi, Mauricio D. 2008. Seismic Lab. Signal Analysis and Imaging Group: Department of Physics, University of Alberta.

Sasgen, Ingo, van den Broeke, Michiel, Bamber, Jonathan L., Rignot, Eric, Sørensen, Louise Sandberg, Wouters, Bert, Martinec, Zdeněk, Velicogna, Isabella, \& Simonsen, Sebastian B. 2012. Timing and origin of recent regional ice-mass loss in Greenland.

Selesnick, Ivan W., \& Burrus, C. Sidney. 1998. Generalized digital butterworth filter design. IEEE Trans. Signal Process., 46(6), 1688-1694.

Siegert, Martin J, Hodgkins, Richard, \& Dowdeswell, J A. 1998. A chronology for the Dome $\mathrm{C}$ deep ice-core site through radio-echo layer correlation with the Vostok ice core, Antarctica. Geophys. Res. Lett., 25(7), 1019-1022.

Simonsen, Sebastian B., Stenseng, Lars, Adalgeirsdóttir, Gudfinna, Fausto, Robert S., Hvidberg, Christine S., \& Lucas-Picher, Philippe. 2013. Assessing a multilayered dynamic firn-compaction model for Greenland with ASIRAS radar measurements. J. Glaciol., 59(215), 545-558. 
Sørensen, L. S., Simonsen, S. B., Nielsen, K., Lucas-Picher, P., Spada, G., Adalgeirsdottir, G., Forsberg, R., \& Hvidberg, C. S. 2011. Mass balance of the Greenland ice sheet (2003-2008) from ICESat data - The impact of interpolation, sampling and firn density. Cryosphere, 5(1), 173-186.

Steenson, Bernard O. 1951. Radar Methods for the Exploration of Glaciers. Thesis (Dissertation (Ph.D.)), California Institute of Technology.

Stork, Christof. 1992. Reflection tomography in the postmigrated domain. Geophysics, 57(5), 680-692.

Takasu, Tomoji, \& Yasuda, Akio. 2009. Development of the low-cost RTK-GPS receiver with an open source program package RTKLIB. Int. Symp. GPS/GNSS, $4-6$.

Taner, M. Turhan, \& Koehler, Fulton. 1969. Velocity Sprectra-Digital Computer Derivation Applications of Velocity Functions. Geophysics, 34(6), 859-881.

Taurisano, Andrea, Tronstad, Stein, Brandt, Ola, \& Kohler, Jack. 2006. On the use of ground penetrating radar for detecting and reducing crevasse-hazard in Dronning Maud Land, Antarctica. Cold Reg. Sci. Technol., 45(3), 166-177.

Treitel, Sven. 1974. The Complex Wiener Filter. Geophysics, 39(2), 169-173.

Trimble (R. 2008. Trimble @ NetR8 GNSS Reference Receiver User Guide. Tech. rept.

Ulaby, Fawwaz T, Fung, Adrian K, \& Moore, Richard K. 1986. Microwave remote sensing: active and passive. From Theory to Applications, vol. 3. Artech House.

Van den Broeke, M, Bamber, J L, Ettema, J, Rignot, E J, Schrama, E, Van de Berg, W, Van Meijgaard, E, Velicogna, I, \& Wouters, B. 2009. Partitioning recent Greenland mass loss. Science (80-. )., 326(2009), 984-986.

Van Den Broeke, Michiel R., Enderlin, Ellyn M., Howat, Ian M., Kuipers Munneke, Peter, Noël, Brice P.Y., Jan Van De Berg, Willem, Van Meijgaard, Erik, \& Wouters, Bert. 2016. On the recent contribution of the Greenland ice sheet to sea level change. Cryosphere, 10(5), 1933-1946.

van der Veen, C. J., Mosley-Thompson, E., Gow, Anthony J., \& Mark, B. G. 1999. Accumulation at South Pole: Comparison of two 900-year records. J. Geophys. Res., 104(D24), 31067-31076. 
van Kampenhout, Leonardus, Lenaerts, Jan T. M., Lipscomb, William H., Sacks, William J., Lawrence, David M., Slater, Andrew G., \& van den Broeke, Michiel R. 2017. Improving the Representation of Polar Snow and Firn in the Community Earth System Model. J. Adv. Model. Earth Syst., 9(7), 2583-2600.

Waite, A., \& Schmidt, S. 1962. Gross Errors in Height Indication from Pulsed Radar Altimeters Operating over Thick Ice or Snow. Proc. IRE, 50(6), 1515-1520.

Winstral, Adam, \& Marks, Danny. 2002. Simulating wind fields and snow redistribution using terrain-based parameters to model snow accumulation and melt over a semi-arid mountain catchment. Hydrol. Process., 16(18), 3585-3603.

Yilmaz, Öz. 2001. Seismic Data Analysis. Society of Exploration Geophysicists.

Zwally, H. Jay, \& Li, Jun. 2002. Seasonal and interannual variations of firn densification and ice-sheet surface elevation at the Greenland summit. J. Glaciol., 48(161), 199-207. 
APPENDIX A:

\section{DATA ACQUISITION}




\section{A.1 Pulsed Radio Echo Acquisition}

The acquisition mode is a programmable decision within the data logging unit. Two acquisition methods were used during the campaign: odometer and free-run. While running 'odometer' acquisition mode the impulse wavelet is triggered by a wheel coupled to the snow surface. The radar sequences every $\sim 0.25 m(\Delta x)$ while towed and does not trigger while stationary. During 'free run' trace acquisition the radar operates as rapidly as possible. The sequencing frequency is $\sim 20 \mathrm{~Hz}$; approximately 2 cycles of 9 channels are recorded per second. The relative position information is disregarded because the radar collects traces while stationary (Appendix B.3.1). The traversing snow machine operating at $\sim 20 \mathrm{~km} / \mathrm{h}$ acquires traces every $\sim 0.25 \mathrm{~m}$. Navigation at speeds much higher reduces reflection quality by increasing the spatial trace interval and introducing additional antenna bounce. During 'odometer' acquisition operation speeds too fast cause skips in the trace recording, as the instrument is unable to sample the wavefield before triggering another shot (Appendix B.3).

\section{A.2 Frequency Aliasing, Spatial Aliasing, and Stacking}

The sample intervals in time and space contribute significantly to the trade-off between data quality, accuracy, and the rate of acquisition. Applying a longer temporal sample interval reduces the number of samples which compose a fixed recording window, thus, less computation is required to sample a trace and the process occurs more rapidly. Digitized data at frequencies above the Nyquist $\left(f_{N}\right)$ will be aliased, and the proper temporal sample interval $(\Delta t)$ which reconciles the signal frequency 
$\left(f_{s}\right)$ must be selected according to equation (A.1).

$$
f_{s}<f_{N}=\frac{1}{2 \Delta t}
$$

Frequencies above $f_{N}$ are still present as a superposed contamination within the digitized the signal. This affect is know as frequency folding (Yilmaz, 2001, chap. 1). A high-cut, anti-aliasing filter is applied to the analogue signal before digitization to prevent contamination by the unwanted higher frequencies (Yilmaz, 2001, chap. 1).

Spatial aliasing occurs when the wavefront separation in time equals half the dominant period of the $500 \mathrm{MHz}$ wavelet (Yilmaz, 2001, chap. 1). The maximum separable frequency $f_{\max }$ which can be properly imaged may be estimated by equation (A.2) for subsurface velocity $V$, minimum receiver separation $\Delta X$, and dip angle $\theta$.

$$
f_{\max }=\frac{V}{4 \Delta X \sin \theta}
$$

I have created a simple model to determine the effect of spatial aliasing for the array geometry $(\Delta X=1.33 \mathrm{~m})$, average velocity at 25 meters depth $V=0.2 \mathrm{~m} / \mathrm{ns}$, and dip angle $\theta=1^{\circ}$. The calculated $f_{\max }$ from equation (A.2) for these parameters is $2.1541 \mathrm{GHz}$. Topographic features correlate with travel-time dip of reflection horizons, and have a half period of $\sim 2500 \mathrm{~m}$ on GrIS - reported by Hawley et al. (2014) and observed within the GreenTrACS records (Chapter 3). Applying these geographic constraints, the calculated dip angle is $0.5627^{\circ}$. The simple approximation is a safe overestimation of the maximum dip for a layer dipping from the surface to $25 m$ depth. This is an exaggeration of the firn stratigraphy. The dipping of layers is much less and minimizes the affect of spatial aliasing. 
Shot stacking during acquisition increases the signal to noise ratio by a factor of $\sqrt{N}$, where $N$ is number of stacked traces (Everett, 2013, chap. 6). Because the radar array is in constant motion, the stacking of traces is quasi-static and incorporates spatial averaging. Given the desired traverse speed of $\sim 20 \mathrm{~km} / \mathrm{h}$, four traces are stacked within each $0.25 m(\Delta x)$ shot bin. Stacking 4 traces is an adequate minimum for signal enhancement of the field record. 
APPENDIX B:

\section{PREPROCESSING}




\section{B.1 Installation of Acquisition Geometry}

The trace index $(\operatorname{trcIx})$ and distance $(x)$ for cycle $i$ and channel $j$ are calculated by equations B.1 and B.2. The sequence spatial sample interval $(\Delta x)$ is $0.25 \mathrm{~m}$. The sequence interval is always approximate and is relevant primarily during 'odometer' acquisition to prevent trace skip. Channel numbers are provided in Table 2.2.

$$
\begin{aligned}
\operatorname{trcIx} & =\max (\text { channel }) \cdot\left(\text { cycle }_{i}-1\right)+\text { channel }_{j} \\
x & =(\operatorname{trcI} x-1) \cdot \Delta x
\end{aligned}
$$

I calculate the channel offsets by equation (eqn:Offset) after I provide the array geometry ( $t x L o c$ and $r x L o c$ ). The frame of reference for the computation of midpoint location (equation B.4) is $T x 1$ and is the origin during cycle 1; all other antennas are in negative space.

$$
\begin{aligned}
t x L o c & =[0,0,0,-1.33,-1.33,-1.33,-2.67,-2.67,-2.67] \\
r x L o c & =[-4,-8,-12,-4,-8,-12,-4,-8,-12] \\
\text { Offset } & =t x L o c-r x L o c \\
\text { Offset } & =[4,8,12,2.67,6.67,10.67,1.33,5.33,9.33]
\end{aligned}
$$

\section{B.2 De-multiplexing and Data Sorting}

The multi-channel radar data are acquired by antenna pairing. This differs from conventional multi-channel acquisition strategies that acquire data in shot gathers. In either case the raw signal matrix contains all traces with header information. The 
raw traces are in the multiplexed format. Prior to signal processing the traces must be taken from the multiplexed format and arranged into a trace domain. This process is called de-multiplexing. Traces may be sorted into many domains as a function of time or depth. The common-offset domain, common-shot domain, and common-midpoint domain are the primary sortings for multi-channel processes and analysis.

After the skipped, static, or unwanted traces have been removed, and the remaining traces are georeferenced, the traces are de-multiplexed and sorted. The traces are placed into bins for each sorting domain. Each bin of traces is known as a gather. For common-offset gathers a bin exists for each antenna pair. Thousands of traces are placed into each of the few bins. Common-shot and common-midpoint gathers have the transposed dimensions of the common-offset gather. Where $n$ Chan is the number of recorded channels and $n \operatorname{Tr} c$ is the number of traces in the multiplexed format, the bin size is $n C h a n$ and the bin count is $n \operatorname{Trc} / n C h a n$. This assumes each shot/midpoint-offset bin has full fold. A gather of full fold contains a unique trace for each offset/antenna pair. Binning shot and CMP gathers to attain full fold is explained in Subsection B.2.2.

\section{B.2.1 Common-Offset Gathers}

In a common-offset gather the traces are displayed for a single offset (Figure B.2d). Two-way reflection travel time is a function of offset, velocity, and depth. When offset is held constant, variation in the reflection travel-time for a horizon is an attribute of subsurface structure. Though dipping events and reflectors are misplaced, the common-offset domain provides a spatial view of the subsurface. Trace display and processing in this domain is the common practice for single channel radar imaging (Jol, 2008). Processing in the common-offset domain leverages time and space to improve 
the signal in a spatially coherent manner (Appendix C). For additional geophysical analysis the common-offset gathers are resorted into the common-shot or commonmidpoint domains.

\section{B.2.2 Shot Gathers and Common-Midpoint Gathers}

The post-processed data must be sorted into the shot-offset or midpoint-offset domain for velocity analysis and subsequent estimation of the snow density and accumulation. During preprocessing the shot or midpoint locations and trace indices are parsed so the data can be gathered for velocity analysis. The shot or midpoint location is a spatial coordinate that is fixed for a bin of traces.

A shot gather is traditionally defined as the gather of traces at multiple offsets produced by a single shot. Typically the source is the lead of the array with receivers trailing behind - known as a wide angle refraction reflection (WARR) survey. These traces are said to occupy a common shot where each trace has a unique reflection midpoint. During the multi-channel acquisition strategy of the Sensors and Software ${ }^{\circledR}$ equipment, a shot gather is the bin of traces recorded by one cycle of the multi-channel sequencing. A shot gather in this sense is composed of 9 shots - one per antenna pair. The width of the shot gather bin becomes less than the WARR bin because the midpoints are moving while being sequenced.

Each antenna pair occupies a moving midpoint. The discrete midpoint location is the half difference of the receiver and transmitter geometry plus the distance from the starting location $(x)$ (equation B.4).

$$
\text { midpointLoc }=\frac{r x L o c-t x L o c}{2}+x
$$


The midpoint is said to be common when shared by all antenna pairs. This becomes an optimization problem to determine the bin containing the gather of nearest neighboring midpoints; no midpoint is exactly shared under the acquisition geometry. The common-midpoint bin is the collection of traces which most nearly occupies the same reflection point in the firn. A shot or CMP gather has full fold when a trace for each offset is uniquely contained within the optimal bin. A shot gather, under this acquisition strategy acquires full fold on the first cycle of the antenna pairing sequence. The CMP gather achieves full fold and the optimal bin width on the third cycle (Table B.1). Figure B.1 depicts the ray paths through a homogeneous medium for each channel of the shot and CMP gathers.

I implemented an acquisition algorithm that optimizes the width of the CMP bin. Refer to Chapter 2.1 for a review of the acquisition algorithm. If the midpoint location matrix is created such that each column represents a channel and each row represents one cycle, the midpoint matrix can be subdivided into $3 \times 3$ blocks associated with each transmitter antenna. Taking the diagonal of each transmitter block of Table B.1 minimizes the midpoint bin width. By algorithmically incrementing the cycle by one and extracting the diagonal of the proceeding blocks, the common-midpoint locations for the entire transect are computed. The midpoint location (Loc) is the average of the bin (equation B.5), and the bin width is the range of the bin (equation B.6). The spatial interval $(\triangle x M P)$ of the shot and CMP gathers is $\sim 2.25 \mathrm{~m}$ and is equivalent to the distance traveled per cycle (equation B.7). $n$ Chan $=9$ is the number of recorded channels per cycle. 


$$
\begin{aligned}
\text { Loc } & =\frac{\sum \operatorname{Bin}}{n C h a n} \\
\text { Width } & =\max (\text { Bin })-\min (\operatorname{Bin}) \\
\Delta x M P & =n \text { Chan } \cdot \Delta x
\end{aligned}
$$

Table B.1: A reproduction of the programmatic binning procedure. The shot bin is each row of the midpoint location matrix. The CMP bin width is minimized by taking the diagonal of each $3 \times 3$ Tx block. The full CMP fold of 9 traces occurs during cycle 3 . The mean of the bin represents the spatial coordinate and the range of the bin equals its width.

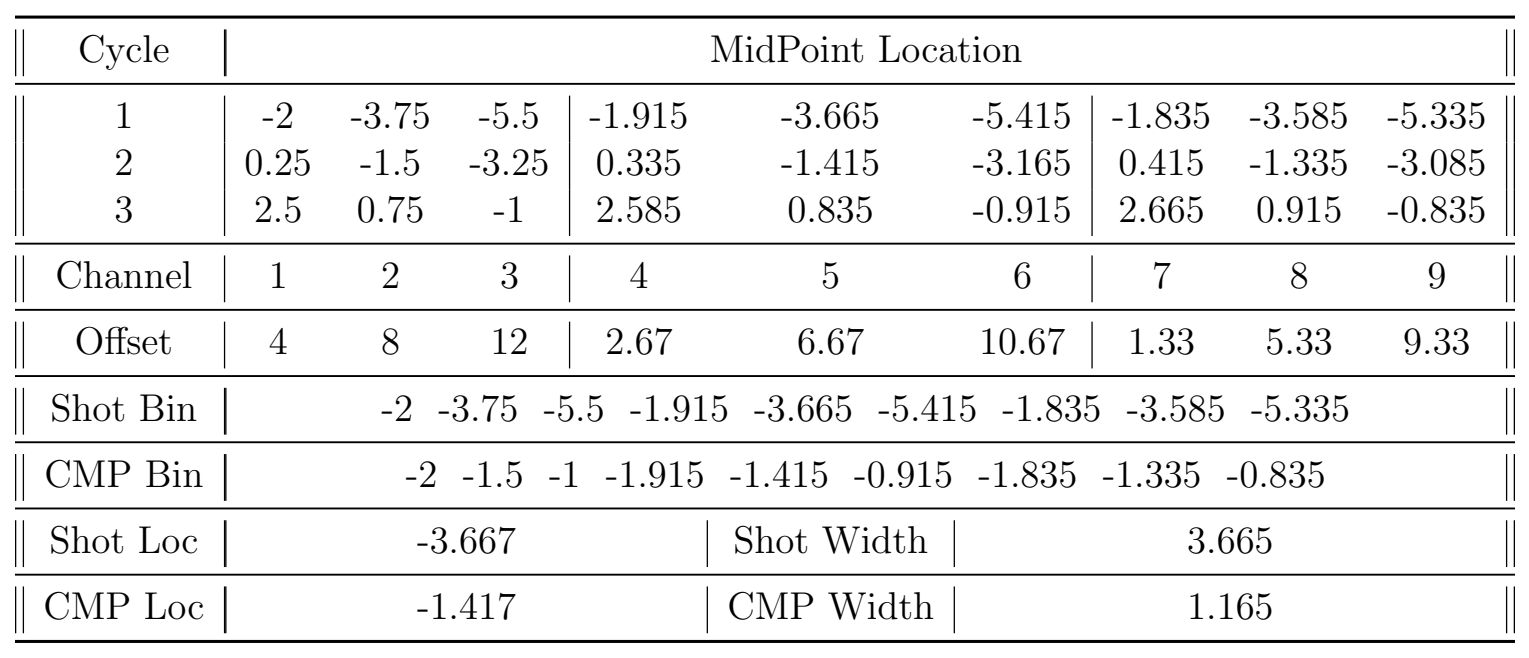



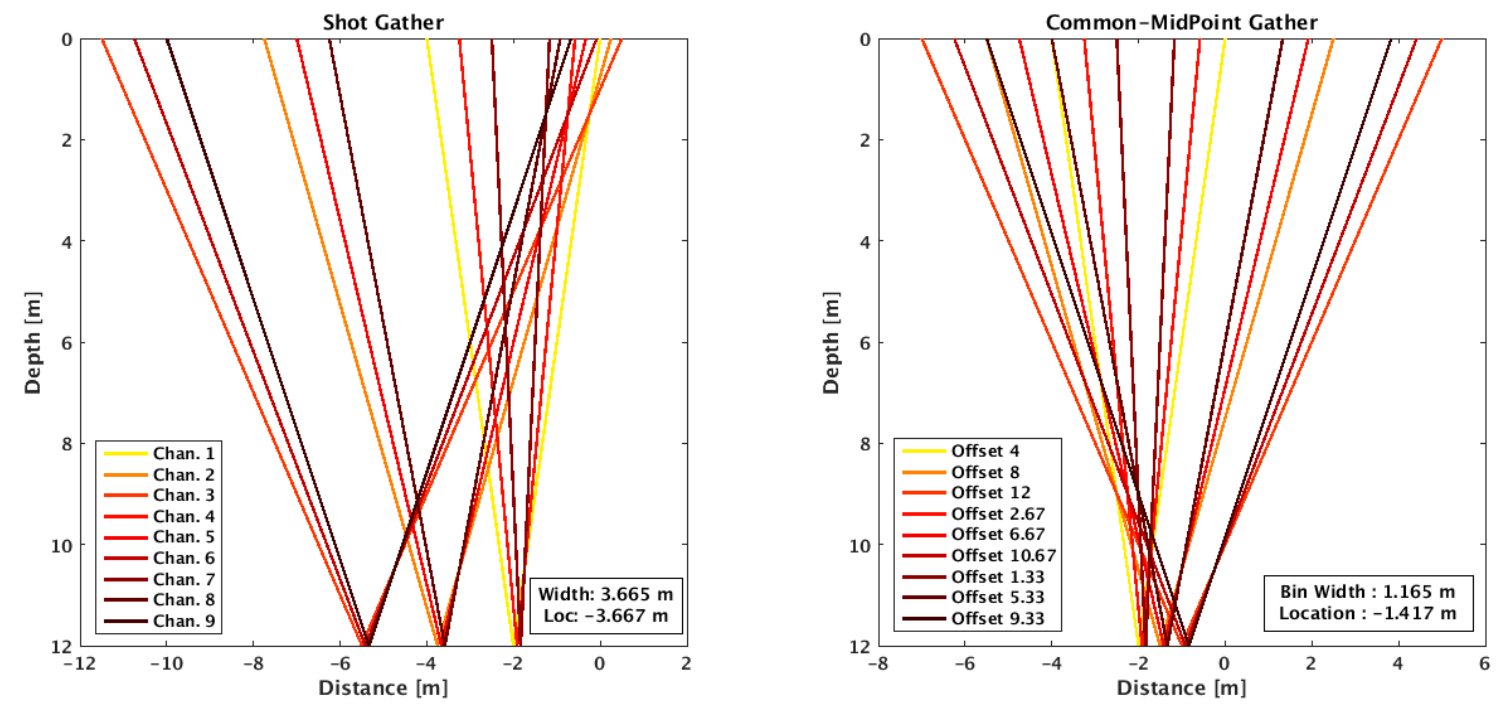

Figure B.1: The ray paths for a shot and CMP gather through a homogeneous velocity medium. This figure emphasizes the different binning strategies for each respective gather. Notice the density and location of reflection points.

\section{B.3 Skipped Trace Removal}

'Odometer' acquisition mode applies a spatial constraint to the recording sequence such that the traces are acquired at an even spatial interval. Skipped traces occur if the GPR system is moving too quickly for signal digitization when triggered by the wheel odometer. A bin of width $\Delta x$ is created for the shot stacking of each trace. If the radar travels a distance greater than $\Delta x$ before a channel is stacked the trace is 'skipped'. The GPR continues to pulse the skipped channel until the stacked trace is acquired. Memory is allocated for each trace, skipped or otherwise. The properly acquired trace is pasted into the multiplex in place of each skipped attempt. This introduces replicated, unwanted traces needing to be removed before sorting into data gathers. The header file contains a flag to indicate skipped traces. The skipped traces are yanked from the multiplex. 


\section{B.3.1 Static Trace Removal}

Static traces accrue while the instrument is idling behind the snowmachine when the radar is operated under 'free-run' trace acquisition. Idle times vary but occur with semi-deterministic character, usually at the beginning and end of a file. Static traces must be removed before georeferencing, mid-point sorting, additional signal processing, and velocity analysis routines.

A static trace is essentially identical to the traces adjacent to itself, because the travel time to a subsurface reflector is constant for any given offset, the energy supplied by the antenna is regulated, and the subsurface reflectivity is unchanging. This principle is fundamental to shot stacking during acquisition, where a stacked trace is the averaged sum of static traces. By examining the covariance of all traces separated by a constant lag distance. I exploit the similarity of the static traces to distinguish them from traces recorded during tow. I will describe my algorithm that removes static traces automatically.

The near-offset channel is tentatively de-multiplexed by calling its traces indexed by the channel number. The near-channel number is determined by a search for the index of the minimum value in the offset array. Then, the common-midpoint gather undergoes the signal processing routine, excluding spatial predictive deconvolution (Appendix C), and is read into the static trace removal function.

The covariance matrix of the near-offset channel is computed in equations B.8 and B.9.

$$
\begin{aligned}
a & =A-\bar{A} \\
\operatorname{Cov}(A) & =\frac{a^{T} a}{N-1}
\end{aligned}
$$


$A$ is the radargram containing static traces, $\bar{A}$ is the mean, $a$ is the deviation score, and $N$ is the number of traces. The diagonal of this matrix contains the variance of each trace and the off-diagonal elements represent the covariance of trace $_{i}$ and trace $_{i+n}$ where $n$ is the lag separation. I expect high covariance for traces that are similar and low covariance for traces that are dissimilar. I have chosen a lag separation of 10 traces, rather, the $10^{t h}$ diagonal of the covariance matrix. This ensures sensitivity to the immediate change in return power once the array begins in tow while looking enough traces ahead to expect a change in the travel time for any reflection arrival. Adjacent traces have increased probability of sharing a higher covariance value. By looking ahead, this approach damps spikes of covariance which may exceed the determined rejection threshold.

I determine a covariance threshold value for rejecting the unwanted traces by examining the cumulative distribution function of the radargram covariance. The covariance CDF is constructed by sorting the covariance values of the $10^{\text {th }}$ diagonal from least to greatest. For a randomly selected trace the CDF expresses the probability of traces which have a covariance less than that of the chosen trace. Provided that the valuable radar signal has low and stable covariance and the unwanted portion of the signal has tremendously high and stable covariance, the covariance CDF resembles a step function with nearly undefined slope at some random probability. I find the probability value where the slope of the CDF dramatically steepens and extract the associated covariance as the rejection threshold.

The slope of the CDF is calculated for each probability as

$$
\frac{\Delta Y}{\Delta X}=\frac{\operatorname{cov}_{i+n}-\operatorname{cov}_{i}}{n},
$$


where $n$ represents the $10^{\text {th }}$ lag separation. Local peaks occur where the derivative of the sign of the slope function is minus one. I locate all peaks along the slope curve and accept only those greater than a threshold (Figure B.2a). The choice of this threshold value is determined by examination of the CDF of the slope. Here, large peaks represent a minuscule portion of the data and exhibit anomalously high slope values. I chose the 0.99 quantile as the probability threshold which defines a peak and extract the index of the first identified peak. This index is mapped to a trace and a covariance which is the covariance threshold for trace rejection. Figure B.2b depicts the covariance associated with each trace. The y-axes on Figures B.2a and B.2b have been normalized for display only. This method produces identical results, supplied the normalized data. The near-channel traces that express covariance within the yellow region are rejected. The program then synthesizes the multiplexed trace indices for the remaining eight channels. These indices are deleted from the multiplexed signal prior to the de-mux routine and geophysical analysis.

Figure B.2 demonstrates an instance where the radar array collected three groups of static traces. The radar sat idle for $\sim 1200$ traces, was then towed past the snow observation pit while recording $\sim 100$ traces, and paused idle again for $\sim 200$ traces while the field team made final preparations. The team then traversed $\sim 15 \mathrm{~km}$ before pausing to save the data. Figures B.2c and B.2d show the near-channel radargram before and after the static trace removal routine. When compared to manual selection of the static traces, the automated routine performs accurately. 


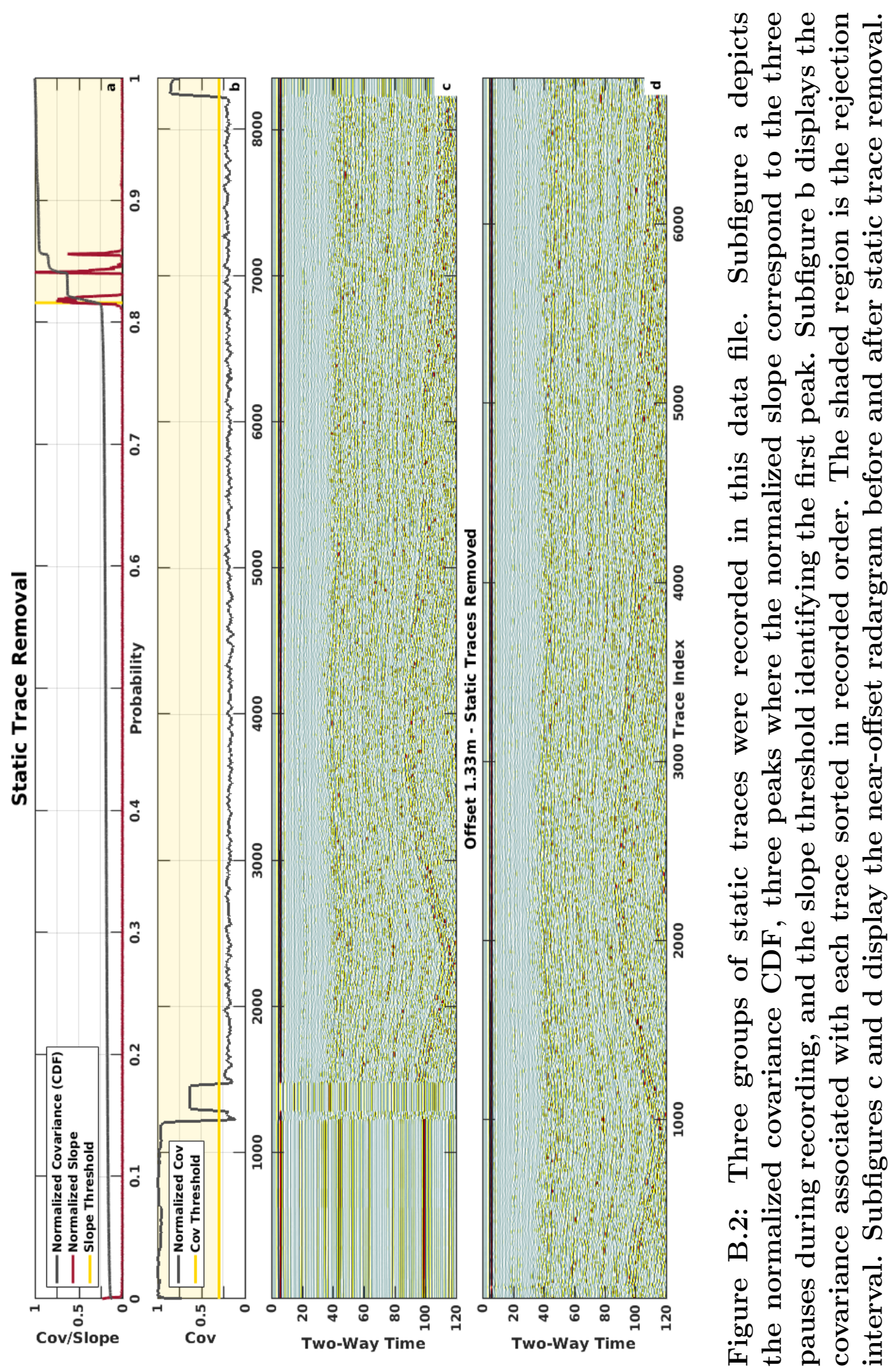




\section{B.4 Trace or Gather Removal}

A gather of traces which display unwanted signal characteristic may be removed from the multiplex prior to signal processing. With prior knowledge, unwanted traces or gathers are removed to ease the memory requirements and CPU overhead while processing the radar signal. Appendix C.1.3 gives justification for removing the nearoffset channel due to the desensitizing effect of inductive coupling. Traces of midpoint gathers may also be removed at random to leverage estimation of parameter uncertainty through a Monte-Carlo sampling technique. The bootstrapping technique is applied for this purpose during the velocity analysis procedure (Chapter 2.4.9).

\section{B.5 Georeferencing Traces}

Global positioning system (GPS) coordinates were acquired contemporaneously with the multi-channel radar. A global navigation satellite system (GNSS) antenna was mounted to the snowmachine and connected to a Trimble ${ }^{\circledR}$ NetR8 receiver (Trimble $($, 2008). The receiver logs the GPS string of coordinates at $1 \mathrm{~Hz}$. An additional, static GNSS NetR8 system was positioned at each camp for the duration of the occupation. The static receiver was used in conjunction with the roving antenna to correct positioning errors caused by local atmospheric and ionospheric phenomena. These corrections were made using RTKLIB, an open source software (Takasu \& Yasuda, 2009).

\section{B.5.1 Static Coordinate Removal}

The post-processed GPS log is parsed into segments which mate with the files output from the skipped/static trace removal routine. Radar and GPS information are synced by a sudden change in the state of the signal caused by motion of the 
snowmachine. Like the method for identifying the static state of the radar (Subsection B.3.1) there exists an identifiable change in the GPS signal.

\section{B.5.2 GPS Coordinate Interpolation}

The longitude, latitude, and elevation sampling is decimated by a factor of $\sim$ 20, compared to the trace acquisition frequency $\left(f_{\text {trc }}\right)$ of $\sim 20 \mathrm{~Hz}$. The decimated GPS coordinates are evenly distributed into the multiplexed trace header. A linear interpolation is used to infill the untagged trace headers. Because the GPR array is located by a single GPS receiver, a dead-reckoning correction is applied that assumes a static distance from the GPS to each GPR antenna pair midpoint. Otherwise, due to the midpoint sequencing, the GPS information becomes smeared. Each trace in the multiplexed header and data base is now properly georeferenced.

\section{B.6 Effect of Skipped Traces and Tow Speed on Midpoint Binning}

The radar experiences acceleration while being towed because of the surface roughness condition or 'whiskey throttle' of the operator. During 'odometer' acquisition, acceleration to speeds greater than the trace acquisition rate $\left(f_{\operatorname{trc}} \Delta x\right)$ induces the skipping effect (Subsection B.3).

Trace skipping moves a discrete midpoint-gather location and increases the bin width. Let $\mathbf{q}$ be a vector formed by the columns of an array with dimensions $n$ Chan $\mathrm{x} n$ Cycle that indicates the number of skips on a channel per binning cycle. $n C y c l e$ is one for shot gathers and three for common-midpoint gathers. The cumulative summation of $\mathbf{q}$, is computed by the causal integration operator $\mathbf{G} . \Delta L o c$ are position perturbations (equation B.12). This vector accounts for the distance gap accumulated 
by the recorded traces until full fold is met; consider only the indices within the shot or CMP bin. The updated bin location (B.13) and bin width (B.14) are calculated as in Subsection B.2.2, after applying the position perturbations.

$$
\begin{aligned}
\mathbf{G}=\left[\begin{array}{lll}
1 & & 0 \\
\vdots & \ddots & \\
1 & \cdots & 1
\end{array}\right] \\
\Delta L o c=\mathbf{G q} \Delta x \\
L o c^{*}=\frac{\sum(\operatorname{Bin}+\Delta L o c)}{n C h a n} \\
W i d t h^{*}=\max (\operatorname{Bin}+\Delta L o c)-\min (\operatorname{Bin}+\Delta L o c)
\end{aligned}
$$

The spatial trace interval is flexible when operating in 'free run' mode. As the velocity of the towed array changes so does $\Delta x$. This causes stretching or shortening of the midpoint bin - similar to the effect of trace skipping. However, no reflection information is lost due to the acceleration. The radar array is always collecting the optimal bin width for the current trace sample rate and the effect smoothly averages the reflection profile.

For either acquisition strategy every trace position is reckoned after the GPS information is interpolated (Subsection B.5.2). The common-midpoint bins may be geodetically reconfigured provided the reckoned trace positions. But little advantage is gained. Because the stratigraphic variability of the snow and firn happens gradually over distances of kilometers, the residual errors due to traces skipping and tow velocity can be ignored. I proceed through to the velocity analysis method in the shotgather domain (Chapter 2.4), using the shot gathers created during the initial binning procedure (Subsection B.2.2). 


\section{B.7 Systemic Time-Zero Correction}

Waveform sampling errors incorrectly position the recorded trace in time. The bulk affected travel time errors are caused path length variation of information transit circuitry, especially including the lengthened $R x 3$ cable, and random fluctuation within the instrument's reckoning of time-zero when re-booted. Throughout the GreenTrACS campaign the radar equipment was calibrated to estimate the random and systemic malfunctions of the hardware. A coarse scale and necessary correction for EM wave arrival time perturbation is applied during prepossessing. Instrument calibration was performed rigorously in the field during the 2017 campaign and ex-

perimentally under cold laboratory conditions for 2016 to create a global systemic calibration correction for direct air wave arrival time. The air wave is expected to travel at $\sim 0.3 \mathrm{~m} / n s$, and I impose that recordings must agree. This is a cruxical fact for reducing snow velocity estimates from known-source radar travel-times. Chapter 2.4.3 explains the additional remediation of residual error.

By picking the direct air wave arrival across all channels under controlled experimental conditions, I observed the recorded air wave moveout velocity to be much less than theoretical $(c)$. Systemically averaged shifts applied to the individual arrival times for each channel were calculated by forcing the measured air arrival to the theoretical value $(c)$. A sample population of 16 calibrations were assimilated to construct the baseline correction datum. I apply a circular resampling shift of the radar information to the averaged theoretical constraint of the multi-channel arrival times. This effectively repositions the wavefield information to its approximately correct arrival time. To truely recover the reflected amplitudes (Appendix C.2) and image the wavefield accurately, the systemic shifts are applied before signal processing. 


\section{APPENDIX C: SIGNAL PROCESSING}




\section{C.1 De-WOW Filtering}

The goal of De-WOW filtering is to remove the pervasive, resonant, low-frequency signal component and additional instrument DC-bias from each trace. Signal 'WOW' is a low-frequency component that imposes a positive (or negative) trend on the signal amplitude spectrum (Jol, 2008, chap. 5). WOW is the result of near-field coupling effects between the antennas and the ice sheet surface. Additionally 'WOW' is created by the saturation of electrical components when excited by the large amplitude direct arrival waves. The excitation of sensor electronics by the EM field causes resonant inductive coupling ('ringing') within the antenna and circuitry.

A trace after proper De-WOW filtering will have a mean of zero, indicating that the low-frequency amplitude bias has been removed. Gerlitz et al. (1993) present three methods for signal De-WOW: residual median filtering, residual mean filtering, and band-pass filtering. I have found that joining these filters performs the best for subtracting the WOW, DC-bias, and random noise.

\section{C.1.1 Residual Median Filtering}

Residual median filtering has the capability to remove random noise while maintaining signal characteristic (Moore \& Jorgenson, 1993). The residual median filter computes the median of the trace within a gate of signal samples and assigns the median to sample $i$ at the center of the gate. This gate slides along the trace and recomputes for all samples of the trace. The residual, or the noise, is effectively filtered from the radargram by replacing each sample through this process.

The gate of the residual median filter is centered about sample $i$ and has length $2 n_{s}+1$. Shown in equation C.1, $\left(n_{s}\right)$ the number of samples was decided by the nominal frequency $f_{o}=500 \mathrm{MHz}$ and the sample interval $d t=0.2 \mathrm{~ns}$. For these 
parameters $2 n_{s}+1=21$.

$$
n_{s}=\frac{1}{f_{o} d t}
$$

The gate length is tapered on the edges such that the backward or forward looking directions are progressively lengthened or shortened.

I found that lowering the frequency, or lengthening the gate, below the nominal frequency tended to remove structure of dipping reflectors. Though slight, DC-bias was still present. The resulting signal is greatly improved with the addition of residual median filtering. Figure C.1 displays residual of the raw signal median (the noise matrix) for two filter lengths.

\section{C.1.2 Residual Mean Filtering}

After random noise attenuation of the residual median filter, remnant DC-bias must be subtracted. Recall that a properly De-WOW'd trace has a mean of zero with positive and negative amplitudes distributed about zero. Residual mean filtering aims to ensure this. The residual of the mean is otherwise referred to as the deviation score (equation B.8). The deviation score is computed for all samples of each trace. I effectively filter the residual of the mean for each trace by replacing the datum with its deviation score. This promotes the mean of each trace to zero.

\section{C.1.3 Band-Pass Filtering}

In addition to the statistical residual filters I apply a two octave band-pass filter centered about $500 \mathrm{MHz}$. I identify the spectral peaks of low frequency WOW and the reflection signal for the row sum of all raw amplitudes and the De-WOW filtered signal. I have highlighted the WOW component in Figure C.2, following Gerlitz et al. (1993). After reviewing the content of the 9 channels presented in Figure C.2, 

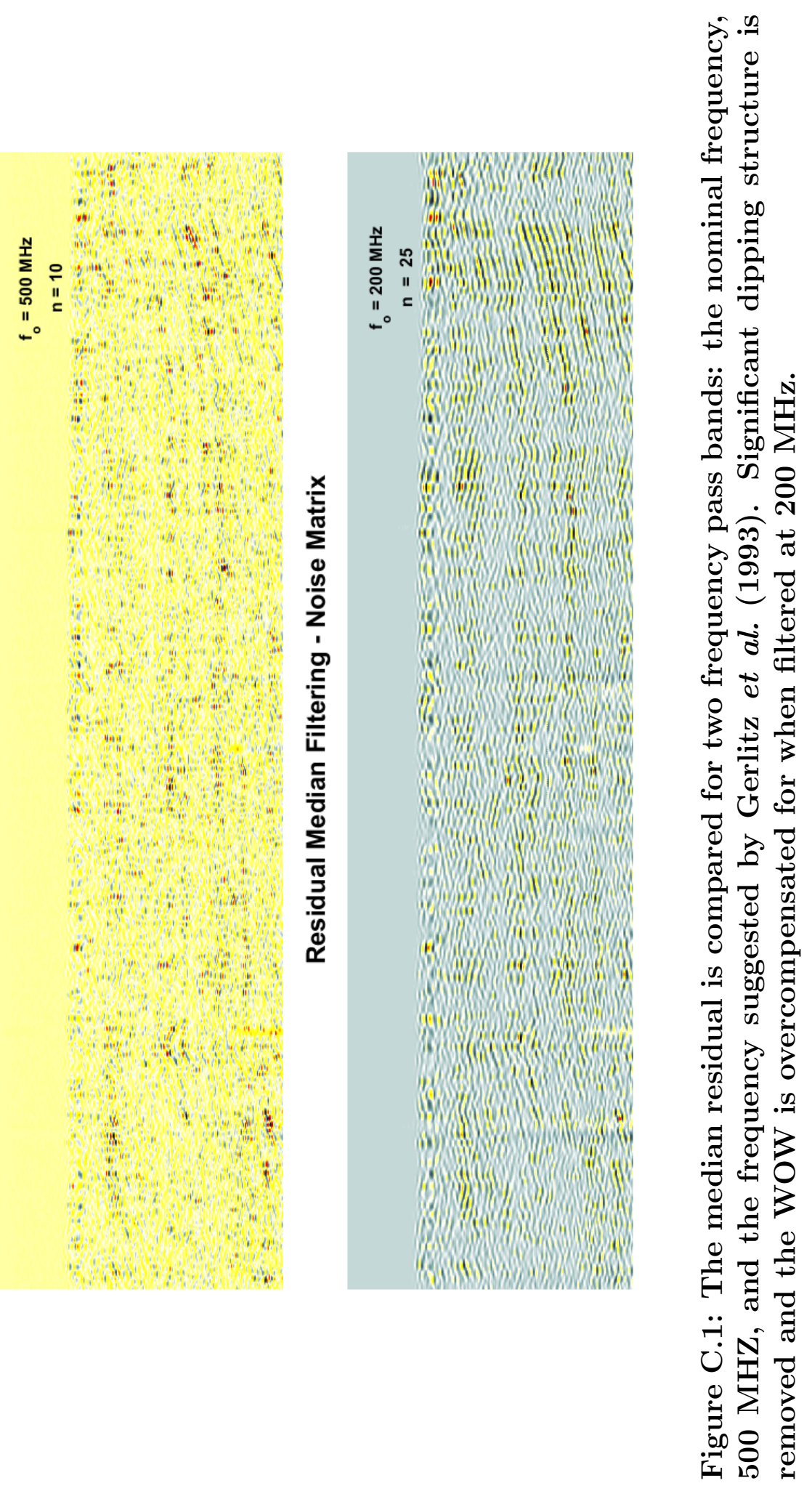
I targeted the frequency $\frac{2}{5} f_{o}$ as the threshold for the frequency-domain, band-pass filter. I selected an $8^{\text {th }}$ order butterworth design (Selesnick \& Burrus, 1998) which employs $-48 d B$ gain per octave beyond the two octave window to curtail spectral leakage into the 'WOW' domain.
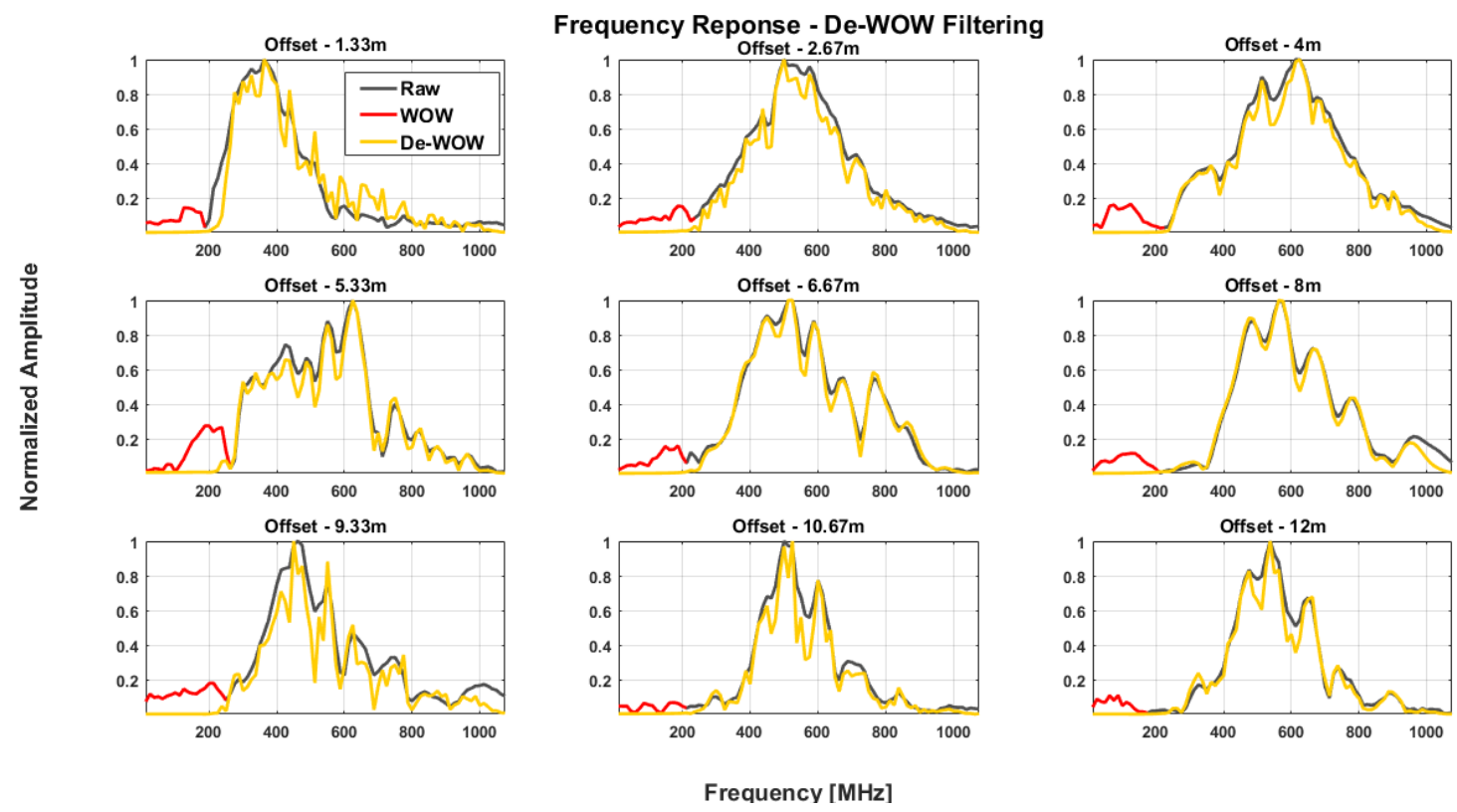

Figure C.2: The trough separating the 'WOW' and the reflection signal was identified by Gerlitz et al. (1993). I have highlighted the WOW component in red. The targeted frequency stop is $\sim 200 \mathrm{MHz}$ or $\frac{2}{5} f_{o}$ and typically represents the cutoff frequency for ' $\mathrm{WOW}$ ' attenuation. The yellow, filtered, signal approaches zero amplitude near the $\frac{2}{5} f_{o}$ threshold.

\section{C.1.4 Kill Channels}

Each channel presents a different spectral fingerprint (Figure C.2). In particular, the near-offset $(1.33 \mathrm{~m})$ channel displays a narrow bandwidth and a bulk frequency down shift centered about $350 \mathrm{MHz}$. This channel also suffers from dreaded 'ringing' (Lehmann et al., 1996); immense resonant inductive coupling persists at the nearoffset as the electronics remain saturated with induced electromagnetic energy, post 
filtering. Reflection horizons are muddled with consistent ringing of the direct arrival amplitudes. The near-offset channel was removed from signal processing and horizon interpretation for the benefit of reduced uncertainty and increased accuracy of traveltime inversion. To visually identify travel-time horizons the signal must be amplified, or gained, during signal processing (Section C.2).

\section{C.2 Amplitude Recovery Gain}

As the radar pulse propagates through the snow and firn, the wavefront diverges spherically. The wavelet energy becomes spread over a sphere of increasing radius $r$, thus diminishing. Within a homogeneous medium the energy density diminishes proportionately to $\frac{1}{r^{2}}$. As energy is the square of amplitude, wave amplitudes diminish

as $\frac{1}{r}$ (Yilmaz, 2001, chap. 1). Snow and firn are not homogeneous media, they display many horizontal discontinuities of electrical impedance and scattering sources. Energy is partitioned at boundaries of electrical impedance and to a low degree absorbed by the firn. These are known as attenuation losses. The objective of amplitude recovery gain is to regain energy losses of wavefront divergence while maintaining the relative amplitudes associated with signal reflection and transmission across horizons. Valuable information of the velocity structure is kept within the reflectivity profile; best practice is to maintain this information.

\section{C.2.1 Recovery of Cable Attenuation}

Analogue signal is transmitted through radio-frequency suppressed, highly conductive, cables to the digitizing unit. The typical antenna cable is $10 \mathrm{~m}$ in length; however, to reach the farthest receiving antenna a cable $17 \mathrm{~m}$ in length was used. The raw signal experiences antennuative losses due to electrical resistance heating during transmission. I apply amplitude correction for the far offset channels by scalar 
multiplication of 1.7 or $\frac{17 m}{10 m}$. This constant scaling sufficiently balances the relative amplitudes of the far offset channels with the unaffected near and mid-offset channels.

\section{C.2.2 Time-variant Exponential Gain}

A well recognized method for recovering the loss due to spherical divergence within a layered-earth is t-squared scaling (Yilmaz, 2001, chap. 1). Where $t$ is a vector of time samples and $\alpha$ is any real number exponent, the exponential gain function (equation C.2)

$$
\begin{aligned}
& g(t)=t^{\alpha} \\
& S(t)=s(t) g(t)
\end{aligned}
$$

scales the signal $(s(t))$ by multiplication of the exponentiated time-series (equation C.3). For t-squared scaling $\alpha$ is 2 . The true amplitudes of the recorded signal are approximately recovered by t-squared scaling. When true amplitude recovery is not the objective of the processing flow, during horizon picking for example, $\alpha$ may be scaled higher to enhance reflector brightness at depth. Alternatively, a method for balancing amplitudes throughout the trace is presented in subsection C.2.3.

\section{C.2.3 Instantaneous AGC Gain}

Automatic gain control (AGC) is another approach of time-variant amplitude scaling that comes at the expense of true amplitude recovery. AGC re-scales the amplitude structure of a trace to enhance the visual quality of reflections large and small. Selection of the filter gate length must be chosen carefully as to not damage the signal character. A time gate too small will amplify the signal and noise greatly such that the relative balance of weak and strong reflections is unclear. A gate too 
large will introduce a shadow zone following larger amplitude reflections, fainting subsequent arrivals (Yilmaz, 2001, chap. 1).

The instantaneous computation of AGC scales each sample of a trace by a ratio of the root-mean-squared amplitude to the square of this value. I used the instantaneous AGC gain function developed by the Seismic Lab group (Sacchi, 2008) and provide their computation in equations C.4 - C.6.

$$
\begin{aligned}
s(t)_{r m s} & =\sqrt{\frac{s(t)^{2} * w}{\sum w}} \\
g(t) & =\frac{s(t)_{r m s}}{s(t)_{r m s}^{2}} \\
S(t) & =s(t) g(t)
\end{aligned}
$$

The gate length is determined by the length of $w$; a triangular weighting kernel. The convolution operation $(*)$ of the squared-signal $\left(s(t)^{2}\right)$ and $w$ effectively computes a weighted mean of the squared amplitudes, when divided by the sum of the weights. The square root of this operation computes the weighted RMS amplitudes. Assigning a weighted kernel is purposeful and robust. Weighting allows the gain function sensitivity to the sample in consideration and its nearest neighbors while damping signal at a distance that may have a different amplitude and phase relationship.

I apply AGC after spatial predictive deconvolution (Subsection C.5) to boost amplitudes of signal used in the semi-automated horizon picking algorithm (Appendix D.1). AGC does well at increasing the sharpness of peaks and troughs in the signal. This is important when interpreting a radargram which displays successive specular reflections. A gate length of 25-50 samples boosts the low amplitude features, while a 350 sample gate balances the amplitudes of the entire time section. 


\section{C.3 Trace Stacking}

Time-variant filtering (Section C.1) reduces the noise along the time dimension of each trace; however, random, uncorrelated noise persists in the spatial dimension (Yilmaz, 2001, chap. 6). In a similar approach to shot stacking during acquisition, averaging adjacent traces improves the signal to noise ratio by filtering incoherent, random artifacts. Stacking increases the smoothness and lateral continuity of the reflection signal. I stack traces by computing the moving weighted mean along the image rows; each row of the signal matrix $(s(t))$ is convolved with a bell-shaped weighting kernel $(w)$ and divided by the sum of the weights (equation C.7).

$$
S(t)=\frac{s(t) * w}{\sum w}
$$

I chose a hamming window of length 21 for $w$. With trace spacing of $\sim 2.5 m$ the stacking function is applied across $\sim 25 \mathrm{~m}$. At this distance the weighted mean trace stacking acts on the spatial frequencies as a high cut filter. Significant variation in the snow and firn structure occurs on length scales $\sim 500 \mathrm{~m}$ or greater. Figure C.3 displays the random noise removed by the trace stacking process. 


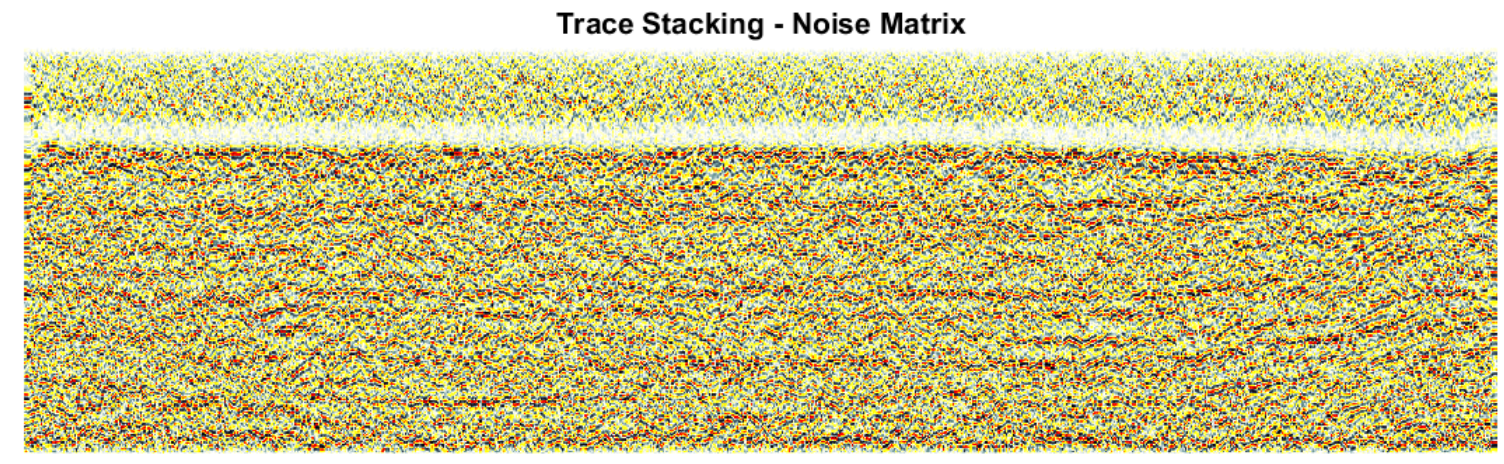

Figure C.3: The noise removed during the stacking process. The noise matrix has AGC applied within a 50 sample window to amplify the noise for visual representation. This image does not depict the true noise level.

\section{C.4 Spatial Median Subtraction}

Coherent noise contaminates the reflection data. Impedance contrasts within the electronics hardware emanate consistent background signal into the reflection data. Changes in the operational temperature of the radar hardware cause variation in the amplitude of the noise. Prior to spatial prediction filtering, I subtract the median trace calculated from the traces within large, overlapping, spatial gates. Flat lying amplitude horizons, such as the direct arrival events and reflections with small travel-time dip, will be removed through this process. Subtracting the direct arrival information will have an adverse effect on the horizon velocity analysis. To remedy this, I apply the spatial median filter only to later arrival times. Large spatial gates containing 1000 traces or more are used to capture the subtle variation of travel-time amplitude information such that reflection arrivals are preserved. Figure C.4 is the coherent instrument noise removed by the spatial subtraction filter. 


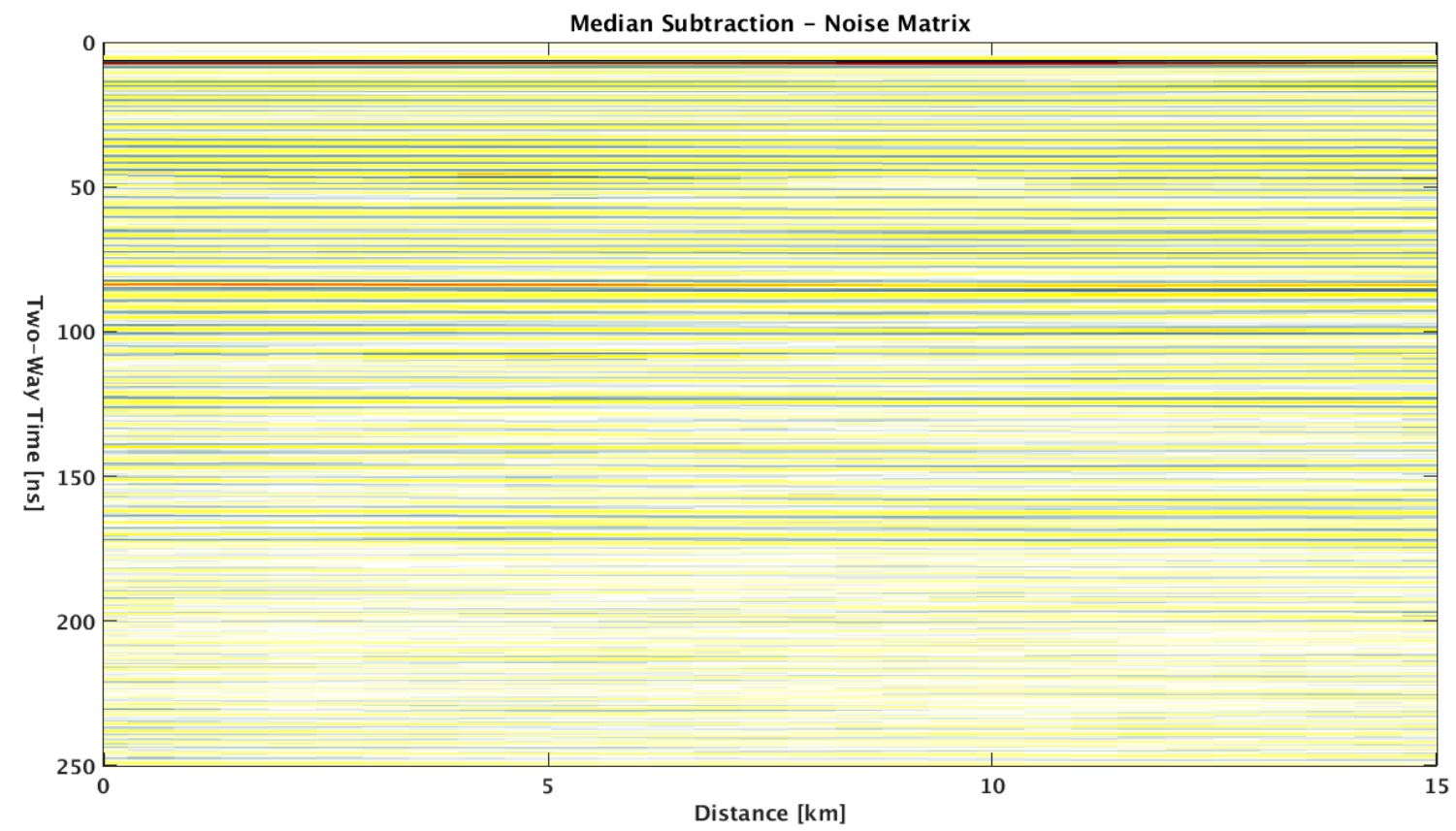

Figure C.4: Coherent instrument noise is removed by subtracting the trace median of overlapping spatial gates. Removal of the linear banding enhances the reflection quality. Dimming in the noise matrix around 50 ns reveals reflection information which is preserved through this process. In this example the median was subtracted from all travel times of the nearest offset $(1.33 \mathrm{~m})$ channel.

\section{C.5 Spatial Predictive Deconvolution}

Spatial prediction filters have the robust property of attenuating random noise events in the presence of complex subsurface structure. Because spatially random noise is uncorrelated among traces of the gathered section and is independent of temporal frequency (see figure C.3), a noise-reduction predictive filter may operate in the frequency-space (f-x) domain (Yilmaz, 2001, chap. 6). Coherent signal is predicted by a convolution of the optimum complex Wiener filter with the Toeplitz matrix formed of the frequency transformed gather. The error of the least-squares prediction is understood as the random noise component. Formulation of the complex Toeplitz 
matrix and the complex Wiener filter is provided within Treitel (1974). Clarity on the implementation of the f-x deconvolution method can be found within Gulunay (1986). Below, I describe the implementation of the least-squares operator to estimate the complex Wiener filter.

A gather of traces is first transformed into the frequency-space domain by computation of the Fast Fourier Transform (FFT). The number of discrete frequencies that comprise the signal estimate depends on the number of time samples of the input gather, the sample rate, and the chosen frequency band of the computation. The chosen frequency band is the same pass-band of the butterworth filter - a two-octave window centered on the nominal frequency (Subsection C.1.3). For each discrete frequency the filter is estimated and the noise is removed.

Sacchi (2008) estimates the complex Wiener filter coefficients using forward and backward autoregression models. The model matrix $(\mathbf{G})$ of the forward approach is the complex Toeplitz matrix. $\mathbf{G}$ of the backwards approach is the complex Hankle matrix. The psuedoinverse is applied to solve the filter coefficients $m$ (Aster et al., 2013). $\mathbf{d}_{\text {pred }}$ is a prediction of the data $\mathbf{d}$ and is estimated when computed forward (equations C.8 and C.9).

$$
\begin{aligned}
\mathbf{m} & =\left(\mathbf{G}^{\mathbf{T}} \mathrm{G}+\mathrm{W}\right)^{-1} \mathbf{G}^{\mathbf{T}} \mathbf{d} \\
\mathbf{d}_{\text {pred }} & =\mathbf{G m}
\end{aligned}
$$

$\mathbf{W}$ is the diagonal weighting matrix formed by the diagonal elements of $\mathbf{G}^{\mathbf{T}} \mathbf{G}$ multiplied by $\mu$ the pre-whitening condition. $\mu$ is a damping term which informs the inversion of the acceptable noise level. 
The forward and backward predictions are averaged for each discrete frequency slice. The complex conjugate of the predictions are calculated to honor the possible symmetries of the complex solution. The data is transformed back to the time domain via the inverse FFT. The common-offset gather passed into the $\mathrm{f}$-x deconvolution is parsed by overlapping time gates. I pass blocks containing $N$ sequential samples of each trace into the operator. The gate advances $\frac{N}{2}$ samples, the operation is recomputed, and the overlapping samples are averaged. This technique relaxes the time-invariant assumptions of predictive deconvolution. Figure C.5 displays the benefit of spatial predictive deconvolution as compared to the input image produced by the aforementioned processing flow. 

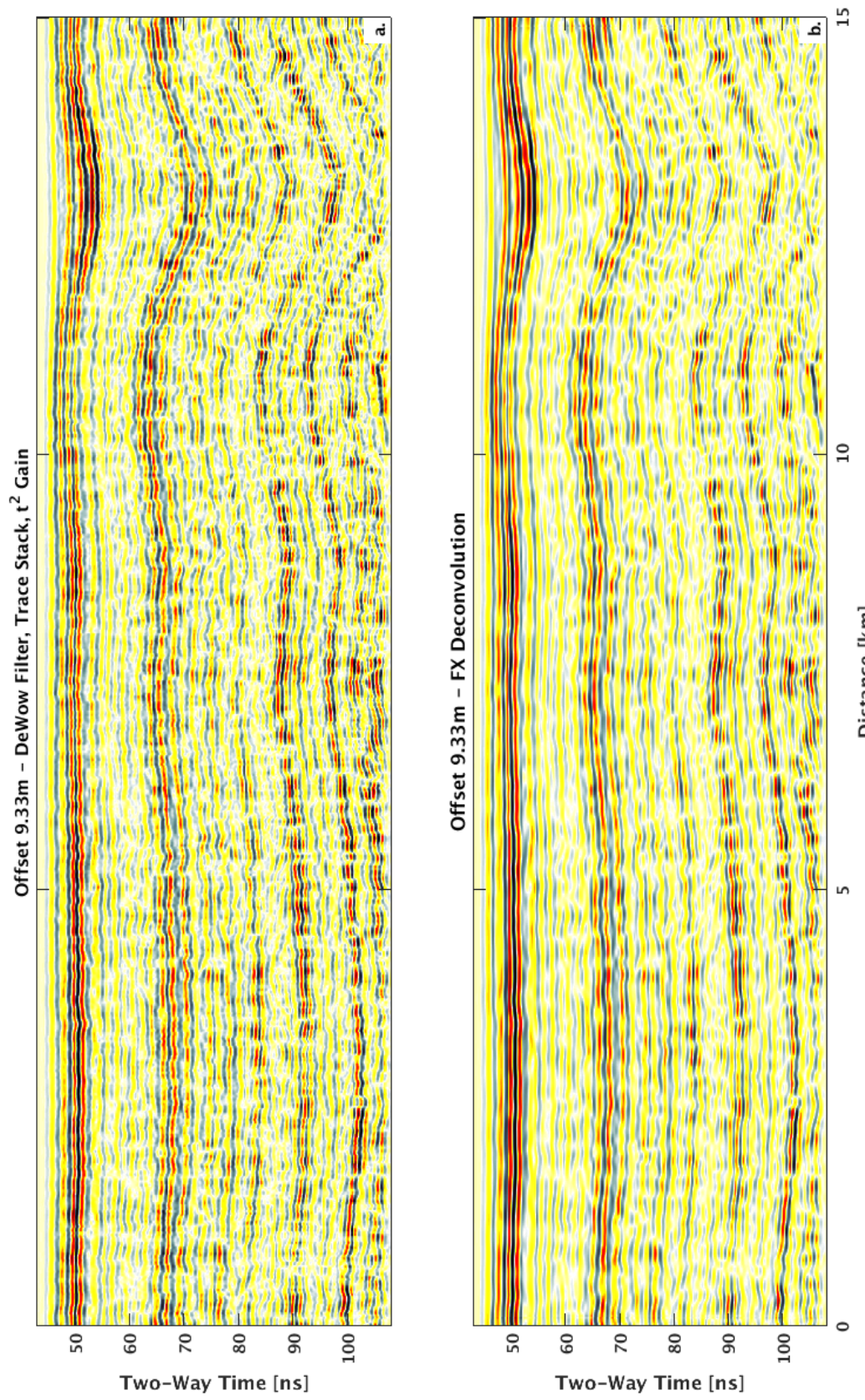

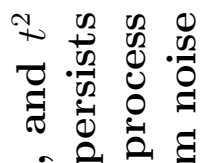

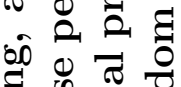

莗

仓

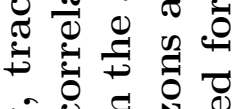

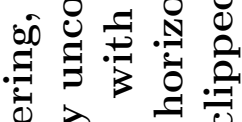

壳

उ

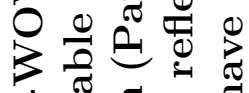

ఏ

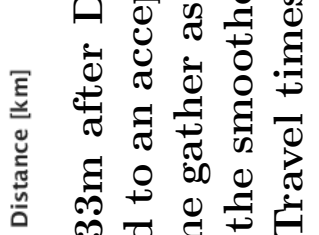

ต

$\dot{0}$ D

*

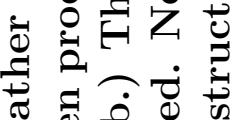

$\infty 00 \%$

包产虽

刍

Е

ชิ

Ð

คే

ن̈

ن

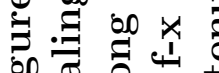

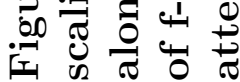




\section{C.6 Normal Moveout Correction and Depth Conversion}

\section{C.6.1 Stacking Velocity}

The signal to this point has been processed for velocity analysis (Chapter 2.4). Following the horizon velocity analysis, a stacking velocity model is compiled. This is a 2-D model in time and space that expresses the wave velocity for wavepaths that abide the assumptions of normal moveout. This model is interpolated such that each time sample of every trace on record is represented by a stacking velocity. Necessary for time-depth conversion, this model may become a matrix of depths following equation (D.18).

\section{C.6.2 Normal Moveout Correction and Stretch}

The normal moveout correction projects the reflected energy recorded on a channel with finite offset to zero-offset (Yilmaz, 2001, chap. 3). The aim of this process is to flatten the reflections recorded on shot gathers so the gathers may be stacked horizontally to produce a single image. The proper stacking velocity will project the traces to zero-offset correctly such that stacking will strengthen coherent reflections and attenuate random noise. Following the normal moveout equation (2.6), the NMO correction is the difference between the arrival time for a given offset $t$ and the theoretical zero-offset time $t_{0}$ calculated from the stacking velocity $\left(V_{r m s}\right)$ (equation C.10).

$$
\Delta t_{N M O}=t-t_{0}
$$


Normal moveout correction is performed on each time sample of a trace. The amplitude of a time sample for a given offset is placed at the calculated zero-offset time via $\Delta t_{N M O}$. A weighted linear interpolation is used if the calculated $t_{0}$ is within the instrument sample rate (Sacchi, 2008). This procedure occurs for each channel for all midpoint locations of the radar transect.

An affect of the NMO correction is signal stretch. Signal of early travel-times at farther offsets becomes stretched on the time axis after the resampling of the NMO correction because $\Delta t_{N M O}$ is significantly large. Yilmaz (2001, chap. 3) reviews the fundementals of stretch and its remediation - stretch muting. I have not applied stretch muting to compensate for the stretch affect. Rather, I reapply the De-WOW filter (Section C.1) to extinguish the undesired low frequencies caused by stretch while preserving the underlying signal characteristic.

\section{C.6.3 CMP Stacking}

The normal moveout corrected and stretch compensated offset gathers closely maintain signal versus travel-time characteristics, dependent on the correctness of the stacking velocity model. CMP stacking is the summation or averaging of the gathered traces to increase the coherent signal strength and attenuate random noise. Like shot stacking in the field, midpoint gather stacking increases the signal to noise ratio by a factor of $\sqrt{N}$, where $N$ is number of stacked traces (Everett, 2013, Chap. $6)$. The stacked section reduces the many offset gathers into a single depiction of the subsurface stratigraphy. Stacking can be done selectively over a group of traces in a gather, known as a partial stack. Figure 3.7 is the partial stack of offsets $4 m$ and $12 \mathrm{~m}$. 


\section{C.6.4 Time to Depth Conversion}

The stacked section is a profile view of the snow and firn in the travel-time domain. During depth conversion, the stacking velocity model is applied to produce an image in depth. Time to depth conversion resamples the stacked section to a depth axis by a 1D bandlimited sinc function interpolation. A matrix of depths is composed from the stacking velocity model (equation D.18). Each sample of the stacked image is represented by a calculated depth. I extract the minimum and maximum depths from the matrix and create a depth axis which increase by $d z$. To avoid depth aliasing I choose the appropriate $d z$ according to equation (C.11) for $V_{\min }$ the minimum velocity of the model and $d t$ the sample interval in the time domain.

$$
d z<\frac{V_{\min } d t}{2}
$$

The post-stack and depth converted image is fed back into the f-x deconvolution routine (Section C.5) to produce the final stratigraphic section. The signal processing routine is concluded with the depth section but is interrelated with the horizon velocity analysis discussed in Section 2.4. The depth converted image can be directly linked to nearby firn cores for the depth-age analysis of snow accumulation rate or overlain on the predicted depth-density section (Chapter 3). 


\section{APPENDIX D:}

\section{HORIZON VELOCITY ANALYSIS}




\section{D.1 Semi-Automated Waveform Travel-time}

\section{Picking}

I developed a semi-automated predictive horizon picking platform to ensure proper selection of wavefield events. polarPicker.m is a seeded picking routine that tracks the peak, trough, or zero amplitude of the selected horizon. The user initially plants the seed with a cross-hair by a graphical user interface and has the capability to reseed the pick if the amplitude matching algorithm goes awry. The program offers the capability to pick $N$ horizons for direct and reflected arrivals. Care must be taken to choose the same phase of each horizon across all channels. The predictive horizon picking algorithm uses a slant-stack approach in polar coordinates. I describe the computer algorithm below.

A forward looking window of the radar image is centered about the seed pick. Keeping the true $(x, t)$ position aside, I assign relative coordinates $\left(x^{*}, t^{*}\right)$ within the window such that the pick is the origin of a two-quadrant grid. The amplitudes within

the window are normalized on a $[-1,1]$ scale. I transform the $\left(x^{*}, t^{*}\right)$ coordinates of the image into polar space such that each discrete amplitude within the window now has polar coordinates $(r, \theta) . r$ is radial distance and $\theta$ is the forward direction $[0, \pi]$, where the 0 angle is looking in the positive time direction. The polar coordinate projection is calculated by equations (D.1) and (D.2).

$$
\begin{aligned}
& r=\sqrt{t^{* 2}+x^{* 2}} \\
& \theta=\sin ^{-1}\left(\frac{x^{*}}{r}\right)
\end{aligned}
$$


For each discrete $\theta$, the average of the amplitudes along the radial path is computed. The predicted pick depends on the desired amplitude attribute (peak, trough, or zero) and the direction $(\theta)$ which optimizes the average of the attribute. The optimum coordinates $\left(r^{\prime}, \theta^{\prime}\right)$ are given a prime notation. The predicted pick $\left(x^{\prime}, t^{\prime}\right)$ has the spatial coordinate $x^{\prime}$ given in equation (D.3) and has the time coordinate $t^{\prime}$ calculated by equation (D.4).

$$
\begin{aligned}
& x^{\prime}=x+n \\
& t^{\prime}=t+r^{\prime} \cos \theta^{\prime}
\end{aligned}
$$

The window advances a predetermined distance $n$ traces with each iteration of the algorithm. While $x^{\prime}$ may be determined as $r^{\prime}$, increasing $x^{\prime}$ incrementally is less susceptible to malfunction and is better for memory management.

\section{D.1.1 Distance Weighted Interpolation of Travel-Time Picks}

Because the pick density is decimated by a factor $n$, the picks are smoothly interpolated by a distance weighting scheme. For each interpolated pick $\left(x_{i}, t_{i}\right)$ of the horizon the distance to its neighbors is calculated (equation D.5). A threshold distance $(R)$ (equation D.6) determines the nearest neighbors $(i x)$ whose picks are gathered for the interpolation. I applied the bi-squared kernel $(w)$ (equation D.7) to calculate the distance weights to be applied to the neighboring picks. The interpolated pick $\left(t_{i}\right)$ of any horizon is the weighted mean of the neighboring picks (equation D.8). 


$$
\begin{aligned}
d & =\sqrt{\left(x-x_{i}\right)^{2}} \\
i x & =d<=R \\
w & =\left(1-\left(\frac{d(i x)}{R}\right)^{2}\right)^{2} \\
t_{i} & =\frac{\sum w * t(i x)}{\sum w}
\end{aligned}
$$

\section{D.2 Higher-Order NMO Approximation}

Castle (1994) provides a derivation of the shifted hyperbola NMO equation (D.9). The shifted NMO equation is an approximation of the higher-order normal moveout equation initially derived by Taner \& Koehler (1969). The shifted NMO equation is exact through the fourth-order of Taner \& Koehler's (1969) expansion and is a very good approximation to the sixth-order term as well. Dix's (1955) approximation is exact through the second order. By expanding the normal moveout equation to a higher degree polynomial, stacking velocity bias related to velocity heterogeneity, which stems non-hyperbolic behavior, is lessened in practice.

$$
t=t_{0}\left(1-\frac{1}{S(x)}\right)+\sqrt{\left(\frac{t_{0}}{S(x)}\right)^{2}+\frac{x^{2}}{S(x) V_{r m s}^{2}}}
$$

$S(x)$ is the fourth order expansion term (equation D.10) which is found by rearrangement of equation (D.9). When $S(x)$ is sufficiently large the higher order 
terms vanish leaving the short-spread NMO equation.

$$
S(x)=\frac{\frac{x^{2}}{V_{r m s}^{2}}-2 t_{0}\left(t-t_{0}\right)}{\left(t-t_{0}\right)^{2}}
$$

\section{D.3 Dix's Equations to Determine Interval}

\section{Velocity}

The interval velocity is determined from the rms velocities estimated at the horizons bounding a layer. The rms velocity for each horizon is estimated by the least squares regression (Chapter 2.4.7; equation 2.7). It is seen by equation (D.11) how the estimated velocity from the $n^{\text {th }}$ horizon is the rms velocity. Dix's (1955) expression of the rms velocity (D.11) is given for the general case of the $n^{\text {th }}$ subsurface horizon, where $\Delta t_{n}$ is the one-way vertical travel time through the $n^{t h}$ layer. The one-way travel-time through the $n^{\text {th }}$ layer is the difference between the vertical travel-time of any two juxtaposed interfaces (equation D.14).

$$
V_{n_{r m s}}=\sqrt{\frac{\sum_{i=1}^{n} V_{i n t_{i}}{ }^{2} \Delta t_{i}}{\sum_{i=1}^{n} \Delta t_{i}}}
$$

Similarly, $V_{r m s}$ of the shallower layer is given by (D.12).

$$
V_{n-1_{r m s}}=\sqrt{\frac{\sum_{i=1}^{n-1} V_{\text {int }_{i}}{ }^{2} \Delta t_{i}}{\sum_{i=1}^{n-1} \Delta t_{i}}}
$$

The zero-offset travel time to any firn horizon is twice the sum of the $\Delta t$ vector (equation D.13). Recall that $t_{0}$ is estimated by least squares regression. 


$$
\begin{aligned}
t_{0 n} & =2 \sum_{i=1}^{n} \Delta t_{i} \\
\Delta t_{n} & =\frac{t_{0 n}-t_{0 n-1}}{2}
\end{aligned}
$$

Dix's method takes advantage of $V_{r m s}$ and $\Delta t_{n}$ between adjacent horizons to compute an interval velocity for any layer. Equation (D.15) is known as the Dix Equation.

$$
V_{i n t_{n}}=\sqrt{\frac{V_{n_{r m s}}^{2} t_{0 n}-V_{n-1_{r m s}}^{2} t_{0 n-1}}{t_{0 n}-t_{0 n-1}}}
$$

The vertical thickness of any layer is equal to the interval velocity times the oneway travel-time through the layer interface (equation D.16). The sum of all layer thicknesses through $h_{n}$ yields the depth $z_{n}$ of the $n^{\text {th }}$ firn horizon (equation D.17).

$$
\begin{aligned}
& h_{n}=V_{\text {int }_{n}} \cdot \Delta t_{n} \\
& z_{n}=\sum_{i=1}^{n} h_{i}
\end{aligned}
$$

The depth of the $n^{\text {th }}$ horizon is equivocal to equation (D.18) if the rms velocity is approximately equal to the average velocity.

$$
z_{n}=\frac{V_{n_{r m s}} \cdot t_{0_{n}}}{2}
$$




\section{D.3.1 Matrix Equation to Determine Interval Velocity}

For a direct solution of the interval velocity model, Dix's equation (D.15) may be cast into a matrix equation. For a 1-D stacking velocity model $V_{r m s}$ that has $n$ layers of uniform thickness, the integration operator $\mathbf{G}$ is the causal integration operator (equation B.11) and $\mathbf{w}-$ a weighting vector - is the row sum of $\mathbf{G}$; $\odot$ is the elementwise Hadamard product. The interval velocities are computed by equations (D.19) and (D.20).

$$
\begin{aligned}
\mathbf{d} & =V_{r m s}^{2} \odot \mathbf{w} \\
V_{\text {int }} & =\sqrt{\mathbf{G}^{-\mathbf{1}} \mathbf{d}}
\end{aligned}
$$

Often, the subsurface model is composed of irregularly sampled horizons. Dix (1955) reconciles this problem by assigning weights in the form of equation (D.14) to the interval velocity calculation. To compose this method as a linear system of equations G becomes the $n \times n$ lower triangular matrix with each column wise element equal to the one-way travel time through the $n^{\text {th }}$ layer. Again, w is a weighting vector equal to the row sum of G. Equations (D.19) and (D.20) are computed to yield the same result as in the recursive solution (D.15).

To arrive at the stacking velocity model from a velocity converted density log of a core site (Section 2.5) or a density-depth curve extrapolated from an empirical model (Subsection 2.5.3) the cumulative average is computed. Posing the problem of equations (D.19) and (D.20) in the forward direction gives the stacking velocity profile. For a 1-D interval velocity model $V_{i n t}$ which has $n$ layers of ambiguous thickness, the integration operator $\mathbf{G}$ reflects the appropriate interval measures; the stacking 
velocities are computed by equation (D.21).

$$
V_{\text {stack }}=\mathbf{w}^{-\mathbf{1}} \odot \mathbf{G} V_{\text {int }}
$$

Calculation of equations (D.19 - D.21) operates in either the time or depth domain. The appropriate weights ( $\Delta t$ or $h$ ) are assigned within $\mathbf{G}$ to change domains.

\section{D.3.2 Testing Dix's Equations}

I conducted an exercise to examine inherent errors in estimating $V_{r m s}$ and $V_{\text {int }}$ via Dix's solution. A depth-density profile extracted from GreenTrACS Core Site 7 is reproduced as EM velocities in Figure D.1. The method of transforming dry snow/firn densities into an estimate of the EM velocity is explained within Chapter (2.5). Provided $V_{\text {int }}$ and the interval thickness from the core measurements, I synthesized zero-offset one-way travel times via equation (D.16) and two-way reflection travel-times for 9 offsets (Table 2.1) via equation (2.6). The velocities estimated from

the core measurements are represented in black. $V_{a v g}$ (Figure D.1a) is estimated by equation (D.22) for each interval $n . V_{r m s}$ is estimated by equation (D.11).

$$
V_{a v g_{n}}=\frac{z_{n}}{\sum_{i=1}^{n} \Delta t_{i}}
$$

A significant caveat of Dix's solution is instability. Dix's equation (D.15) is susceptible to large errors when the difference in one-way travel time through an interval is small relative to the change in velocity. Though the recovery of the Core 7 interval velocity is excellent, noise contributions in the travel-time picks deteriorate the stability of this solution. The resulting interval velocities for synthetic cases with noisy travel-time estimates are presented within subsection (2.4.8). 
Al-Chalabi (1974) provides the heterogeneity factor $(g)$ as a vertical velocity heterogeneity measure. $g$ (equation D.23)is zero for a homogeneous medium and progressively increases as the velocity heterogeneity increases (Figure D.2a). Deviation in $g$ is much less than $1 \%$ for the Core 7 travel-time data.

$$
g=\frac{V a v g^{2}-V_{r m s}^{2}}{V_{a v g}}
$$

Because $g$ is small, the stacking velocity is a good estimate of $V_{r m s}$. This relationship underpins the analysis of stacking velocities to estimate $V_{r m s}$ and $V_{\text {int }}$ via Dix's equation (D.15). Stacking velocity bias $(B)$ (equation D.24) increases with increasing $g$ and ray parameter (Al-Chalabi, 1974). The EM ray parameter tends to increase with depth.

$$
B=V_{\text {stack }}-V_{\text {rms }}
$$

Random noise was added to the synthesized reflection travel-times from Gaussian distributions of $\mu=0$ and $\sigma=[1: 5] d t$. Stacking velocity was estimated by leastsquares fit to the reflection travel-times following the $x^{2}-t^{2}$ method (Chapter 2.4.6). Figure D.2b presents the mean stacking velocity bias of 1000 travel-time simulations for each depth at low and high noise levels. $B$ is below the velocity sensitivity threshold of this analysis, at $\sigma=1 d t$, indicating that the stacking velocity is a good measure of the rms velocity. Because negative errors in travel-time data create larger velocity inaccuracies than positive errors (Al-Chalabi, 1974), at $\sigma=5 d t$ the mean stacking velocity is skewed from the low error $(\sigma=1 d t)$ stacking velocity bias. Errors of this type are increased at higher noise, greater offsets, and later arrival times. For $\sigma=5 d t$, average stacking velocity inaccuracies are $\sim 0.5 \%$ and are $\sim 1.2 \%$ at maximum. 

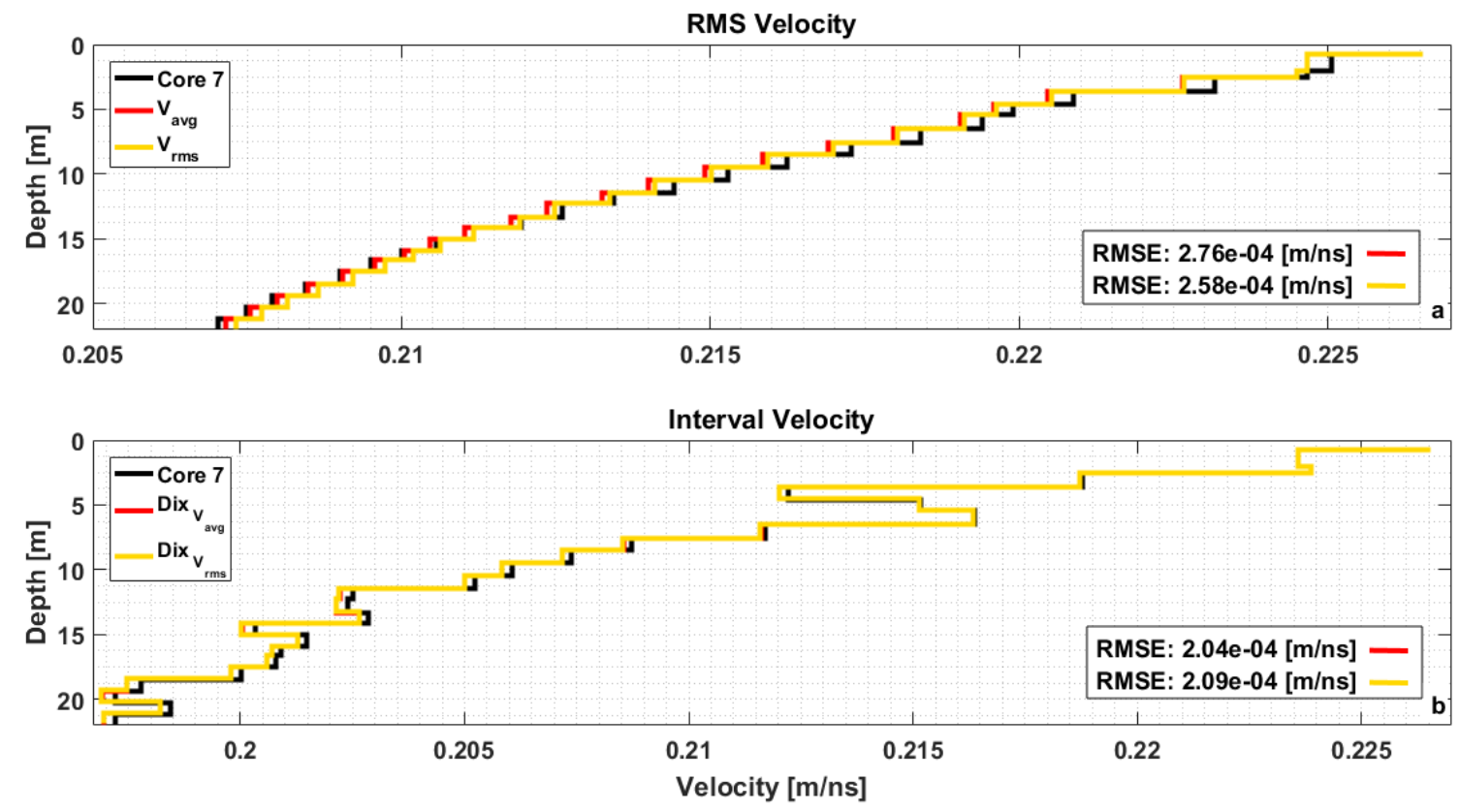

Figure D.1: The GreenTrACS 2016 Core Site 7 depth-density profile is represented as average velocity and rms velocity (Panel a.) by equations (D.22) and (D.11). Interval velocity (Panel b.) is estimated by supplying both $V_{\text {avg }}$ and $V_{\text {rms }}$ into Dix's equation (D.15). The interval densities extracted from Core 7 are transformed into EM velocities via the CRIM equation (Chapter 2.5). The estimation of $V_{\text {rms }}$ agrees well with $V_{\text {avg }}$, and each of these measures agree well with the core data observable by the low root-mean-square error (RMSE) statistic. The velocity axis has been exaggerated to make the small error visible. 

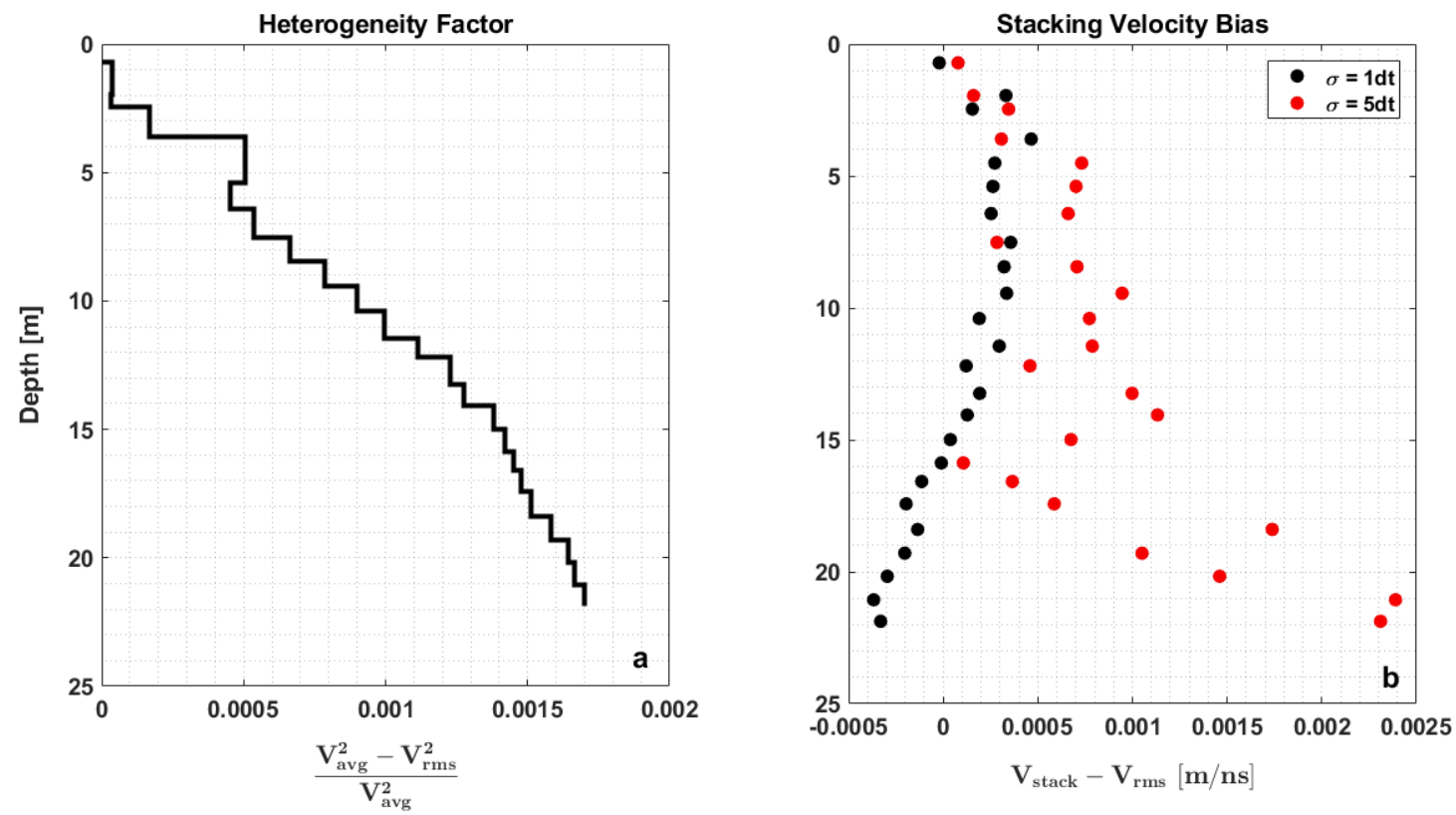

Figure D.2: Al-Chalabi (1974) provides the velocity heterogeneity factor (g) and stacking velocity bias (B) as a tool for understanding the relationship between $\mathrm{V}_{\text {avg }}, \mathrm{V}_{\text {rms }}$, and $\mathrm{V}_{\text {stack }}$. 


\section{D.4 Monte-Carlo Travel-time Simulations to Determine $\mathbf{L}_{1}$ Convergence Factor $\tau$}

I generated synthetic travel-times for both direct wave (LMO) and reflected wave (NMO) cases by supplying the array geometry, the rms velocity model, and the synthesized zero-offset intercept time $\left(t_{0}\right)$ into equations (2.1) and (2.6). I derived both rms and interval EM velocity models by applying the CRIM equation (2.27) to a firn core density-depth profile extracted at Core Site 7 in 2016. The accuracy of the velocity model construction and solution recovery is demonstrated in Section (D.3.2). Travel-time horizons were allowed to be the $10 \mathrm{~cm}$ depth intervals of the snow pit from the fresh surface $(0 \mathrm{~m})$ to the drilling surface $(0.7 \mathrm{~m})$ for the LMO calculations. For NMO calculations travel-time horizons are the time converted run interval of extracted core segments. The thickness of the extracted Core 7 runs range from $0.515 m$ to $1.24 m$ with $\sigma=0.154 m$.

I created 5 Gaussian noise distributions with zero mean $(\mu=0)$ and standard deviations $(\sigma)$ ranging from $1 d t$ to $5 d t$. I added randomly sampled noise from the distributions to the synthetic travel-times. For each noise level I synthesized 10 shot records to recreate the spatial averaging of this many shot gathers during the bootstrapping approach to velocity analysis (Chapter 2.4.9). In this simulation the LMO and NMO velocities were estimated by the IRLS approach. I allowed the inversion scheme to iterate beyond a reasonable convergence factor. The LMO and rms velocity of the 10 synthetic travel-time records are averaged to produce an experimental $z-V_{L M O}$ and $z-V_{r m s}$ profile. I then supplied the $z-V_{r m s}$ information into Dix's

equation (D.15) to estimate the interval velocities (Figures D.3b, D.4b, and D.5b). 
Because Dix's equation (D.15) is susceptible to large errors when the difference in one-way travel time through an interval is small relative to the change in velocity (the thin layer case), I decimated the rms velocity profile by a factor of 2 prior to Dix's solution. I applied the distance weighted interpolation scheme (Section D.1.1) to the nearest 3 neighbors of the estimated rms velocity model, where the interpolant decimates the number of samples. By decreasing the resolution of this experiment not only does the Dix (1955) solution stabilize, but also the firn intervals are represented in a manner that is more true of observable horizons picked on the real data.

$$
R M S E=\sqrt{\frac{1}{N} \sum_{i=1}^{N}\left(V_{\text {core }_{i}}-V_{\text {est }_{i}}\right)^{2}}
$$

I calculated the root-mean-square error (equation D.25) of the snow pit model and the firn interval velocity model for each iteration of the IRLS scheme. For $N$ snow pit measurements or runs of the extracted core, $V_{\text {core }}$ and $V_{\text {est }}$ represent the LMO, rms, or interval velocities of the measured and estimated data, respectively. The RMSE statistic of each iteration was compiled into the RMSE function (Figures D.3c - D.5c). I applied the rise over run formula (B.10) at a lag separation of one to estimate the RMSE gradient. Once the gradient of the RMSE function was less than $0.1 \%$, the velocity model had converged. The index $\tau$ was extracted from the first iteration that converged from the gradient of the RMSE function. A value for $\tau$ was mapped to each velocity interval for each iteration of both models. Because $\tau$ is a single statistic that describes an array of velocity possibilities at depth, I averaged all values of $\tau$ for the LMO and NMO horizons. This process represents a single simulation. I realized 1000 simulations to determine $\tau$. 

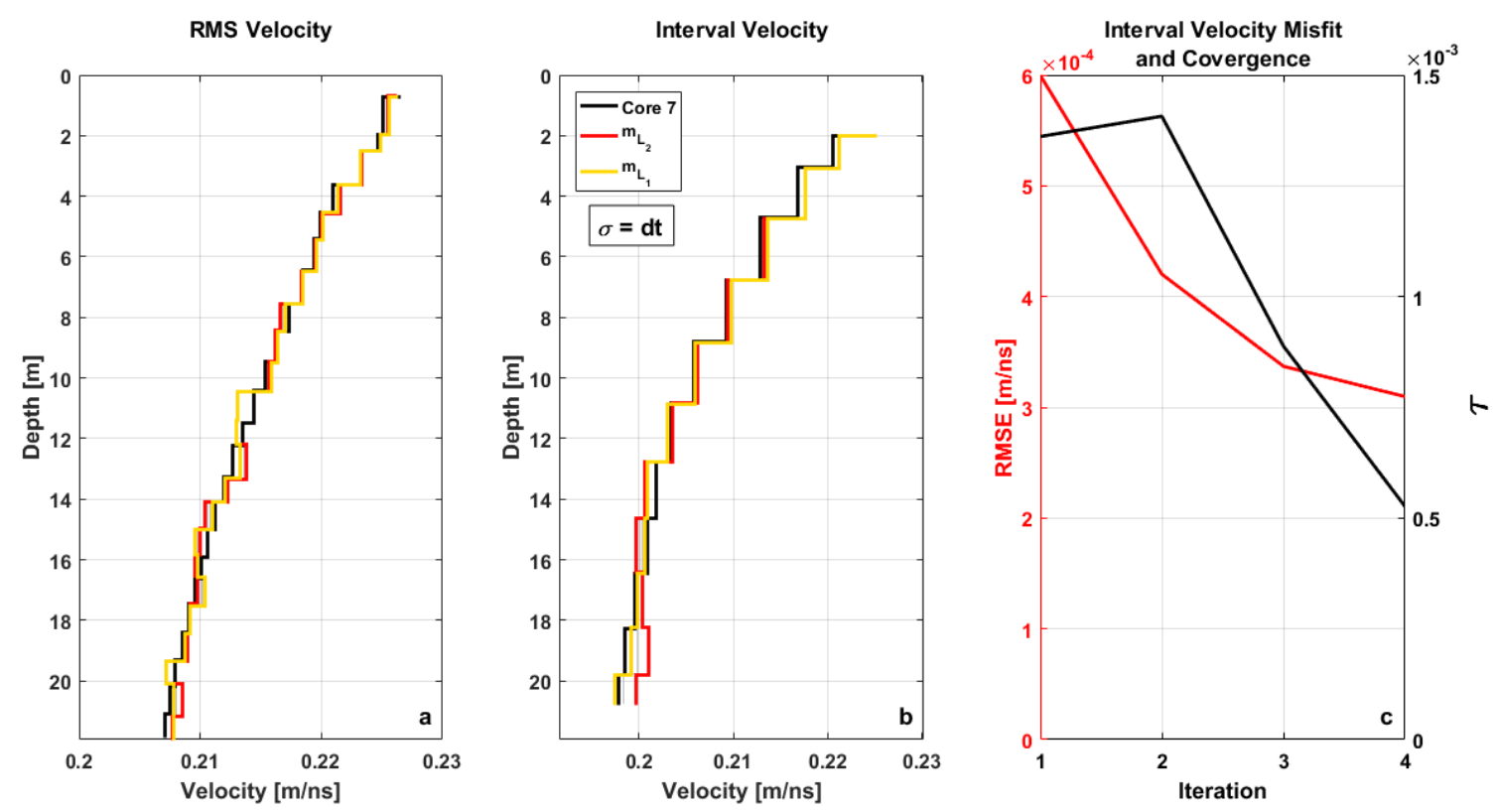

Figure D.3: The rms velocity solution for the synthetic travel-time model of Core Site 7 is produced in Panel a. Dix's solution for the interval velocity model is represented in Panel b. The number of intervals has been decimated by a factor of two to stabilize the solution. The RMSE function and $\tau$ factor are presented in Panel c. for each iteration. Random noise was added from the normal distribution of $\mu=0$ and $\sigma=\mathrm{dt}$. 

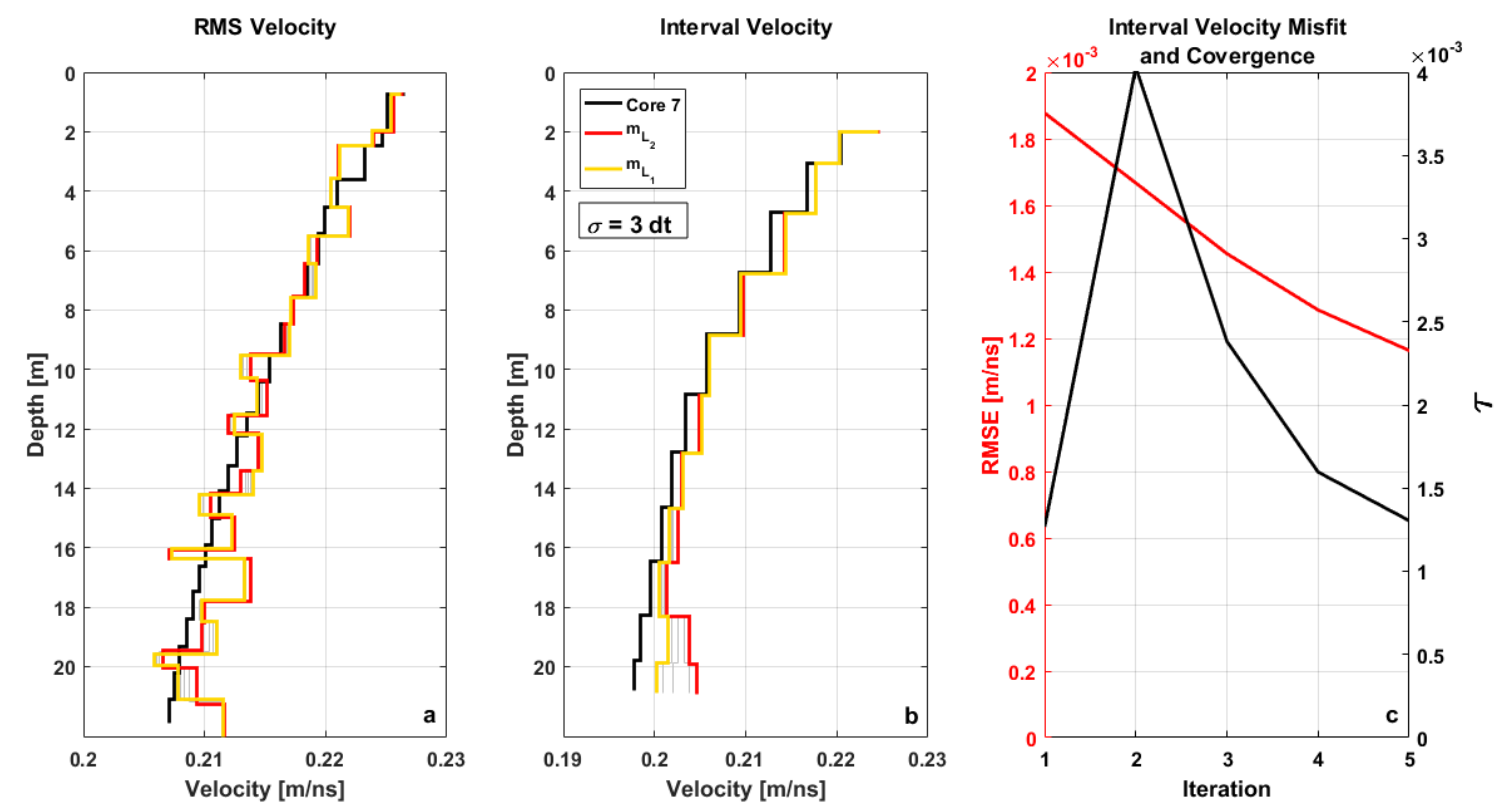

Figure D.4: Random noise was added from the distribution of $\sigma=3 \mathrm{dt}$ for this randomly drawn simulation. The value $\tau$ is selected after iteration 5. However, a very similar convergence factor is produced after the first iteration. In practice this solution would have converged after a single iteration, though the RMSE gradient is still decreasing. This represents a drawback to the global choice for $\tau$. 

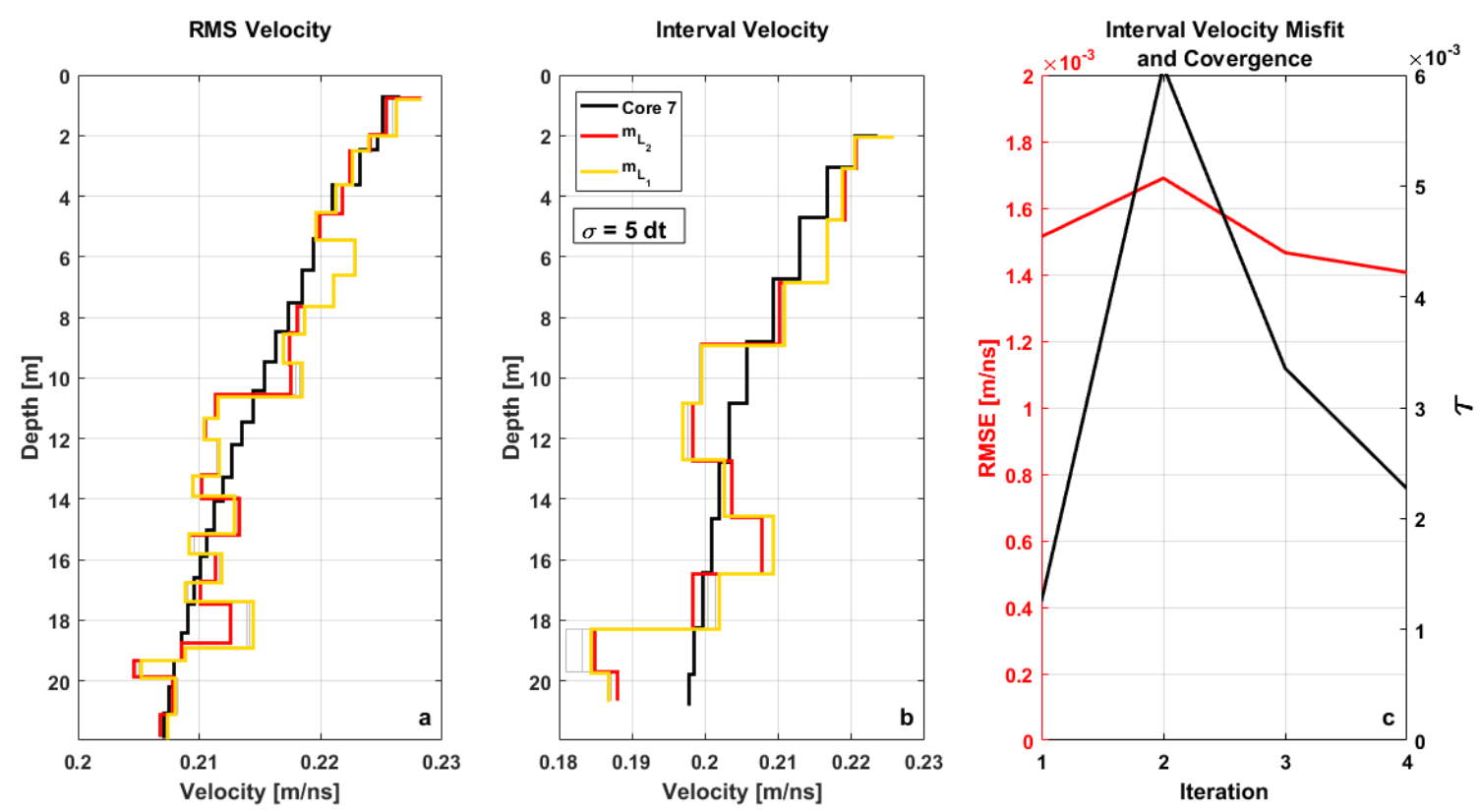

Figure D.5: The high noise level selected randomly from a Gaussian distribution of $\sigma=5 \mathrm{dt}$ produces large errors in the recovered rms and interval velocity models. Convergence generally occurs at a higher value $\tau$ in higher noise regimes. Here, the RMSE of the interval velocity profile is not greatly improved by the $\mathrm{L}_{1}$ solution. 


\section{D.5 Critically Refracted Lateral Waves}

Lateral energy travels on direct direct rays paths from transmitter to receiver, but also on critically refracted wave paths at the free-surface. An upgoing reflected wave can become critically refracted along the air/snow interface upon exiting the snow surface. These lateral wavepaths are identified in Figure 3.2. A snow pack model with this critically refracted raypath is sketched in Figure D.6. The following exercise calculates the travel-time of the wave following the hypothesized path.

\section{Critical Refraction Raypath for Homogeneous Snow Model}

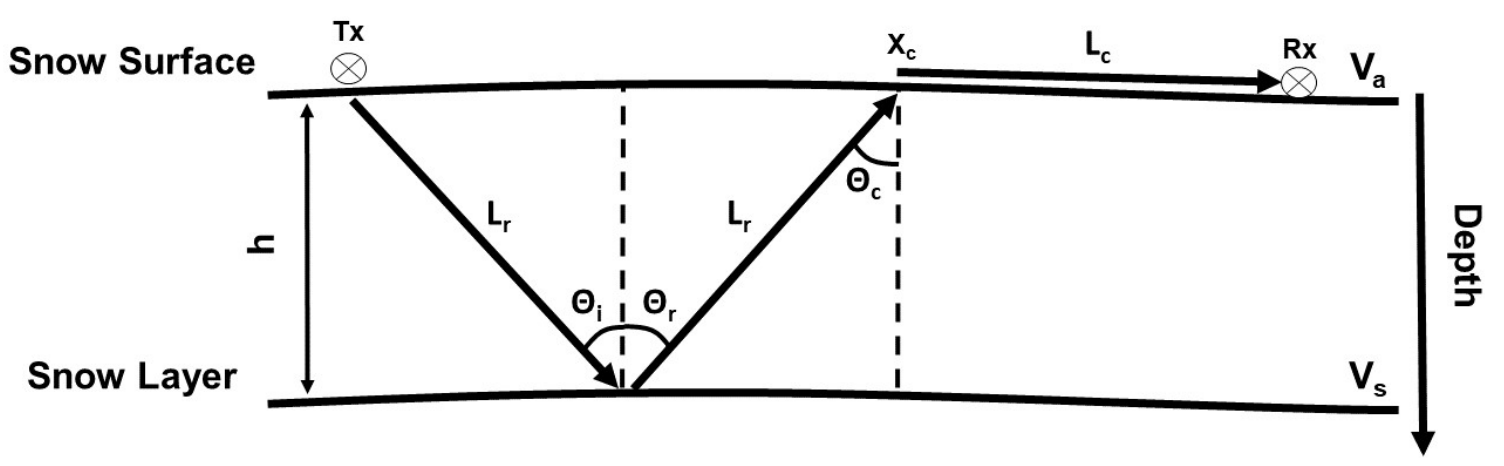

Figure D.6: The raypath of a critically refracted wave traveling through a homogeneous snow pack model. The wave is reflected at a layer boundary of the snow and is refracted upon exiting the snow surface.

The snow pack is homogeneous with a thickness $(h)$ and EM velocity $\left(V_{s}\right)$. A half-space of air $\left(V_{a}\right)$ is modeled above the snow surface. The transmitter and receiver antennas are on the snow surface and are separated by some offset $(X)$. In a homogeneous medium at a reflecting interface, the reflection angle $\left(\theta_{r}\right)$ is equal to the incidence angle $\left(\theta_{i}\right)$. Except for the case of total reflection, incoming radiation is also refracted (transmitted) at the layer interface. When a wave is traveling from a 
slower medium to a faster medium, according to Snell's Law

$$
\frac{\sin \theta_{1}}{\sin \theta_{2}}=\frac{V_{1}}{V_{2}}
$$

there is an angle of incidence that causes a critical refraction - known as the critical angle $\left(\theta_{c}\right)$. Critically refracted energy is refracted at $90^{\circ}$ and travels along the interface boundary within the faster medium. By setting $\theta_{2}=90^{\circ}, \theta_{c}$ is solved.

$$
\begin{aligned}
\frac{\sin \theta_{c}}{\sin 90} & =\frac{V_{1}}{V_{2}} \\
\theta_{c} & =\sin ^{-1}\left(\frac{V_{1}}{V_{2}}\right)
\end{aligned}
$$

In Figure D.6, $\theta_{i}=\theta_{r}=\theta_{c}$. A critical refraction arises along the free-surface boundary when this equality is satisfied. The critical distance $\left(X_{c}\right)$ can be solved, known $\theta_{c}$ and $h$.

$$
X_{c}=h \cdot \tan \theta_{c}
$$

The refraction path length

$$
L_{c}=X-X_{c}
$$

and the NMO reflection path length $2 L_{r}$, where

$$
L_{r}=\operatorname{sqrt}\left(\frac{X_{c}}{2}\right)^{2}+h^{2}
$$

are summed to calculate the lateral raypath length

$$
L=2 L_{r}+L_{c}
$$


The travel-time

$$
T_{c}=\frac{2 L_{r}}{V_{s}}+\frac{L_{c}}{V_{a}}
$$

from Tx to Rx is calculated for any offset beyond $X_{c}$.

Travel-times calculated from this model can be compared to the lateral waves identified in Figure 3.2. Residual travel-time corrections are not applied to Figure 3.2. Add these approximate travel-time corrections to the data while comparing the modeled travel-times: $(\mathbf{4} \mathbf{~ m}) \sim 1 n s,(\mathbf{8} \mathbf{~ m}) \sim 1.5$ ns, $(\mathbf{1 2} \mathbf{~ m}) \sim 3$ ns. For reference 0.5 wavelet cycles is $\sim 0.5 \mathrm{~ns}$.

The zone of lateral waves occupies the region bounded by the direct air-coupled arrival and the initial reflected arrival. Two model scenarios were tested to determine the potential refracted raypaths and travel-times for the antenna offsets $4 \mathrm{~m}, 8$," and $12 \mathrm{~m}$. The independent model parameters are the snow EM velocity $\left(V_{s}\right)$ and the snow layer thickness $(h)$. The critical angle $\left(\theta_{c}\right)$ and critical distance $\left(X_{c}\right)$ are dependent on the parameter choice. $V_{s}$ and $h$ were chosen after examining the Core 15 Western Spur Snow Pit density profile (Figure 3.6).

Model scenario (1): $V_{s}=0.2325 \mathrm{~m} / \mathrm{ns}$ and $h=0.75 \mathrm{~m}$. A fluctuation in the snow density at $0.75 \mathrm{~m}$ is plausibly responsible for a reflection at this depth. The velocity $\left(V_{s}\right)$ for model $(\mathbf{1})$ is the rms velocity calculated in $10 \mathrm{~cm}$ intervals to $0.75 \mathrm{~m}$ depth via equation (D.21), after density-velocity conversion via the CRIM equation (2.27). By equation (D.28), $\theta_{c} \sim 51^{\circ}$, and equation (D.29), $X_{c}=1.86 \mathrm{~m}$. The modeled traveltime for offsets $4 \mathrm{~m}, 8 \mathrm{~m}$, and $12 \mathrm{~m}$ are $\sim 17 n s, \sim 31 \mathrm{~ns}$, and $\sim 44 \mathrm{~ns}$, respectively. Refer back to Figure 3.2. On the $4 m$ offset gather, the refracted arrivals are identified in the zone bounded by the surface wave and reflected wave. At $8 m$ offset, faint but extant critically refracted energy is bounded by the air wave and the surface wave. 
On the $12 m$ offset gather, the lateral waves are identified in the zone bounded by the air wave and surface.

Model scenario (2): $V_{s}=0.2285 \mathrm{~m} / \mathrm{ns}$ and $h=1.80 \mathrm{~m}$. The velocity $\left(V_{s}\right)$ for model $(2)$ is the rms velocity calculated in $10 \mathrm{~cm}$ intervals extrapolated to $1.80 \mathrm{~m}$ depth via equation (D.21), after density-velocity conversion via the CRIM equation (2.27). The snow thickness $(h)$ is approximately the depth measured by the NMO velocity analysis at the snow pit location prior to depth bias correction of the annual horizon (Chapter 3.3.1). By equation (D.28), $\theta_{c} \sim 50^{\circ}$, and equation (D.29), $X_{c}=$ $4.27 \mathrm{~m}$. A critically refracted arrival is not observed at $4 \mathrm{~m}$, because the critical distance is greater than the antenna offset. For offsets $8 \mathrm{~m}$ and $12 \mathrm{~m}$ the modeled travel-times are $\sim 37$ and $\sim 50 \mathrm{~ns}$, respectively. On the $8 \mathrm{~m}$ offset gather the refracted waves arrive between the surface wave and reflected wave. Within this zone a reduction in lateral wave cycles is caused by the interference of the direct, refracted, and reflected waves. At $12 \mathrm{~m}$ offset the faint refracted amplitudes arrive ahead of the direct surface wave.

This exercise was intended to define the lateral wave phenomena with a simplistic approach. The model parameters were constrained by in situ observations. The results of the model validate the hypothesis of critically refracted arrivals. The wavefield features important to the velocity analysis have been identified in Figure 3.2 and are discussed in Chapter 2.4. 